\title{
RECEIVED
}

JAN 291997

OSTI

OAK RIDGE NATIONAL LABORATORY

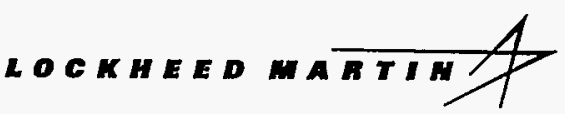

\section{Characterization of the MVST Waste Tanks Located at ORNL}

J. M. Keller

J. M. Giaquinto

A. M. Meeks
MANAGED AND OPERATED BY

LOCKHEED MARTN ENERGY RESEARCH CORPORATION FOR THE UNTED STATES DEPARTMENT OF ENERGY

ORNL-27 (3-96) 
This report has been reproduced directly from the best available copy.

Available to DOE and DOE contractors from the Office of Scientific and Technical Information, P. O. Box 62, Oak Ridge, TN 37831; prices available from (423) 576-8401, FTS 626-8401.

Available to the public from the National Technical Information Service, U.S. Department of Commerce, 5285 Port Royal Road, Springfield. VA 22161.

This report was prepared as an account of work sponsored by an agency of the United States Government. Neither the United States Government nor any agency thereof, nor any of their employees, makes any warranty, express or implied, or assumes any legal liability or responsibility for the accuracy, completeness, or usefulness of any information, apparatus, product, or process disclosed, or represents that its use would not infringe privately owned rights. Reference herein to any specific commercial product, process, or service by trade name. trademark, manufacturer, or otherwise, does not necessarily constitute or imply its endorsement, recommendation, or favoring by the United States Government or any agency thereof. The views and opinions of authors expressed herein do not necessarily state or reflect those of the United States Government of any agency thereof. 


\title{
Characterization of the MVST Waste Tanks Located at ORNL
}

\author{
J. M. Keller
}

J. M. Giaquinto

A. M. Meeks

December 1996

\author{
Prepared by the \\ OAK RIDGE NATIONAL LABORATORY \\ Oak Ridge, Tennessee 37831 \\ managed by \\ LOCKHEED MARTIN ENERGY RESEARCH CORP. \\ for the \\ U.S. DEPARTMENT OF ENERGY \\ under contract \\ DE-AC05-96OR22464
}




\section{DISCLAIMER}

This report was prepared as an account of work sponsored by an agency of the United States Government. Neither the United States Government nor any agency thereof, nor any of their employees, make any warranty, express or implied, or assumes any legal liability or responsibility for the accuracy, completeness, or usefulness of any information, apparatus, product, or process disclosed, or represents that its use would not infringe privately owned rights. Reference herein to any specific commercial product, process, or service by trade name, trademark, manufacturer, or otherwise does not necessarily constitute or imply its endorsement, recommendation, or favoring by the United States Government or any agency thereof. The views and opinions of authors expressed herein do not necessarily state or reflect those of the United States Government or any agency thereof. 


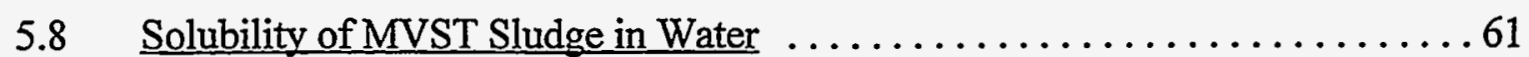

5.9 Estimates for Compliance with WIPP WAC, Rev. 5 for MVST Sludge $\ldots \ldots .62$

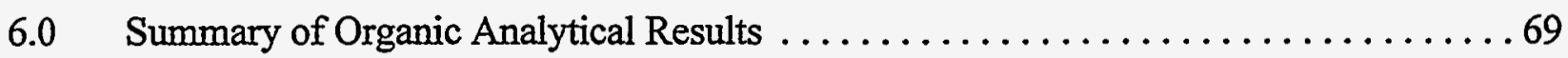

6.1 Discussion of Organic Analysis . . . . . . . . . . . . . . . . . . . 75

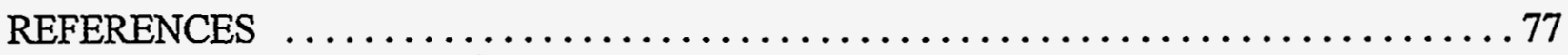

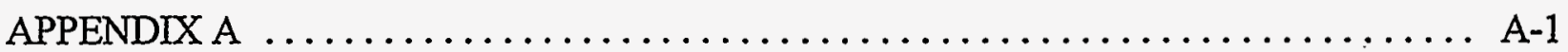

QC Acceptance Criteria for Radioactive Liquid/Solid Waste Samples .......... A-1

Volatile Organic Analyses QC Limits .................... A-4

Nonhalogenated Volatile Organic Analyses QC Limits ............ A-5

Semivolatile Organic Analyses QC Limits .................. A-5

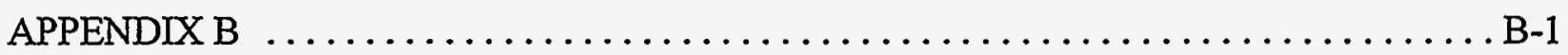

Table B1 Total Mass and Activity for Selected Species of Interest in Sludge ..... B-2

Table B2 Dose Measurements on Liquid Samples . .................. B-3

Table B3 Dose Measurements on Sludge Samples .................. B-3 


\section{LIST OF TABLES}

Table 1 Summary of Tanks in the Active ORNL LLLW System $\ldots \ldots \ldots \ldots \ldots \ldots 2$

Table 2

Table 3

Table 4

Table 5

Table 6

Table 7

Table 8

Table 9

Table 10

Table 11

Table 12

Table 13

Table 14

Table 15

Table 16

Table 17

Table 18

Table 19

Table 20

Table 21

Table 22

Table 23

Table 24

Table 25
Summary of Tanks in the Active ORNL LLLW System
Volumes Estimates for Liquid and Sludge in the LLLW System $\ldots \ldots \ldots \ldots . .3$

Analytical Data for Liquids in Tanks W-24, W-25, and W-26 ..........23

Analytical Data for Liquids in Tanks W-27, W-28, and W-31 $\ldots \ldots \ldots \ldots . .27$

Analytical Data for Sludge in Tanks W-24, W-25, and W-26 ........... 31

Analytical Data for Sludge in Tanks W-27, W-28, and W-31 ......... 35

Summary of Quality Checks for MVST Supernatant Data ............41

Distribution of Beta Activity in Supernatant ................... 43

Assumption Used for Major Compounds in MVST Sludge $\ldots \ldots \ldots \ldots \ldots 45$

Summary of Quality Checks for MVST Sludge Data $\ldots \ldots \ldots \ldots \ldots \ldots . \ldots 45$

Distribution of Beta Activity in MVST Sludge .................. 48

Summary of Actinide Elements in MVST Sludge ................ 49

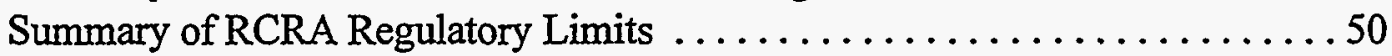

Summary of Denature Ratios for MVST Supernatant ............... 53

Summary of Denature Ratios for MVST Sludge ................. 53

Example of Converting Atom \% to Weight \% for W-31 Sludge .......... 54

Summary of Total Anion Data for MVST Sludge ................. 57

Recovery of Selected Species in Water Leach ...................61 61

Estimates for ${ }^{239} \mathrm{Pu}$ FGE with the MVST Sludge ................6. 63

Estimates for ${ }^{239} \mathrm{Pu}$ Equivalent Activity with the MVST Sludge . . . . . . . 64

Isotopes that Contribute to the Decay Heat in the MVST Sludge ..........65

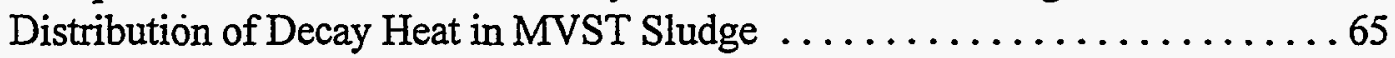

Summary of Relative Decay Heat in MVST Sludge ................66

Analytical Organic Data for MVST Liquid Samples ................ 71

Analytical Organic Data for MVST Sludge Samples .............. 73 


\section{LIST OF FIGURES}

Figure 1 Distribution of Major Cations and Anions in Liquid Phase $\ldots \ldots \ldots \ldots \ldots . . \ldots 40$

Figure 2 Distribution of Selected Ionic Species in Liquid Phase . . . . . . . . . . . . 40

Figure 3 Distribution of Major Compounds in MVST Sludge $\ldots \ldots \ldots \ldots \ldots \ldots 47$

Figure 4 Distribution of Uranium and Thorium in MVST Sludge .............. 47

Figure 5 Distribution of Plutonium by Alpha Activity in MVST Sludge $\ldots \ldots \ldots \ldots . .55$

Figure 6 Distribution of Plutonium by Concentration in MVST Sludge ........... 55

Figure 7 Distribution of Beta Decay Heat in MVST Sludge $\ldots \ldots \ldots \ldots \ldots \ldots \ldots 67$

Figure 8 Distribution of Alpha Decay Heat in MVST Sludge ................67 67 


\section{ACKNOWLEDGMENTS}

The authors wish to express appreciation to the following staff members of the ORNL Chemical and Analytical Sciences Division who made important contributions to this work:

Inorganic and Radiochemical Analytical Support

L. D. Bible

R. D. Canaan

D. A. Caquelin

K. M. Hoyle

C. L. Kay ${ }^{\mathrm{a}}$

E. H. McBay

T. P. Mills ${ }^{\mathrm{a}}$

D. H. Smith

N. A. Teasley

Organic Analytical Support

S. H. Harmon

R. L. Schenley

L. T. Worthy ${ }^{\mathrm{b}}$

a Subcontractor staff, Oak Ridge Research Institute.

${ }^{b}$ Subcontractor staff, Midwest Technical Inc.

The authors also wish to express appreciation to the following staff members of the Liquid and Gaseous Waste Operation Department of the Office of Waste Management and Remedial Actions for tank sampling support:

Sampling Radioactive Waste Tanks

J. D. Brewer

C. B. Scott 


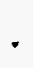




\section{ABBREVIATIONS AND ACRONYMS}

$\begin{array}{ll}\text { ALARA } & \text { As Low As Reasonably Achievable } \\ \text { CAO } & \text { Carlsbad Area Office } \\ \text { CASD } & \text { Chemical and Analytical Sciences Division } \\ \text { CVAA } & \text { Cold Vapor Atomic Absorption } \\ \text { DOT } & \text { Department of Transportation } \\ \text { DQO } & \text { Data Quality Objective } \\ \text { EPA } & \text { Environmental Protection Agency } \\ \text { GC/MS } & \text { Gas Chromatography/Mass Spectrometry } \\ \text { GC } & \text { Gas Chromatography } \\ \text { GFAA } & \text { Graphite Furnace Atomic Absorption } \\ \text { IC } & \text { Ion Chromatography } \\ \text { ICP } & \text { Inductively Coupled Plasma } \\ \text { ICP-AES } & \text { Inductively Coupled Plasma - Atomic Emission Spectroscopy } \\ \text { ICP-MS } & \text { Inductively Coupled Plasma - Mass Spectrometry } \\ \text { IDL } & \text { Instrument Detection Limit } \\ \text { LCS } & \text { Laboratory Control Sample } \\ \text { LLLW } & \text { Liquid Low-Level Waste } \\ \text { LMER } & \text { Lockheed Martin Energy Research, Corp. } \\ \text { LMES } & \text { Lockheed Martin Energy Systems, Inc. } \\ \text { MDL } & \text { Method Detection Limit } \\ \text { MS } & \text { Matrix Spike } \\ \text { MSD } & \text { Matrix Spike Duplicate } \\ \text { MVST } & \text { Melton Valley Storage Tanks } \\ \text { NHVOA } & \text { Non-halogenated Volatile Organic Analysis } \\ \text { NTS } & \text { Nevada Test Site } \\ \text { ORNL } & \text { Oak Ridge National Laboratory } \\ \text { PCB } & \text { Polychlorinated Biphenyls } \\ \text { QA } & \text { Quality Assurance } \\ \text { QAPjP } & \text { Quality Assurance Project Plan } \\ \text { QAPP } & \text { Quality Assurance Program Plan } \\ \text { QC } & \text { Quality Control } \\ \text { RCRA } & \text { Resource Conservation and Recovery Act } \\ \text { RMAL } & \text { Radioactive Materials Analytical Laboratory (Building 2026) } \\ \text { SVOA } & \text { Semivolatile Organic Analysis } \\ \text { TC } & \text { Total Carbon } \\ \text { TCLP } & \text { Toxicity Characteristic Leaching Procedure } \\ \text { TDS } & \text { Total Dissolved Solids } \\ \text { TIC } & \text { Total Inorganic Carbon or Tentatively Identified Compounds } \\ \text { TIMS } & \text { Thermal Ionization Mass Spectrometry } \\ \text { TOC } & \text { Total Organic Carbon } \\ \text { TRU } & \text { Transuranic } \\ \text { TWCP } & \text { Transuranic Waste Characterization Program } \\ \text { VOA } & \text { Volatile Organic Analysis } \\ \text { WAC } & \text { Waste Acceptance Criteria } \\ \text { WIPP } & \text { Waste Isolation Pilot Plant } \\ & \end{array}$




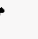




\section{EXECUTIVE SUMMARY}

During the fall of 1996 there was a major effort to sample and analyze the Active Liquid Low-Level Waste (LLLW) tanks at ORNL which include the Melton Valley Storage Tanks (MVST) and the Bethel Valley Evaporator Service Tanks (BVEST). The characterization data summarized in this report was needed to address waste processing options, address concerns of the performance assessment (PA) data for the Waste Isolation Pilot Plant (WIPP), evaluate the waste characteristics with respect to the waste acceptance criteria (WAC) for WIPP and Nevada Test Site (NTS), address criticality concerns, and meet DOT requirements for transporting the waste. This report only discusses the analytical characterization data for the MVST waste tanks (except for W-29 and W30). There will be a companion report on the BVEST waste tanks that will include the analytical data and the results from rheometry experiments on the BVEST sludge.

The isotopic data presented in this report supports the position that fissile isotopes of uranium ${ }^{233} \mathrm{U}$ and ${ }^{235} \mathrm{U}$ ) and plutonium $\left({ }^{239} \mathrm{Pu}\right.$ and $\left.{ }^{241} \mathrm{Pu}\right)$ were "denatured" as required by the administrative controls stated in the ORNL LLLW waste acceptance criteria (WAC). In general, the MVST sludge was found to be both hazardous by RCRA characteristics and the transuranic alpha activity was well above the $100 \mathrm{nCi} / \mathrm{g}$ limit for TRU waste. The characteristics of the MVST sludge relative to the WIPP WAC limits for fissile gram equivalent, plutonium equivalent activity, and thermal power from decay heat, were estimated from the data in this report and found to be far below the upper boundary for any of the remote-handled transuranic waste (RH-TRU) requirements for disposal of the waste in WIPP. 


\title{
Characterization of the MVST Waste Tanks Located at ORNL
}

\author{
J. M. Keller, J. M. Giaquinto, A. M. Meeks
}

\subsection{Introduction}

The active ORNL Liquid Low Level Waste (LLLW) system consists of the set of waste tanks summarized in Table 1. As indicated in Table 1, this report only discusses the analytical characterization data for the MVST waste tanks (except for W-29 and W-30). There will be a companion report on the BVEST waste tanks in the near future. The characterization data summarized in this report was needed to address waste processing options, address concerns of the performance assessment (PA) for the Waste Isolation Pilot Plant (WIPP), evaluate the waste characteristics with respect to the waste acceptance criteria (WAC) for WIPP and Nevada Test Site (NTS), address criticality concerns, and to meet DOT requirements for transporting the waste.

The data was collected during a sampling and analysis campaign performed during the late summer and fall of 1996. The sampling and waste characterization requirements were documented in a Sampling and Analysis Plan ${ }^{1}$ (SAP). The level of quality assurance approximates that required for regulatory measurements with the understanding that, when needed, sample size requirements were reduced, and steps were taken to reduce sample handling to ensure radiation exposures were aslow-as-reasonably-achievable (ALARA). Some procedure modifications were required to handle chemical matrix problems due to the high levels of sodium nitrate, uranium, and thorium present. Any deviations from procedures or problems observed with the tank samples were documented in the data files maintained by the laboratory. The regulatory holding time requirements for mercury and the organic measurements were complied with unless noted differently in the data tables. The Quality Control (QC) Acceptance Criteria for measurement used on this project are summarized in Appendix A. 
Table 1 Summary of Tanks in the Active ORNL LLLW System

\begin{tabular}{|c|c|c|}
\hline \multirow{2}{*}{ Tanks } & \multicolumn{2}{|c|}{ Data Presented in this report } \\
\hline & Liquid & Sludge \\
\hline \multicolumn{3}{|c|}{ BVESI IANKS } \\
\hline $\mathrm{C}-1$ (HLW) & none & none \\
\hline $\mathrm{C}-2(\mathrm{HLW})$ & none & none \\
\hline W-21 (PWTP) & none & none \\
\hline W-22 (BVCT) & none & none \\
\hline W-23 (LLLW) & none & none \\
\hline \multicolumn{3}{|c|}{ 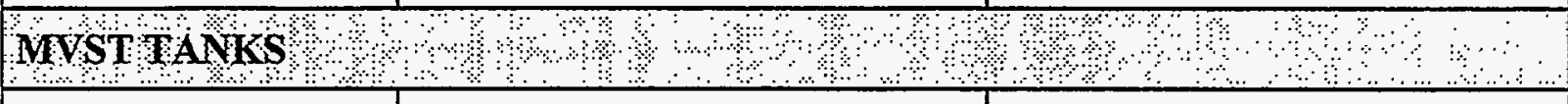 } \\
\hline W-24 & $\checkmark$ & $\checkmark$ \\
\hline $\mathrm{W}-25$ & $\checkmark$ & $\checkmark$ \\
\hline W-26 & $\checkmark$ & $\checkmark$ \\
\hline W-27 & $\checkmark$ & $\checkmark$ \\
\hline W-28 & $\checkmark$ & $\checkmark$ \\
\hline W-29 & none & none \\
\hline W-30 & none & none \\
\hline W-31 & $\checkmark$ & $\checkmark$ \\
\hline
\end{tabular}

The earlier waste tank characterization work performed, in 1985 by Peretz ${ }^{2}$ et. al. and 1990 by Sears $^{3}$ et. al., did not specifically address criticality concerns. There was limited radiochemical data on ${ }^{233} \mathrm{U},{ }^{235} \mathrm{U}$ and ${ }^{239} \mathrm{Pu}$; which was taken from gross radiochemical screening measurements. This previous data for fissile actinide elements in the LLLW waste tanks had relatively large analytical errors and should be used with caution. More recent data, reported by $\mathrm{Keller}^{5}$ et.al. and Sears ${ }^{6}$, which was collected in early 1996, addresses some of the criticality concerns but did not address all the tanks of interest. The analytical data for fissile isotopes in this report are based on mass spectrometry measurements, similar to the data collected in early 1996, but includes a more complete set of LLLW waste tanks. The uranium and plutonium were each chemically separated from the 
waste matrix prior to measurement of the isotopic ratios by mass spectrometry. The mass spectrometry measurements yield more detailed and accurate information than radiochemical measurements for the major fissile isotopes present. The isotopic mass ratio measurements on the sludge samples may not represent the average isotopic ratios due to the heterogeneous nature of the sludge. The isotopic data for each liquid sample should be more representative of the overall supernatant present than comparable measurements for the sludge. Based upon physical observations, the tank sludge tends to be segregated into vertical layers which indicates minimal mixing of the sludge material as it was added to the tanks. Due to limited access to the waste tanks, there is no analytical data available to evaluate segregation horizontally across the tank at the time of this report.

An inventory of radioactive liquid waste and sludge stored in each tank are shown in Table 2 and includes estimates for the volumes through October 1996. The volume data ${ }^{4}$ is based on estimates by the Chemical Technology Division (CTD).

Table 2 Volumes Estimates for Liquid and Sludge in the LLLW System

\begin{tabular}{|c|c|c|c|c|c|c|}
\hline \multirow{2}{*}{ Tank } & \multicolumn{2}{|c|}{ Total Waste Volume } & \multicolumn{2}{|c|}{ Sludge Volume } & \multicolumn{2}{|c|}{ Supernatant Volume } \\
\hline & (gal) & (L) & (gal) & (L) & (gal) & $(\mathrm{L})$ \\
\hline W-21 & 23100 & 87500 & 6500 & 24600 & 16600 & 62900 \\
\hline W-22 & 13100 & 49600 & 6800 & 25800 & 6300 & 23800 \\
\hline W-23 & 21800 & 82600 & 10600 & 40100 & 11200 & 42400 \\
\hline W-24 & 22300 & 84400 & 8700 & 32900 & 13600 & 51500 \\
\hline W-25 & 44100 & 167000 & 17300 & 65500 & 26800 & 101500 \\
\hline W-26 & 44600 & 168900 & 11800 & 44700 & 32800 & 124200 \\
\hline W-27 & 26000 & 98500 & 16000 & 60600 & 10000 & 37900 \\
\hline W-28 & 44200 & 167400 & 4500 & 17000 & 39700 & 150300 \\
\hline W-29 & 44300 & 167800 & $11000^{\circ}$ & 41700 & 33300 & 126100 \\
\hline $\mathrm{W}-30$ & 41200 & 156000 & 11000 & 41700 & 30200 & 114300 \\
\hline W-31 & 43900 & 166200 & 10600 & 40100 & 33300 & 126100 \\
\hline
\end{tabular}




\subsection{Sample Collection Activities}

A detailed description on the background, operation of the LLLW system, and the sample collection techniques has been presented in previous reports and will not be discussed here (see Sections 2 and 3 of Reference 3). The staff from the Liquid and Gaseous Waste Operations (LGWO) provided all sample collection support and delivered the samples to the analytical laboratory. A good description of the sampling procedures is provided in Appendix A of the Sampling and Analysis Plan ${ }^{1}$; a current copy of these procedures are available from the LGWO group. The documentation for chain-of-custody was prepared, maintained for each sample collected, and stored with the data files by the analytical laboratory.

\subsection{Analytical Methodology}

The information and data collected from these studies are used to support various activities. The activities include demonstration of regulatory compliance, measurements to support future processing options, and to meet data needs for risk assessments and other safety related assessments such as criticality. Standardized analytical procedures are used to the extent possible to ensure broad acceptance of the data generated. Unless stated otherwise, the U. S. Environmental Protection Agency (EPA) methods are used for the analyses of constituents listed as hazardous under the Resource Conservation and Recovery Act (RCRA), which includes all the inorganic and organic measurements presented in this report: In general the EPA Guidance Manual, Test Methods for Evaluating Solid Waste ${ }^{7}(S W-846)$, is used for inorganic and organic methods. Some modifications of the standard procedures are necessary to handle the high radiation levels and the high salt/solids content. Some procedure modifications are required to generate valid data; these changes were usually needed to correct for chemical or other matrix related interferences. All deviations from the standard procedures are documented in the raw data files and can be provided upon request to data users. 


\subsection{Sample Preparation}

The aqueous supernatant samples from the waste tanks were filtered or centrifuged to remove suspended particles. The clarified liquids were then digested by the SW-846 Method 3015, Microwave Assisted Acid Digestion of Aqueous Samples and Extracts. This sample preparation for aqueous samples was then used for all subsequent metal analyses by ICP-AES and GFAA, and most of the radiochemical analyses. Based upon results from a collaborative study ${ }^{8}$ with Argonne National Laboratory -- East (ANL-E), Method 3015/3051 demonstrated excellent recovery for mercury and was used to prepare tank samples for mercury determination.

The primary method for digesting the sludge samples was SW-846 Method 3051, Microwave Assisted Acid Digestion of Sediments, Sludges, Soils, and Oils. This sample preparation is considered to be a total digestion for metals and radionuclides by regulatory agencies and yields good results for most metals and radionuclides of interest. This digestion gave poor performance on two of the metals of interest, silver and silicon. Although nitric acid is excellent for dissolving silver compounds, there is usually enough chloride present in waste samples to form an insoluble silver chloride $(\mathrm{AgCl})$ precipitate. If the chloride concentration is increased sufficiently, a silver chloride complex $\left(\mathrm{AgCl}_{3}^{-2}\right)$ forms which is soluble in the aqueous environment. Improved matrix spike recovery and defensible data for silver were obtained using a separate sample digestion discussed later in this report.

If the total silicon content in the sludge must be known to develop waste treatment options such as vitrification, another sample digestion is required. A simple nitric acid treatment will not dissolve most siliceous materials. The SW-846 Method 3052, Microwave Assisted Acid Digestion of Siliceous and Organically Based Matrices, provides the necessary digestion chemistry to yield good silicon data. Sludge samples were prepared for measurement of total silicon, by taking approximately $0.5 \mathrm{~g}$ of sludge and mixing with $7 \mathrm{~mL}$ of concentrated nitric acid and $3 \mathrm{~mL}$ of hydrofluoric acid in a fluorocarbon microwave vessel. The samples were digested for 10 minutes at $95 \%$ full power ( 570 watts) and then cooled to room temperature. The acid solution was then treated with excess boric acid and heated to $80^{\circ} \mathrm{C}$ for ten minutes to complex any free fluoride. This digestion mixture is cooled, filtered into a $50 \mathrm{~mL}$ volumetric flask, and diluted to volume with 
ASTM Type II water. Care must be exercised to ensure the digestion solution is cooled to room temperature prior to opening the sealed microwave vessel or there may be a significant loss of the volatile $\mathrm{SiF}_{4}$. The free fluoride is complexed with the boron to protect the sample introduction system to the ICP-AES and to prevent a high silicon background from the instrument glassware. This sample digestion with hydrofluoric acid should not be used for radiochemical measurements, especially for measurement of lanthanides or actinides.

Most of the metal and radionuclide data presented in this report are based upon a Method 3051 digestion with approximately a 0.5 gram sludge sample and $10 \mathrm{~mL}$ of concentrated nitric acid. After the microwave digestion is completed and the solution cooled to room temperature, the sample is filtered into a volumetric flask and diluted to $50 \mathrm{~mL}$ with ASTM Type II water or better. To ensure valid silver and antimony data, samples were digested in a similar manner except the $10 \mathrm{~mL}$ of nitric acid was replaced with $6 \mathrm{~mL}$ of concentrated nitric acid plus $4 \mathrm{~mL}$ of concentrated hydrochloric acid. Any residue remaining after the nitric acid or nitric-hydrochloric acid digestion consisted of mostly $\mathrm{SiO}_{2}$ and was discarded.

\subsection{Metal Analysis}

Three analytical measurement methods were used to determine all of the metals included in this report. Most of the metals are first determined by SW-846 Method 6010A, Inductively Coupled Plasma - Atomic Emission Spectroscopy (ICP-AES). There are several elements of interest for which the ICP-AES has insufficient detection limits, and these elements must be determined by Method 7000A, Atomic Absorption Methods. The Radioactive Materials Analytical Laboratory (RMAL) uses

a Graphite Furnace Atomic Absorption (GFAA) Spectrometer for elements that require better sensitivity. The elements that usually require GFAA were antimony (Method 7041), arsenic (Method 7060A), lead (Method 7421), selenium (Method 7740), and thallium (Method 7841). All the mercury measurements are done by either Method 7470A, Mercury in Liquid Waste (Manual ColdVapor Technique), or Method 7471A, Mercury in Solid or Semisolid Waste (Manual Cold-Vapor Technique). The samples discussed in this report were prepared for mercury analysis by the microwave technique discussed in section 3.1, the sample preparation specified in the mercury methods (7470A and 7471A) were not used. 
The level of radioactivity in most LLLW tank samples required that the analytical systems used for metal measurements be modified for operation in a radiochemical hood or glove box. Custom instrument configurations are necessary to ensure contamination control and worker safety. All work was performed in radiochemical laboratories which are operated under strict radiation protection programs, with the use of protective clothing and routine contamination monitoring. Both an ICP-AES system and a GFAA system can generate dry, dusty particles which are difficult to contain and are highly hazardous when radioactive. A detailed description of the RMAL setup for these instruments are given in Appendix B of Reference 3.

The instrument detection limits (IDL) for various metals with undiluted aqueous samples are listed in data tables along with the results. For sludge samples, these detection limits must be increased by a factor that represents the dilution that results from the sample preparation. For all the MVST sludge samples approximately $0.5 \mathrm{~g}$ of sample was digested and then diluted to $50 \mathrm{~mL}$ which results in about a 100 fold dilution for the sample, and thus a 100 fold increase in the detection limits.

The analytical error for the metal measurements depends upon the analytical method, the concentration level, and the chemical matrix. Inductively-coupled plasma-atomic emission spectroscopy (ICP-AES) and inductively coupled plasma-mass spectrometry (ICP-MS) are both multi-element measurement techniques that are designed for the best average performance for all elements analyzed. In general, these measurement techniques are not optimized for any single element. The sample introduction system for ICP instruments adds additional variability due to changes in sample density, viscosity, and solids content between samples and/or calibration standards. Overall, the expected analytical error for ICP measurements range from $\pm 4-6 \%$ at concentrations above 10 times the detection limit to $\pm 20-50 \%$ near the detection limit. These error estimates are typical for both ICP-AES and ICP-MS measurements.

Graphite Furnace AA instruments are generally optimized for a specific element and usually provide lower detection limits and better precision. The expected analytical error for GFAA measurements range from 3-5\% for concentrations greater than 10 times the detection limit to $20-40 \%$ near the detection limit. One advantage of GFAA analysis is that the measurements are normally well above the method's detection limits. The mercury measurements were done by Cold Vapor Atomic 
Absorption (CVAA), which is very selective and sensitive for mercury. The analytical errors for CVAA measurements are similar to GFAA work.

\subsection{Anion Analysis}

The determination of the inorganic anions was needed for the development of process treatment options, to provide information to explain the distribution and chemical behaviors observed in the waste tanks, and to ensure the major chemical constituents were identified in the waste for which data was used to calculate the mass and charge balance for each sample. The common inorganic anions; including fluoride, chloride, bromide, phosphate, nitrate, nitrite, and sulfate; were measured by ion chromatography (IC) with a Dionex Model 4500i system. In addition, several water soluble organic acids were measured along with the inorganic anions. These organic acids were measured in their ionized form and included formate, acetate, citrate, and oxalate. Both the citrate and the oxalate can form strong complexes with many metals and change the solution chemistry of these metals in the waste. The ion chromatography system used for measurements on these radioactive samples was configured such that the components that come into contact with radioactivity were isolated in a radiochemical hood for contamination control.

From past observations, the nitrate content dominates both the mass and charge balance calculations with both the supernatant and sludge samples taken from the active LLLW tanks. There are many other anions present in the waste, some of which are measured directly by ion chromatography and others which can be estimated from the metal data such as chromate, dichromate, permanganate, and others. The carbonate is estimated from the total inorganic carbon measurement.

The liquid samples were always analyzed directly by ion chromatography after an appropriate dilution with water. Accounting for the mass and charge balance with the aqueous samples requires less assumptions about the solution chemistry compared to the precipitation chemistry for the sludge samples. The mass/charge balance checks for aqueous samples should agree within the analytical error (approximately $\pm 10 \%$ ) of the measurements. The performance of balance checks for sludge samples is not expected to be as good as the liquid samples because of the large content 
of mixed oxides, hydrated hydroxides (heavy metals and actinides), and insoluble carbonates (calcium carbonate, etc.) present in the sludge. The complex precipitation chemistry of the sludge complicates the measurements of total anions and makes estimates for the mass and charge balance more difficult. Analytical techniques such as $\mathrm{x}$-ray fluorescence $(\mathrm{XRF})$ are useful for solid samples but are limited to total element measurements (total sulfur vs. sulfate, total phosphorus vs. phosphate). Another technique, $\mathrm{x}$-ray diffraction $(\mathrm{XRD})$, is useful for the determination of compounds present but only provides qualitative information such as the identification of crystal structures. For this report, the primary sludge anion data is based on a water leach which represents the sum of the anions in the interstitial liquid and the water soluble anions from the solids. For these measurements the sludge samples were prepared by adding approximately 1 gram of sludge to 10 $\mathrm{mL}$ of water, mixing for several minutes at room temperature on a vortex mixer, and separating the solids. The resulting solution was analyzed by ion chromatography and the anion concentration was normalized back to the wet weight of the sludge.

Based on conversations with chemists from the Savannah River Site (SRS) and the Hanford site, who have been involved with similar waste characterization work and the experience over the past five years by the RMAL laboratory, the water leach preparation of the caustic sludge samples provides the best total anion data for the halides, nitrites, nitrates, and fair data for sulfate. To resolve questions concerning the total anion content of the sludge two additional sample preparation methods were tested on the MVST sludge samples. The two preparation methods used were 1) Parr bomb combustion of the sludge, and 2) sodium peroxide/hydroxide fusion of the sludge.

\section{Method for Parr Bomb Combustion of Sludges}

The procedure used for the bomb combustion is outlined below.

1. Approximately $0.25 \mathrm{~g}$ of sludge was weighed into the combustion crucible.

2. $0.5 \mathrm{~mL}$ of mineral oil was added to the crucible with the sample.

3. $1 \mathrm{~mL}$ of $1 \mathrm{M}$ sodium hydroxide was placed on the bottom of the bomb.

4. The bomb was assembled, charged to $30 \mathrm{~atm}$. with UHP oxygen, and vented. This flush was repeated two more times to remove the nitrogen contribution from air. The 
bomb was charged to a final pressure of 30 atm with UHP oxygen, placed into a water bath and then the sample was ignited with an electronic spark.

5. The bomb was allowed to stand in the water bath for $4 \mathrm{~min}$. to condense combustion gases.

6. The bomb condensate was rinsed three times into a flask and diluted to $50 \mathrm{~mL}$ with water.

The resulting solution was analyzed by ion chromatography for anions.

\section{Method for Sodium Peroxide/Sodium Hydroxide Fusion of Sludges}

The procedure used for the fusion is outlined below.

1. Approximately $0.25 \mathrm{~g}$ of sludge was weighed into a nickel crucible.

2. $\quad 1.5 \mathrm{~g}$ of reagent grade sodium peroxide and $1 \mathrm{~g}$ of ultra pure sodium hydroxide was added to the crucible with the sample.

3. The crucible with sample and reagents was covered and placed in a muffle furnace set at $600^{\circ} \mathrm{C}$ for $15 \mathrm{~min}$.

4. The samples were removed from the furnace and allowed to cool for 3-4 min.

5. The cover, crucible, and fusion salts were rinsed with water into a flask and diluted to $50 \mathrm{~mL}$.

The final solution was analyzed by ion chromatography for anions.

It is important to note that a bomb combustion or fusion preparation of the MVST sludge samples yields total concentrations of the element measured. An example would be sulfate analysis. A water leach of the sludge will yield a sulfate concentration due to water soluble compounds containing sulfate while a bomb or fusion preparation of the sludge would yield a sulfate concentration due not only to the compounds containing sulfates (both water soluble and insoluble) but any compound containing sulfur. In other words the bomb and fusion preparations yield a total sulfur concentration rather than a total sulfate concentration. In theory, the same principle applies to any anion determined using the bomb or fusion preparation methods. 
The final anion measurement technique for all the sample preparation methods was ion chromatography. For simple water samples, without complex chemical matrix problems, the empirical analytical error for ion chromatography measurements ranges from $4-6 \%$ for concentrations above 10 times the detection limits to $20-40 \%$ near the detection limit. The measurement of anions present at concentration much lower $(<1 / 25)$ than other anionic species present may increase the overall error of the measurement.

\subsection{Radiochemical Analysis}

The only standard radiochemical methods useful for radioactive waste characterization are EPA Method 600/900.0, Gross Alpha and Beta Radioactivity in Drinking Water, and EPA Method 600/901.1: Gamma Emitting Radionuclides in Drinking Water. The EPA Method 600/905.0, Radioactive Strontium in Drinking Water, gave poor performance with the chemical matrix found in ORNL LLLW supernatant and sludge samples. The EPA method for gross alpha/beta measurements uses gas-flow proportional counting. In general, this counting technique requires drying a sample at elevated temperatures onto a metal (usually stainless steel) plate, which resulted in the loss of cesium chloride from the MVST samples and yielded poor gross beta results. To avoid this problem, all gross beta measurements reported are based on measurements by liquid scintillation counting. Other than the gamma spectroscopy measurements, all of the radionuclide measurements were done with in-house procedures. The method detection limits for radiochemical measurements are dependent on both sample matrix and count time and are not listed here. In general, the radiochemical measurements used count times to yield at least $1 \%$ (10,000 counts) counting statistics. The expected errors for the radiochemical data range from $\pm 5-10 \%$ for gross alpha/beta and gamma emitter measurements to $\pm 10-20 \%$ for radionuclides that require chemical separations before counting (i.e. ${ }^{99} \mathrm{Tc},{ }^{90} \mathrm{Sr},{ }^{129} \mathrm{I}$, and ${ }^{237} \mathrm{~Np}$ ).

The long-lived fission products are typically more difficult and expensive to measure than short lived fission products. Many of these long-lived radionuclides are either pure beta emitters or have weak, low energy, and/or low yield gamma-rays which are not very useful for accurate analytical measurements. In general, good radiochemical data requires that each of these isotopes be chemically separated from all other radioactivity prior to measurement. These chemical separations 
and measurements are currently being done routinely for ${ }^{99} \mathrm{Tc}$ and ${ }^{129} \mathrm{I}$ because both can exist as anionic species $\left(\mathrm{TcO}_{4}^{-}, \mathrm{I}^{-}\right.$, and $\left.\mathrm{IO}_{3}^{-}\right)$in the waste, and these anions would be highly mobile in the environment. The ${ }^{99} \mathrm{Tc}$ is currently being separated by extraction chromatography and measured by ICP-MS which is much more sensitive than counting techniques for radionuclides with a low specific activity. The ${ }^{129} \mathrm{I}$ is first extracted into carbon tetrachloride as iodine $\left(\mathrm{I}_{2}\right)$, then reduced to iodide $\left(\mathrm{I}^{-}\right)$, back-extracted into an aqueous matrix, and loaded onto an anion exchange resin. The ${ }^{129} \mathrm{I}$ is then determined by neutron activation analysis. Typically the level of ${ }^{99} \mathrm{Tc}$ and ${ }^{129} \mathrm{I}$ in the waste is lower than expected from the fission yields, and one possible explanation is that both isotopes form volatile species $\left(\mathrm{HTcO}_{4}, \mathrm{HI}\right.$, and $\left.\mathrm{I}_{2}\right)$ when exposed to either acid and/or heat.

The long-lived fission products are a very small fraction of the overall activity present in the waste and there has been little interest in the measurement of these radionuclides in the past. The determination of these isotopes are less routine and are frequently more expensive methods to. perform. The judgement of most waste characterization teams has been that the measurement of these radionuclides, with the exception of ${ }^{99} \mathrm{Tc}$, would be interesting but there is insufficient risk to justify the analytical cost.

\subsection{Criticality Controls}

The current ORNL waste acceptance criteria (WAC) for liquid-low level waste requires that the fissile isotopes of uranium and plutonium be isotopically diluted with ${ }^{238} \mathrm{U}$ and ${ }^{232} \mathrm{Th}$, respectively. These administrative controls require that the ratio of the ${ }^{238} U$ mass divided by the fissile equivalent mass (FEM) for uranium be greater than 100 . The ${ }^{235} \mathrm{U}$ FEM is a useful scale for criticality calculations that normalizes the fission probability for each fissile isotope to ${ }^{235} \mathrm{U}$. These FEM factors, designated as $f_{35}$ for ${ }^{235} \mathrm{U}$ mass factors, are discussed and listed in the Appendix A, Table 1 of ORNL Procedure NCS-1.0, Nuclear Criticality Safety Program.

The major fissile isotopes of concern in the ORNL waste tanks are ${ }^{233} \mathrm{U},{ }^{235} \mathrm{U}$, and ${ }^{239} \mathrm{Pu}$. The fissile isotope ${ }^{241} \mathrm{Pu}$ is also present in the waste but the mass is usually several orders of magnitude lower and below a level that would influence the isotopic dilution ratio for plutonium. Other fissile isotopes present in the ORNL waste include isotopes of neptunium, americium, and curium, but the 
actual mass present in the waste has been too low for major concern, and the low concentration would make it difficult and expensive to measure by mass spectrometry.

The data presented in this report for isotopic dilution ratios (also referred to as denature ratios) reflect both the past and current ORNL standard practices for disposal of fissile isotopes of uranium and plutonium. The administrative controls which were in effect when the waste was generated, required that the ${ }^{233} \mathrm{U}$ and ${ }^{235} \mathrm{U}$ be diluted with depleted uranium such that the following condition was true,

$$
\frac{\left({ }^{238} U\right)}{(1.35)\left({ }^{233} U\right)+\left({ }^{235} U\right)} \geq 100
$$

Because thorium chemistry is more similar to plutonium than uranium chemistry, the administrative procedures required that the ${ }^{239} \mathrm{Pu}$ be diluted with ${ }^{232} \mathrm{Th}$ as follows,

$$
\frac{(232 T h)}{\left({ }^{239} P u\right)} \geq 100
$$

All calculations dealing with isotopic dilution for criticality safety are based on isotope mass ratios and must not be confused with activity ratios. For any data discussed in this report that uses ${ }^{232} \mathrm{Th}$ relative to isotopic mass ratios, the total thorium concentration and the ${ }^{232} \mathrm{Th}$ concentration are the same value.

The new requirements for administrative criticality control, which should be in effect by the end of this year (1996), are more conservative and require that the following conditions be satisfied for uranium,

$$
\frac{(238 U)-200\left({ }^{233} U\right)}{\left({ }^{235} U\right)} \geq 110
$$




$$
\frac{\left({ }^{238} U\right)-100\left({ }^{235} U\right)}{\left({ }^{233} U\right)} \geq 200
$$

The new administrative controls also change requirements for plutonium by increasing the ratio of thorium to plutonium, as given in eq. 2 , from a dilution ratio of 100 to a ratio of 200 .

\subsection{Organic Analysis}

The organic sample preparation and analysis methods were based on SW-846 methods which had been adapted for radioactive samples. The performance of these methods had been demonstrated according to the Transuranic Waste Characterization Program (TWCP) Quality Assurance Program Plan (QAPP) ${ }^{9}$ requirements. The amounts of sample extracted and analyzed for this project were limited to ensure contamination control and good ALARA practices. There was some interference problems with the W-25 sludge sample which reduced the sensitivity of the semivolatile organic compound analysis (SVOA) by a factor of two. The sensitivities of the volatile organic compound analysis (VOA), the non-halogenated volatile organic compound analysis (NHVOA), and the polychlorinated biphenyls (PCB) analysis were not reduced for any measurements.

\subsubsection{Non-halogenated Volatile Organic Analysis (NHVOA)}

The NHVOA measurements were done by SW-846 Method 8015A, Nonhalogenated Volatile Organics by Gas Chromatography. One gram of sludge or one milliliter of supernatant was extracted by shaking with $1 \mathrm{~mL}$ of water. This extraction was reduced two-fold from the method used in the TWCP, but it retained the same method detection limit (MDL) because the relative proportions of sample and solvent were not changed. A volume of $0.001 \mathrm{~mL}$ of the extract was injected onto each of two gas chromatography columns, and the organic compounds were detected by flame ionization and quantified using the method of external standards. A surrogate standard was added to all samples and quality control samples. The latter included a laboratory blank, matrix spike (MS) and spike duplicate (MSD) samples, and a laboratory control sample (LCS). 


\subsubsection{Volatile Organic Analysis (VOA)}

The VOA measurements were done by SW-846 Method 8260A, Volatile Organic Compounds by Gas Chromatography/Mass Spectrometry (GC/MS): Capillary Column Technique. For sludge samples $1 \mathrm{~g}$ of solids was extracted by shaking with $1 \mathrm{~mL}$ of methanol. A $0.05 \mathrm{~mL}$ aliquot of the extract was added to $5 \mathrm{~mL}$ of water and was subjected to purge and trap gas chromatography-mass spectrometry (GC-MS). For the supernatant samples, the purge and trap GC-MS was done directly on $5 \mathrm{~mL}$ of each sample. Quantitation was by the method of internal standards. Surrogate standards were added to all samples and quality control samples. The latter included a laboratory blank, MS and MSD, and a LCS.

\subsubsection{Semivolatile Organic Analysis}

The SVOA measurements included SW-846 Method 3550A, Ultrasonic Extraction, for sample preparation, and SW-846 Method 8270B, Semivolatile Organic Compounds by Gas Chromatography / Mass Spectrometry (GC/MS): Capillary Column Technique, for sample analysis. For sludge samples, $10 \mathrm{~g}$ of solids were mixed with sodium sulfate until a free-flowing matrix was obtained, and the mixture was extracted with $100 \mathrm{~mL}$ of methylene chloride using an ultrasonic bath. For supernatant samples, $200 \mathrm{~mL}$ of liquid was extracted with $100 \mathrm{~mL}$ of methylene chloride according to SW-846 Method 3510, Separatory Funnel Liquid-Liquid Extraction. The methylene chloride was concentrated to $1 \mathrm{~mL}$, and the extract was analyzed by GC-MS using the method of internal standards. Surrogate standards were added to all samples and quality control samples. The latter included a laboratory blank, MS and MSD, and a LCS.

\subsubsection{Polychlorinated Biphenyls}

The PCB measurements included SW-846 Method 3550A, Ultrasonic Extraction and Method 3665, Sulfuric Acid/Permanganate Cleanup, for sample preparation, and Method 8081, Organochlorine Pesticides and PCBs as Aroclors by Gas Chromatography: Capillary Column Technique, for sample analysis. A fraction of the SVOA methylene chloride extract was used for the PCB sample preparation. The extract was concentrated and solvent-exchanged into hexane, washed with sulfuric acid until the acid washes were colorless and did not contain precipitates, washed with water to remove excess acid, combined with a hexane back-extract of the acid washes, and then were 
concentrated to $1 \mathrm{~mL}$. Analysis was conducted on a dual capillary column GC equipped with dual electron capture detectors using the method of external standards. A surrogate standard was added to all samples and quality control samples. The latter included a laboratory blank, MS and MSD, and a LCS. 


\subsection{Quality Assurance}

Both the inorganic and organic chemical characterization of the MVST samples followed the method requirements and Data Quality Objectives (DQO) of the TWCP QAPP. The RMAL implements the TWCP QAPP with a flow down RMAL Quality Assurance Project Plan (QAPjP) ${ }^{10}$ and implementation procedures. The list of metals determined was expanded from the TWCP requirements to meet ORNL needs. Although the organic target compounds were those listed in the TWCP QAPP, the full set of semivolatile and volatile organic compounds for the EPA Contract Laboratory Program Target Compound List (TCL) were reported as Tentatively Identified Compounds (TIC), if they were detected in the samples.

Quality assurance during the sampling activities was primarily addressed by the use of approved procedures for sampling both the liquid and sludge phase found in each waste tank. These procedures provide detailed instructions for the collection, labeling, and shipping of each sample. Chain-of-custody forms were used to track individual samples from their collection point to the analytical laboratory.

The RMAL also operates under a Radioactive Waste Characterization QA Plan ${ }^{11}$ which, in conjunction with the TWCP QAPjP, defines the basis for quality assurance and quality control used for the analysis of the waste tank samples. The QA plans discuss staff qualification requirements, laboratory participation in performance demonstration programs, quality control acceptance criteria for analytical methods, sample management, and most other laboratory operations. The set of QA plans implemented for RMAL waste characterization meet both the WIPP and the Nevada Test Site (NTS) QA requirements for inorganic, organic, and radiochemical measurements. 



\subsection{Summary of Inorganic and Radiochemical Analytical Results}

\subsection{Description of Data Tables}

A summary of the inorganic, physical, and radiochemical analytical results are presented in Table 3 and Table 4 for the MVST supernatant samples, and the data for MVST sludge samples are presented in Table 5 and Table 6. These tables are arranged in a similar format to facilitate comparing data from different tanks and to group information into useful units. The analytical data presented in these tables are the consolidation of data from a single project which had a fixed set of analytical requirements. Any parameter reported with a dash ("-") indicates that the data was not measured for that sample.

The first section, "Physical properties and miscellaneous data", includes unrelated information that does not fit well into other table groups. The first parameters entered in a column include the RMAL request and sample numbers, which are laboratory filing codes used to track sample information. The next set of data includes information on the moisture or water content and the solids content of the sample. The group is completed with data on the inorganic and organic carbon content. For MVST waste tank samples the inorganic carbon can be assumed to be all carbonate and bicarbonate. The Total Organic Carbon (TOC) provides an upper limit on the organic content in the tank waste but does not include volatile organic compounds. Most of the liquid waste in the active system has been through an evaporator which removes the highly volatile organic compounds from the waste.

The next two sections include groups of metals; the "RCRA metals" are separated out for quick reference. The regulatory limit for the concentrations are listed in parentheses next to each RCRA metal. For the liquid samples the RCRA regulatory limits are used directly, since the supernatant would be defined as the TCLP leachate in the determination of waste characteristics for hazardous waste. The RCRA metal sludge data represents total metal measurements, as defined by EPA. Exceeding the RCRA regulatory limits listed for the sludge samples only indicates that the waste has the potential to be classified as hazardous. The sludge waste should only be classified as RCRA waste if the final waste form fails the TCLP leaching test. 
The remaining metals are grouped under "Process metals", which includes the common Group IA \& IIA metals along with elements that could effect chemical processing, criticality concerns, and stabilization techniques such as grouting or vitrification. For the sludge data, all the metals are reported on a "as received" (wet weight) basis.

The section "Semi-quantitative metals by ICP-MS" includes additional metals identified in a full mass range scan by inductively-coupled plasma - mass spectrometry. This measurement helps ensure all major elements have been identified in the waste. Each element reported is not calibrated but is based upon a response factor from a curve generated from a few elements across the mass range. Therefore, these elemental concentrations are listed as estimates only.

The "Calculated Alkalinity" and the "Anions by ion chromatography" sections are separate for the supernatant samples, but are combined for the sludge samples. For supernatant samples the $\mathrm{pH}$ is measured directly, and the anions are determined on the liquid samples after dilution with water. The $\mathrm{pH}$ and anions reported for the sludge samples are based on a water wash of the sludge, as discussed in section 3.3. Along with the inorganic anions, several water soluble organic acids are reported, which includes compounds classified as complexing agents such as citrate and oxalate.

The "Beta/gamma emitters" section summarizes the radionuclides that emit gamma-rays and beta particles. This section includes the gross beta activity, radionuclides identified by gamma spectrometry, and several "pure" beta emitters of interest. Many of the "pure" beta emitters $\left({ }^{~}{ }^{\mathrm{H}},{ }^{14} \mathrm{C}\right.$, and ${ }^{90} \mathrm{Sr}$ ) require radiochemical separations prior to measurement by either liquid scintillation or gasflow proportional counting. The ${ }^{99} \mathrm{Tc}$ was measured by ICP-MS without any prior chemical separation and the ${ }^{151} \mathrm{Sm}$ were estimated by ICP-MS after a lanthanide group separation.

The "Alpha emitters" section summarize the actinide elements in the waste. These section includes the gross alpha activity, an estimate of the activity for each alpha emitter identified in a gross alpha spectrum, and plutonium isotopes determined by alpha spectrometry after a radiochemical separation. For supernatant samples, an estimate of the ${ }^{232} \mathrm{Th} /{ }^{239} \mathrm{Pu}$ mass ratio is included in this 
section to address criticality concerns if enough thorium is present to calculate the ratio. For the sludge samples, this mass ratio is included with the plutonium mass spectrometry data.

The remaining sections include "Uranium isotopes by TIMS", "Plutonium isotopes by TIMS", and "Uranium isotopes by ICP-MS". These sections summarize the uranium and plutonium data measured by thermal ionization mass spectrometry and for comparison to the uranium isotopes measured by ICP-MS. Also, included in these sections are the isotopic mass dilution or "denature" ratios for uranium and plutonium based on the requirements in place when the waste was generated (see section 3.5). The plutonium section for the sludge samples also includes the activity for each plutonium isotope, which was calculated from the mass spectrometry data. 
Table 3 Analytical Data for Liquids in Tanks W-24, W-25, and W-26

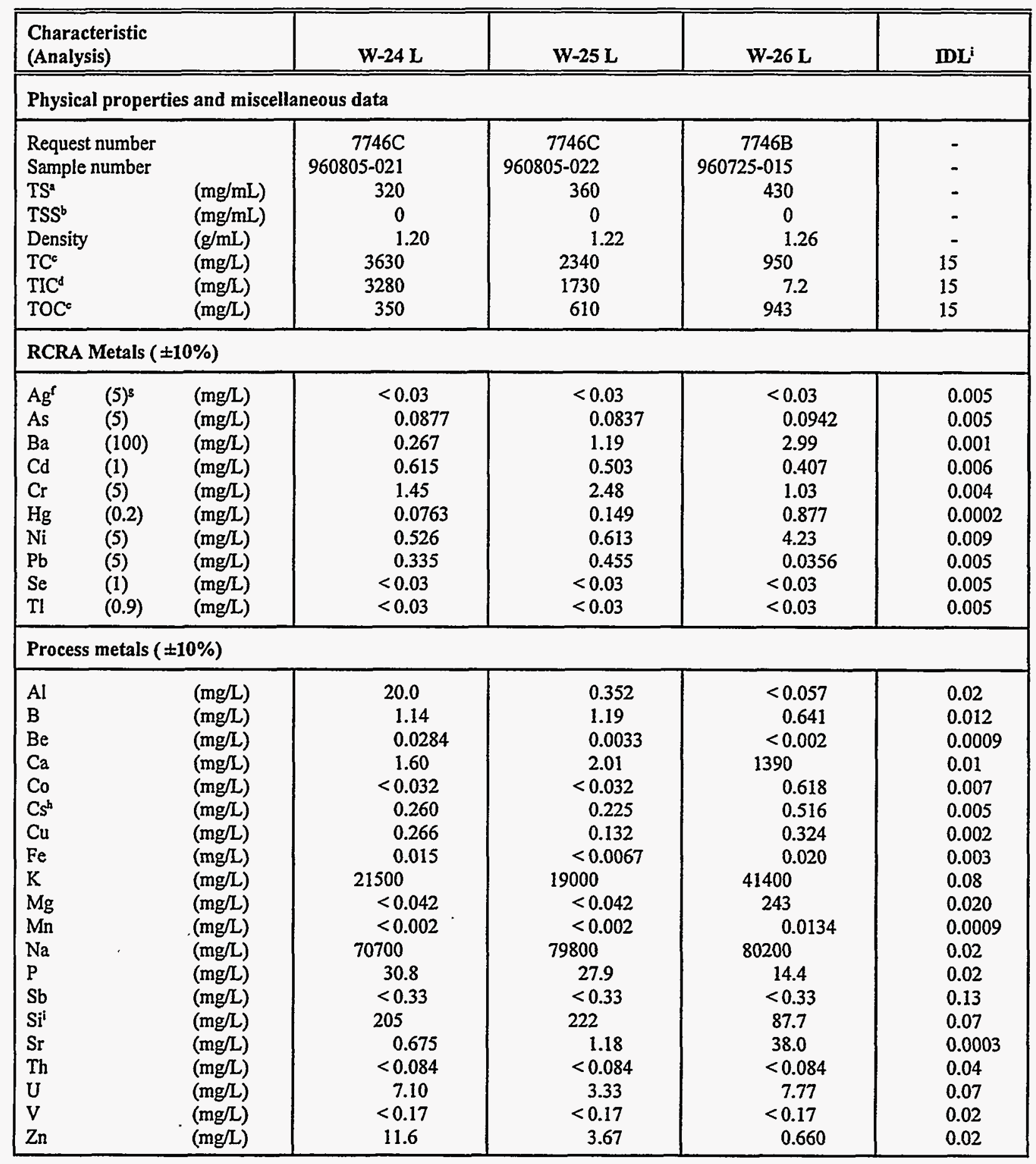




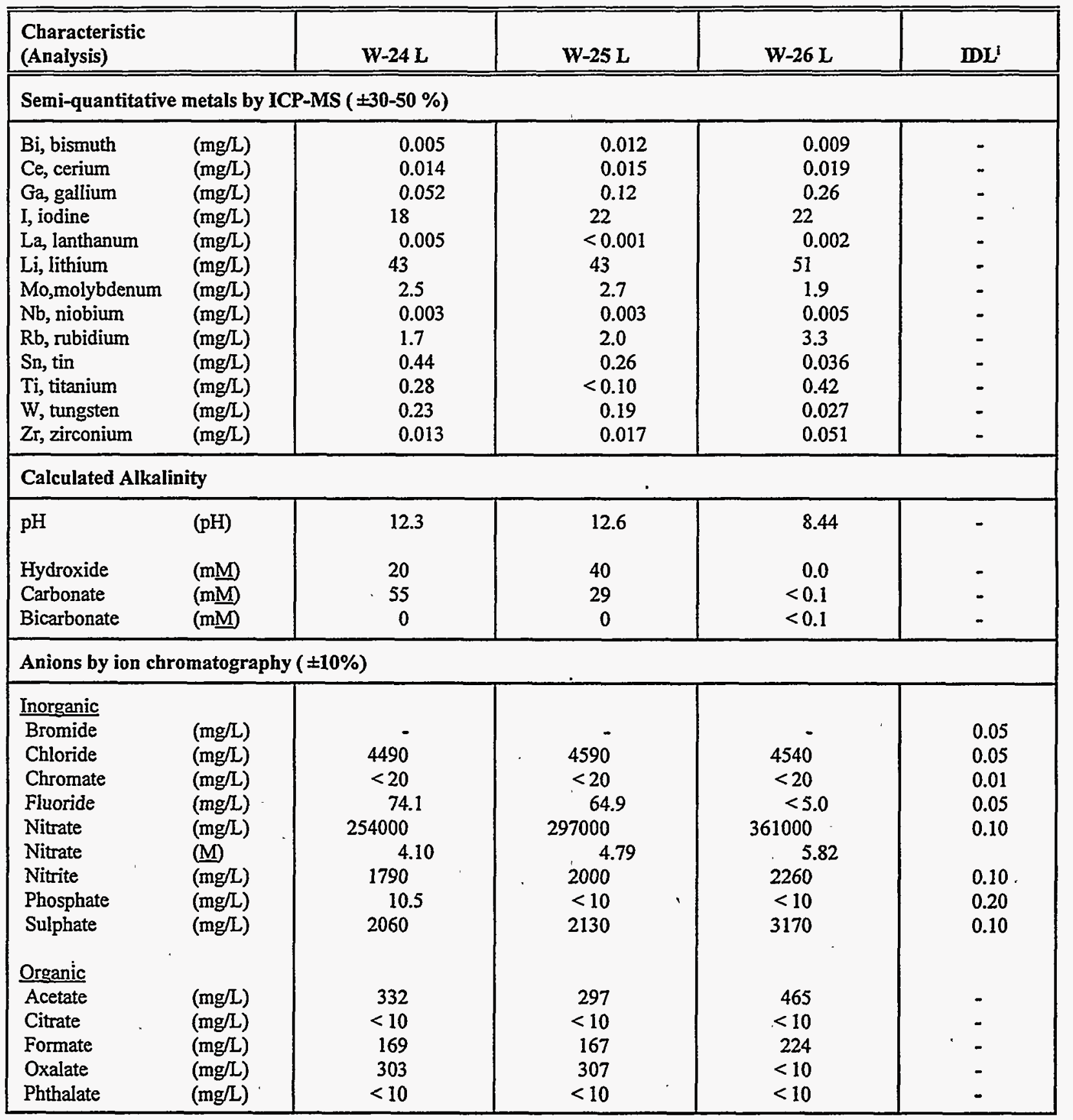




\begin{tabular}{|c|c|c|c|c|c|}
\hline $\begin{array}{l}\text { Characteristic } \\
\text { (Analysis) }\end{array}$ & & W-24 L & W-25 L & W-26 L & $\mathbf{W L} \mathbf{i}$ \\
\hline \multicolumn{6}{|c|}{ Beta/gamma emitters $( \pm 10 \%)$} \\
\hline $\begin{array}{l}\text { Gross beta } \\
{ }^{60} \mathrm{Co} \\
{ }^{90} \mathrm{Sr}{ }^{00} \mathrm{Y} \\
{ }^{90} \mathrm{Tc} \\
{ }^{129} \mathrm{I} \\
{ }^{134} \mathrm{Cs} \\
{ }^{137} \mathrm{Cs} \\
{ }^{152} \mathrm{Eu} \\
{ }^{154} \mathrm{Eu} \\
{ }^{155} \mathrm{Eu}\end{array}$ & $\begin{array}{l}(\mathrm{Bq} / \mathrm{mL}) \\
(\mathrm{Bq} / \mathrm{mL}) \\
(\mathrm{Bq} / \mathrm{mL}) \\
(\mathrm{Bq} / \mathrm{mL}) \\
(\mathrm{Bq} / \mathrm{mL}) \\
(\mathrm{Bq} / \mathrm{mL}) \\
(\mathrm{Bq} / \mathrm{mL}) \\
(\mathrm{Bq} / \mathrm{mL}) \\
(\mathrm{Bq} / \mathrm{mL}) \\
(\mathrm{Bq} / \mathrm{mL})\end{array}$ & $\begin{array}{r}1.2 e+06 \\
2.0 e+02 \\
5.8 e+03 \\
7.7 e+02 \\
2.5 e-01 \\
4.0 e+04 \\
1.1 e+06 \\
<6.0 e+02 \\
<4.0 e+02 \\
<2.0 e+03\end{array}$ & $\begin{array}{r}1.3 e+06 \\
1.8 e+02 \\
1.6 e+03 \\
7.4 e+02 \\
2.9 e-01 \\
4.5 e+04 \\
1.1 e+06 \\
<5.0 e+02 \\
<4.0 e+02 \\
<2.0 e+03\end{array}$ & $\begin{array}{r}1.7 e+06 \\
2.2 e+03 \\
2.5 e+04 \\
1.9 e+03 \\
7.8 e-02 \\
2.0 e+04 \\
1.4 e+06 \\
<9.0 e+02 \\
<5.0 e+02 \\
<2.0 e+03\end{array}$ & $\begin{array}{l}- \\
- \\
- \\
- \\
- \\
- \\
- \\
-\end{array}$ \\
\hline \multicolumn{6}{|c|}{ Alpha emitters $( \pm 10 \%)$} \\
\hline $\begin{array}{l}\text { Gross alpha } \\
{ }^{244} \mathrm{Cm} \\
{ }^{239} \mathrm{Pu} /{ }^{240} \mathrm{Pu} \\
{ }^{238} \mathrm{Pu} /{ }^{41} \mathrm{Am} \\
\text { Total } \mathrm{Pu} \text { alpha } \\
\frac{{ }^{238} \mathrm{Pu}}{{ }^{239} \mathrm{Pu} /{ }^{240} \mathrm{Pu}} \\
{ }^{42} \mathrm{Pu}\end{array}$ & $\begin{array}{l}(\mathrm{Bq} / \mathrm{mL}) \\
(\mathrm{Bq} / \mathrm{mL}) \\
(\mathrm{Bq} / \mathrm{mL}) \\
(\mathrm{Bq} / \mathrm{mL}) \\
\\
(\mathrm{Bq} / \mathrm{mL}) \\
(\mathrm{Bq} / \mathrm{mL}) \\
(\mathrm{Bq} / \mathrm{mL}) \\
(\mathrm{Bq} / \mathrm{mL})\end{array}$ & $\begin{array}{c}36 \\
- \\
- \\
- \\
\\
1.8 \\
1.1 \\
0.67 \\
0.01\end{array}$ & $\begin{array}{l}<1 \\
- \\
- \\
- \\
\\
0.96 \\
0.59 \\
0.35 \\
0.03\end{array}$ & $\begin{array}{r}77 \\
- \\
- \\
- \\
<1 \\
<1 \\
<1 \\
<1\end{array}$ & $\begin{array}{l}- \\
- \\
- \\
- \\
- \\
- \\
- \\
-\end{array}$ \\
\hline \multicolumn{6}{|c|}{ Uranium isotopics by TIMS ( $\pm 0.5 \%$ ) } \\
\hline $\begin{array}{l}{ }^{233} \mathrm{U} \\
{ }^{234} \mathrm{U} \\
{ }^{235} \mathrm{U} \\
{ }^{236} \mathrm{U} \\
{ }^{238} \mathrm{U} \\
{ }^{233} \mathrm{U} / \mathrm{MS} \\
{ }^{235} \mathrm{U} / \mathrm{MS} \\
{ }^{238} \mathrm{U} /{ }^{23} \mathrm{U} \text { FEM }\end{array}$ & $\begin{array}{l}\text { (atom \%) } \\
\text { (atom \%) } \\
\text { (atom \%) } \\
\text { (atom \%) } \\
\text { (atom \%) } \\
(\mathrm{ng} / \mathrm{mL} \text { ) } \\
(\mathrm{ng} / \mathrm{mL} \text { ) } \\
\text { - }\end{array}$ & $\begin{array}{r}0.11 \\
<0.01 \\
0.29 \\
0.01 \\
99.59 \\
\\
7.7 \\
20.3 \\
231\end{array}$ & $\begin{array}{r}0.11 \\
<0.01 \\
0.35 \\
0.01 \\
99.53 \\
\\
3.6 \\
11.5 \\
203\end{array}$ & $\begin{array}{r}0.14 \\
<0.01 \\
0.26 \\
<0.01 \\
99.60 \\
\\
10.6 \\
19.9 \\
225\end{array}$ & $\begin{array}{l}0.01 \\
0.01 \\
0.01 \\
0.01 \\
0.01 \\
- \\
- \\
-\end{array}$ \\
\hline $\begin{array}{l}\text { U activity } \\
{ }^{233} \mathrm{U} \\
{ }^{234} \mathrm{U} \\
{ }^{235} \mathrm{U} \\
{ }^{236} \mathrm{U} \\
{ }^{238} \mathrm{U}\end{array}$ & $\begin{array}{l}(\mathrm{Bq} / \mathrm{mL}) \\
(\mathrm{Bq} / \mathrm{mL}) \\
(\mathrm{Bq} / \mathrm{mL}) \\
(\mathrm{Bq} / \mathrm{mL}) \\
(\mathrm{Bq} / \mathrm{mL})\end{array}$ & $\begin{array}{r}2.7 \\
<0.1 \\
<0.1 \\
<0.1 \\
0.1\end{array}$ & $\begin{array}{r}\quad 1.3 \\
<0.1 \\
<0.1 \\
<0.1 \\
<0.1\end{array}$ & $\begin{array}{r}3.8 \\
<0.1 \\
<0.1 \\
<0.1 \\
0.1\end{array}$ & $\begin{array}{l}- \\
- \\
- \\
-\end{array}$ \\
\hline
\end{tabular}




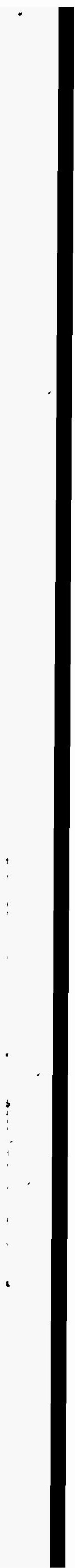


Table 4 Analytical Data for Liquids in Tanks W-27, W-28, and W-31

\begin{tabular}{|c|c|c|c|c|c|}
\hline \multicolumn{2}{|l|}{$\begin{array}{l}\text { Characteristic } \\
\text { (Analysis) }\end{array}$} & W-27 L & W-28 L & W-31 L & $\mathbf{m L}$ \\
\hline \multicolumn{6}{|c|}{ Physical properties and miscellaneous data } \\
\hline $\begin{array}{l}\text { Request number } \\
\text { Sample number } \\
\text { TS }^{a} \\
\text { TSS }^{b} \\
\text { Density } \\
\text { TC }^{c} \\
\text { TIC }^{d} \\
\text { TOC }^{c}\end{array}$ & $\begin{array}{l}(\mathrm{mg} / \mathrm{mL}) \\
(\mathrm{mg} / \mathrm{mL}) \\
(\mathrm{g} / \mathrm{mL}) \\
(\mathrm{mg} / \mathrm{L}) \\
(\mathrm{mg} / \mathrm{L}) \\
(\mathrm{mg} / \mathrm{L})\end{array}$ & $\begin{array}{c}7746 \mathrm{~B} \\
960725-016 \\
390 \\
0 \\
1.24 \\
401 \\
161 \\
240\end{array}$ & $\begin{array}{c}7746 \mathrm{~A} \\
960711-009 \\
580 \\
0 \\
1.34 \\
811 \\
36.3 \\
775\end{array}$ & $\begin{array}{c}7746 \mathrm{~A} \\
960711-028 \\
440 \\
0 \\
1.26 \\
1156 \\
407 \\
749\end{array}$ & $\begin{array}{r}- \\
- \\
- \\
- \\
- \\
15 \\
15 \\
15\end{array}$ \\
\hline \multicolumn{6}{|c|}{ RCRA Metals ( $\pm 10 \%)$} \\
\hline $\begin{array}{ll}\mathrm{Ag}^{\mathrm{r}} & (5)^{\mathrm{g}} \\
\mathrm{As} & (5) \\
\mathrm{Ba} & (100) \\
\mathrm{Cd} & (1) \\
\mathrm{Cr} & (5) \\
\mathrm{Hg} & (0.2) \\
\mathrm{Ni} & (5) \\
\mathrm{Pb} & (5) \\
\mathrm{Se} & (1) \\
\mathrm{Tl} & (0.9)\end{array}$ & $\begin{array}{l}(\mathrm{mg} / \mathrm{L}) \\
(\mathrm{mg} / \mathrm{L}) \\
(\mathrm{mg} / \mathrm{L}) \\
(\mathrm{mg} / \mathrm{L}) \\
(\mathrm{mg} / \mathrm{L}) \\
(\mathrm{mg} / \mathrm{L}) \\
(\mathrm{mg} / \mathrm{L}) \\
(\mathrm{mg} / \mathrm{L}) \\
(\mathrm{mg} / \mathrm{L}) \\
(\mathrm{mg} / \mathrm{L})\end{array}$ & $\begin{array}{c}<0.03 \\
<0.03 \\
5.21 \\
<0.03 \\
2.98 \\
0.288 \\
0.919 \\
0.0917 \\
<0.03 \\
<0.03\end{array}$ & $\begin{array}{l}<0.03 \\
<0.03 \\
6.13 \\
0.142 \\
0.499 \\
0.205 \\
1.37 \\
<0.03 \\
<0.03 \\
<0.03\end{array}$ & $\begin{array}{c}<0.03 \\
0.0316 \\
1.38 \\
0.431 \\
7.74 \\
2.28 \\
0.544 \\
0.154 \\
<0.03 \\
<0.03\end{array}$ & $\begin{array}{l}0.005 \\
0.005 \\
0.001 \\
0.006 \\
0.004 \\
0.0002 \\
0.009 \\
0.005 \\
0.005 \\
0.005\end{array}$ \\
\hline \multicolumn{6}{|c|}{ Process metals $( \pm 10 \%)$} \\
\hline $\begin{array}{l}\mathrm{Al} \\
\mathrm{B} \\
\mathrm{Be} \\
\mathrm{Ca} \\
\mathrm{Co} \\
\mathrm{Cs}^{\mathrm{h}} \\
\mathrm{Cu} \\
\mathrm{Fe} \\
\mathrm{K} \\
\mathrm{Mg} \\
\mathrm{Mn} \\
\mathrm{Na} \\
\mathrm{P} \\
\mathrm{Sb} \\
\mathrm{Si} \\
\mathrm{Sr} \\
\mathrm{Th} \\
\mathrm{U} \\
\mathrm{V} \\
\mathrm{Zn}\end{array}$ & $\begin{array}{l}(\mathrm{mg} / \mathrm{L}) \\
(\mathrm{mg} / \mathrm{L}) \\
(\mathrm{mg} / \mathrm{L}) \\
(\mathrm{mg} / \mathrm{L}) \\
(\mathrm{mg} / \mathrm{L}) \\
(\mathrm{mg} / \mathrm{L}) \\
(\mathrm{mg} / \mathrm{L}) \\
(\mathrm{mg} / \mathrm{L}) \\
(\mathrm{mg} / \mathrm{L}) \\
(\mathrm{mg} / \mathrm{L}) \\
(\mathrm{mg} / \mathrm{L}) \\
(\mathrm{mg} / \mathrm{L}) \\
(\mathrm{mg} / \mathrm{L}) \\
(\mathrm{mg} / \mathrm{L}) \\
(\mathrm{mg} / \mathrm{L}) \\
(\mathrm{mg} / \mathrm{L}) \\
(\mathrm{mg} / \mathrm{L}) \\
(\mathrm{mg} / \mathrm{L}) \\
(\mathrm{mg} / \mathrm{L}) \\
(\mathrm{mg} / \mathrm{L})\end{array}$ & $\begin{array}{c}<0.057 \\
0.428 \\
<0.002 \\
117 \\
<0.03 \\
0.104 \\
0.008 \\
<0.007 \\
9970 \\
0.331 \\
<0.002 \\
91100 \\
12.6 \\
<0.33 \\
41.0 \\
38.1 \\
<0.08 \\
0.708 \\
<0.17 \\
0.220\end{array}$ & $\begin{array}{c}2.99 \\
0.590 \\
<0.002 \\
9300 \\
0.0735 \\
0.161 \\
0.586 \\
0.175 \\
32200 \\
1760 \\
0.020 \\
117000 \\
7.23 \\
<0.33 \\
108 \\
81.3 \\
<0.08 \\
145 \\
<0.17 \\
0.421\end{array}$ & $\begin{array}{c}2.29 \\
0.593 \\
<0.002 \\
7.20 \\
<0.03 \\
0.202 \\
0.272 \\
0.0418 \\
16600 \\
1.33 \\
0.005 \\
103000 \\
28.9 \\
<0.33 \\
248 \\
1.35 \\
0.200 \\
60.8 \\
<0.17 \\
0.486\end{array}$ & $\begin{array}{l}0.02 \\
0.012 \\
0.0009 \\
0.01 \\
0.007 \\
0.005 \\
0.002 \\
0.003 \\
0.08 \\
0.020 \\
0.0009 \\
0.02 \\
0.02 \\
0.13 \\
0.07 \\
0.0003 \\
0.04 \\
0.07 \\
0.02 \\
0.02\end{array}$ \\
\hline
\end{tabular}




\begin{tabular}{|c|c|c|c|c|c|}
\hline $\begin{array}{l}\text { Characteristic } \\
\text { (Analysis) }\end{array}$ & & W-27 L & W-28 L & W-31 L & $\mathbf{I D L}^{\mathbf{i}}$ \\
\hline \multicolumn{6}{|c|}{ Semi-quantitative metals by ICP-MS ( $\pm 30-50 \%)$} \\
\hline $\begin{array}{l}\mathrm{Bi} \text {, bismuth } \\
\mathrm{Ce} \text {, cerium } \\
\mathrm{Ga} \text {, gallium } \\
\mathrm{I} \text {, iodine } \\
\mathrm{La} \text {, lanthanum } \\
\mathrm{Li} \text {, lithium } \\
\mathrm{Mo} \text {,molybdenum } \\
\mathrm{Nb} \text {, niobium } \\
\mathrm{Rb} \text {, rubidium } \\
\mathrm{Sn} \text {, tin } \\
\mathrm{Ti} \text {, titanium } \\
\mathrm{W} \text {, tungsten } \\
\mathrm{Zr} \text {, zirconium }\end{array}$ & $\begin{array}{l}(\mathrm{mg} / \mathrm{L}) \\
(\mathrm{mg} / \mathrm{L}) \\
(\mathrm{mg} / \mathrm{L}) \\
(\mathrm{mg} / \mathrm{L}) \\
(\mathrm{mg} / \mathrm{L}) \\
(\mathrm{mg} / \mathrm{L}) \\
(\mathrm{mg} / \mathrm{L}) \\
(\mathrm{mg} / \mathrm{L}) \\
(\mathrm{mg} / \mathrm{L}) \\
(\mathrm{mg} / \mathrm{L}) \\
(\mathrm{mg} / \mathrm{L}) \\
(\mathrm{mg} / \mathrm{L}) \\
(\mathrm{mg} / \mathrm{L})\end{array}$ & $\begin{array}{c}0.009 \\
<0.001 \\
0.48 \\
9.9 \\
0.007 \\
34 \\
0.95 \\
0.011 \\
1.4 \\
2.2 \\
0.40 \\
0.031 \\
0.045\end{array}$ & $\begin{array}{c}<0.001 \\
0.011 \\
0.53 \\
7.4 \\
0.002 \\
120 \\
0.79 \\
0.003 \\
3.1 \\
<0.001 \\
0.53 \\
0.011 \\
0.066\end{array}$ & $\begin{array}{l}0.017 \\
0.022 \\
0.13 \\
17 \\
<0.001 \\
27 \\
1.3 \\
0.003 \\
2.2 \\
3.1 \\
0.069 \\
0.052 \\
0.018\end{array}$ & $\begin{array}{l}- \\
- \\
- \\
- \\
- \\
- \\
- \\
- \\
- \\
-\end{array}$ \\
\hline \multicolumn{5}{|c|}{ Calculated Alkalinity } & . \\
\hline $\begin{array}{l}\mathrm{pH} \\
\text { Hydroxide } \\
\text { Carbonate } \\
\text { Bicarbonate }\end{array}$ & $\begin{array}{l}(\mathrm{pH}) \\
(\mathrm{mM}) \\
(\mathrm{mM}) \\
(\mathrm{mM})\end{array}$ & $\begin{array}{l}12.8 \\
63 \\
2.7 \\
0.0\end{array}$ & $\begin{array}{r}7.3 \\
\\
0.0 \\
<0.6 \\
<0.6\end{array}$ & $\begin{array}{l}10.0 \\
\\
0.1 \\
6.8 \\
0.0\end{array}$ & $\begin{array}{l}- \\
- \\
-\end{array}$ \\
\hline \multicolumn{6}{|c|}{ Anions by ion chromatography ( $\pm 10 \%)$} \\
\hline $\begin{array}{l}\text { Inorganic } \\
\text { Bromide } \\
\text { Chloride } \\
\text { Chromate } \\
\text { Fluoride } \\
\text { Nitrate } \\
\text { Nitrate } \\
\text { Nitrite } \\
\text { Phosphate } \\
\text { Sulphate } \\
\text { Organic } \\
\text { Acetate } \\
\text { Citrate } \\
\text { Formate } \\
\text { Oxalate } \\
\text { Phthalate }\end{array}$ & $\begin{array}{l}(\mathrm{mg} / \mathrm{L}) \\
(\mathrm{mg} / \mathrm{L}) \\
(\mathrm{mg} / \mathrm{L}) \\
(\mathrm{mg} / \mathrm{L}) \\
(\mathrm{mg} / \mathrm{L}) \\
(\mathrm{M}) \\
(\mathrm{mg} / \mathrm{L}) \\
(\mathrm{mg} / \mathrm{L}) \\
(\mathrm{mg} / \mathrm{L})\end{array}$ & $\begin{array}{c}- \\
3160 \\
<20 \\
<5 \\
340000 \\
5.48 \\
2070 \\
<10 \\
1510 \\
\cdot \\
145 \\
<10 \\
119 \\
<10 \\
<10\end{array}$ & $\begin{array}{c}5820 \\
<20 \\
<5 \\
506000 \\
8.16 \\
1430 \\
<10 \\
2070 \\
\\
\\
596 \\
<10 \\
208 \\
10.3 \\
<10\end{array}$ & $\begin{array}{c}- \\
4200 \\
56.2 \\
17.9 \\
391000 \\
6.31 \\
4430 \\
<10 \\
1850\end{array}$ & $\begin{array}{l}0.05 \\
0.05 \\
0.01 \\
0.05 \\
0.10 \\
- \\
0.10 \\
0.20 \\
0.10\end{array}$ \\
\hline
\end{tabular}




\begin{tabular}{|c|c|c|c|c|c|}
\hline $\begin{array}{l}\text { Characteristic } \\
\text { (Analysis) }\end{array}$ & & W-27 L & W-28 L & W-31 L & $\mathbf{I D L}^{\mathbf{i}}$ \\
\hline \multicolumn{6}{|c|}{ Beta/gamma emitters $( \pm 10 \%)$} \\
\hline $\begin{array}{l}\text { Gross beta } \\
{ }^{60} \mathrm{Co} \\
{ }^{90} \mathrm{Sr}{ }^{\circ 0} \mathrm{Y} \\
{ }^{99} \mathrm{Tc} \\
{ }^{129} \mathrm{I} \\
{ }^{134} \mathrm{Cs} \\
{ }^{137} \mathrm{Cs} \\
{ }^{152} \mathrm{Eu} \\
{ }^{154} \mathrm{Eu} \\
{ }^{155} \mathrm{Eu}\end{array}$ & $\begin{array}{l}(\mathrm{Bq} / \mathrm{mL}) \\
(\mathrm{Bq} / \mathrm{mL}) \\
(\mathrm{Bq} / \mathrm{mL}) \\
(\mathrm{Bq} / \mathrm{mL}) \\
(\mathrm{Bq} / \mathrm{mL}) \\
(\mathrm{Bq} / \mathrm{mL}) \\
(\mathrm{Bq} / \mathrm{mL}) \\
(\mathrm{Bq} / \mathrm{mL}) \\
(\mathrm{Bq} / \mathrm{mL}) \\
(\mathrm{Bq} / \mathrm{mL})\end{array}$ & $\begin{array}{r}4.4 e+05 \\
3.2 e+02 \\
9.5 e+04 \\
2.2 e+02 \\
1.4 e-02 \\
6.3 e+02 \\
2.8 e+05 \\
<8.0 e+01 \\
<9.0 e+01 \\
<5.0 e+02\end{array}$ & $\begin{array}{r}9.8 \mathrm{e}+05 \\
3.7 \mathrm{e}+03 \\
1.5 \mathrm{e}+05 \\
4.1 \mathrm{e}+02 \\
1.9 \mathrm{e}-02 \\
2.4 \mathrm{e}+03 \\
5.7 \mathrm{e}+05 \\
<2.0 \mathrm{e}+02 \\
<2.0 \mathrm{e}+02 \\
<6.0 \mathrm{e}+02\end{array}$ & $\begin{array}{r}5.4 e+05 \\
2.7 e+02 \\
1.5 e+04 \\
5.8 e+02 \\
6.5 e-02 \\
8.0 e+03 \\
4.3 e+05 \\
<2.0 e+02 \\
<2.0 e+02 \\
<6.0 e+02\end{array}$ & $\begin{array}{l}- \\
- \\
- \\
- \\
- \\
- \\
- \\
-\end{array}$ \\
\hline \multicolumn{6}{|c|}{ Alpha emitters $( \pm 10 \%)$} \\
\hline $\begin{array}{l}\frac{\text { Gross alpha }}{{ }^{244} \mathrm{Cm}} \\
{ }^{239} \mathrm{Pu} /{ }^{40} \mathrm{Pu} \\
{ }^{238} \mathrm{Pu} /{ }^{241} \mathrm{Am} \\
\text { Total Pu alpha } \\
{ }^{238} \mathrm{Pu} \\
{ }^{239} \mathrm{Pu} /{ }^{240} \mathrm{Pu} \\
{ }^{242} \mathrm{Pu}\end{array}$ & $\begin{array}{l}(\mathrm{Bq} / \mathrm{mL}) \\
(\mathrm{Bq} / \mathrm{mL}) \\
(\mathrm{Bq} / \mathrm{mL}) \\
(\mathrm{Bq} / \mathrm{mL}) \\
\\
(\mathrm{Bq} / \mathrm{mL}) \\
(\mathrm{Bq} / \mathrm{mL}) \\
(\mathrm{Bq} / \mathrm{mL}) \\
(\mathrm{Bq} / \mathrm{mL})\end{array}$ & $\begin{aligned} & 4.4 \\
& - \\
- & - \\
& \\
< & 1 \\
< & 1 \\
< & 1 \\
< & 1\end{aligned}$ & $\begin{array}{r}140 \\
- \\
- \\
- \\
<1 \\
<1 \\
<1 \\
<1\end{array}$ & $\begin{array}{c}32 \\
- \\
- \\
- \\
\\
6.2 \\
3.7 \\
2.5 \\
<0.1\end{array}$ & $\begin{array}{l}- \\
- \\
- \\
- \\
- \\
- \\
-\end{array}$ \\
\hline \multicolumn{6}{|c|}{ Uranium isotopics by TIMS ( $\pm 0.5 \%)$} \\
\hline $\begin{array}{l}{ }^{233} \mathrm{U} \\
{ }^{234} \mathrm{U} \\
{ }^{235} \mathrm{U} \\
{ }^{236} \mathrm{U} \\
{ }^{238} \mathrm{U} \\
{ }^{233} \mathrm{U} / \mathrm{MS} \\
{ }^{235} \mathrm{U} / \mathrm{MS} \\
{ }^{238} \mathrm{U} /{ }^{23} \mathrm{U} F \mathrm{FEM}\end{array}$ & $\begin{array}{l}\text { (atom \%) } \\
\text { (atom \%) } \\
\text { (atom \%) } \\
\text { (atom \%) } \\
\text { (atom \%) } \\
(\mathrm{ng} / \mathrm{mL}) \\
(\mathrm{ng} / \mathrm{mL}) \\
-\end{array}$ & $\begin{array}{r}0.10 \\
<0.01 \\
0.26 \\
<0.01 \\
99.63 \\
\\
0.7 \\
1.8 \\
256\end{array}$ & $\begin{array}{r}0.12 \\
<0.01 \\
0.24 \\
<0.01 \\
99.63 \\
\\
170 \\
344 \\
252\end{array}$ & $\begin{array}{c}0.11 \\
<0.01 \\
0.30 \\
0.01 \\
99.58 \\
\\
65.5 \\
180 \\
226\end{array}$ & $\begin{array}{l}0.01 \\
0.01 \\
0.01 \\
0.01 \\
0.01 \\
- \\
- \\
-\end{array}$ \\
\hline $\begin{array}{l}\text { U activity } \\
{ }^{233} \mathrm{U} \\
{ }^{234} \mathrm{U} \\
{ }^{235} \mathrm{U} \\
{ }^{236} \mathrm{U} \\
{ }^{238} \mathrm{U}\end{array}$ & $\begin{array}{l}(\mathrm{Bq} / \mathrm{mL}) \\
(\mathrm{Bq} / \mathrm{mL}) \\
(\mathrm{Bq} / \mathrm{mL}) \\
(\mathrm{Bq} / \mathrm{mL}) \\
(\mathrm{Bq} / \mathrm{mL})\end{array}$ & $\begin{array}{r}0.2 \\
<0.1 \\
<0.1 \\
<0.1 \\
<0.1\end{array}$ & $\begin{array}{r}60.8 \\
<0.1 \\
<0.1 \\
<0.1 \\
1.8\end{array}$ & $\begin{array}{r}23.4 \\
<0.1 \\
<0.1 \\
<0.1 \\
0.8\end{array}$ & $\begin{array}{l}- \\
- \\
- \\
-\end{array}$ \\
\hline $\begin{array}{l}\text { (a)Total solids, } \\
\text { inorganic carbo } \\
\text { ICP-MS, (i) nit }\end{array}$ & $\begin{array}{l}\text { SS is zero } \\
\text { Total orga } \\
\text { drofluoric }\end{array}$ & $\begin{array}{l}\text { uspended solid } \\
\text { n, (f) nitric-hy } \\
\text {, (j) Instrumen }\end{array}$ & $\begin{array}{l}\text { 1oved prior to } \\
\text { acid prep., (g) } \\
\text { limits. }\end{array}$ & $\begin{array}{l}\text { c) Total carbon } \\
\text { gulatory limits, }\end{array}$ & $\begin{array}{l}\text { al } \\
\text { sured by }\end{array}$ \\
\hline
\end{tabular}



Table 5 Analytical Data for Sludge in Tanks W-24, W-25, and W-26

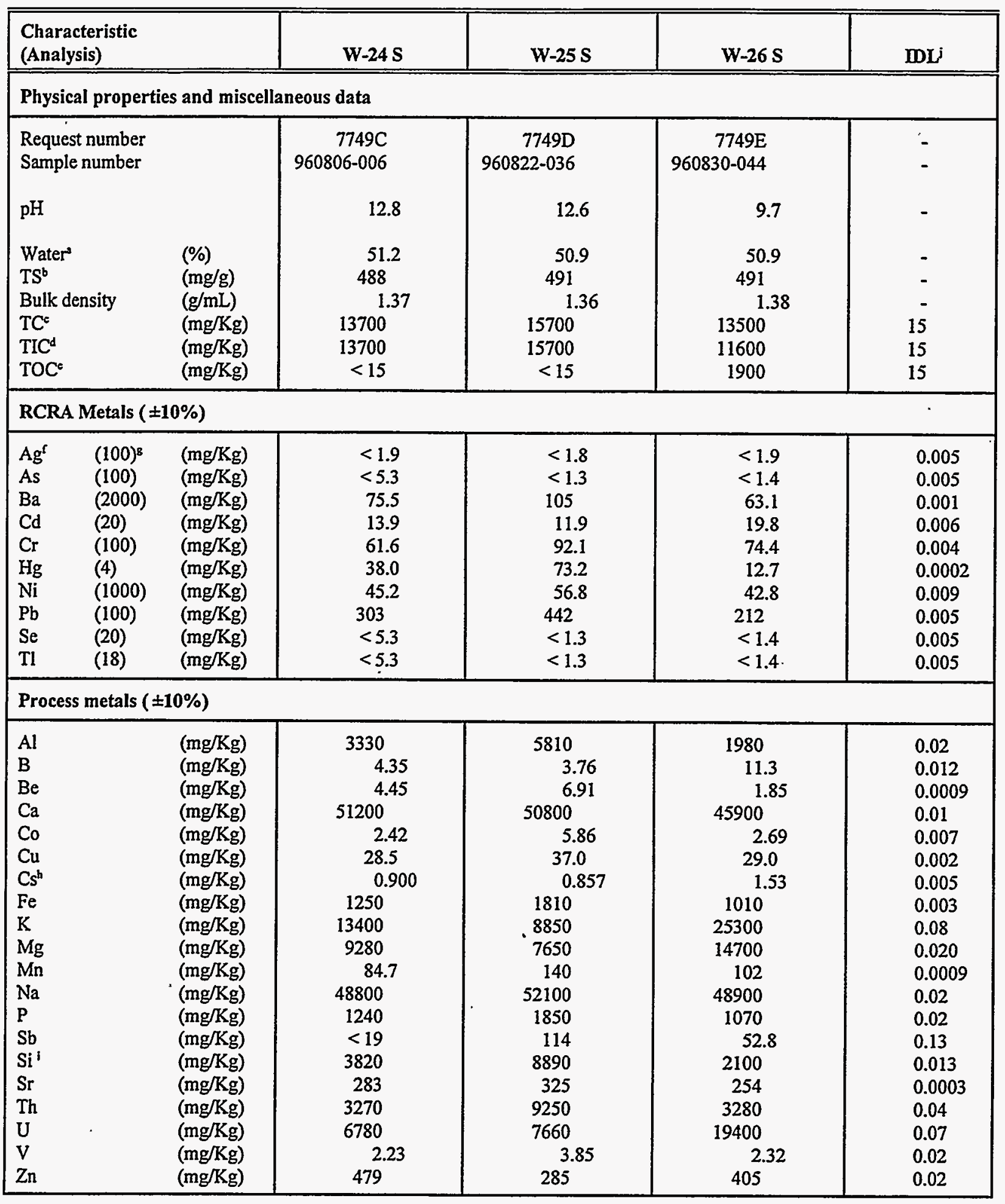




\begin{tabular}{|c|c|c|c|c|c|}
\hline $\begin{array}{l}\text { Characteristic } \\
\text { (Analysis) }\end{array}$ & & W-24 S & W-25 S & W-26 S & IDL \\
\hline \multicolumn{6}{|c|}{ Semi-quantitative metals by ICP-MS $( \pm 30-50 \%, *$ indicates data from water leach) } \\
\hline $\begin{array}{l}\mathrm{Au} \text {, gold } \\
\mathrm{Bi} \text {, bismuth } \\
\mathrm{Ce} \text {, cerium } \\
\mathrm{Er} \text {, erbium } \\
\text { Eu, europium } \\
\mathrm{Ga} \text {, gallium } \\
\mathrm{Gd} \text {, gadolinium } \\
\mathrm{Ho} \text {, holmium } \\
\mathrm{I} \text {, iodine } \\
\mathrm{La} \text {, lanthanum } \\
\mathrm{Li} \text {, lithium } \\
\mathrm{Mo} \text {,molybdenum } \\
\mathrm{Nb} \text {, niobium } \\
\mathrm{Rb} \text {, rubidium } \\
\mathrm{Sn} \text {, tin } \\
\mathrm{Ti} \text {, titanium } \\
\mathrm{W} \text {, tungsten } \\
\mathrm{Zr} \text {, zirconium }\end{array}$ & $\begin{array}{l}(\mathrm{mg} / \mathrm{Kg}) \\
(\mathrm{mg} / \mathrm{Kg}) \\
(\mathrm{mg} / \mathrm{Kg}) \\
(\mathrm{mg} / \mathrm{Kg}) \\
(\mathrm{mg} / \mathrm{Kg}) \\
(\mathrm{mg} / \mathrm{Kg}) \\
(\mathrm{mg} / \mathrm{Kg}) \\
(\mathrm{mg} / \mathrm{Kg}) \\
(\mathrm{mg} / \mathrm{Kg}) \\
(\mathrm{mg} / \mathrm{Kg}) \\
(\mathrm{mg} / \mathrm{Kg}) \\
(\mathrm{mg} / \mathrm{Kg}) \\
(\mathrm{mg} / \mathrm{Kg}) \\
(\mathrm{mg} / \mathrm{Kg}) \\
(\mathrm{mg} / \mathrm{Kg}) \\
(\mathrm{mg} / \mathrm{Kg}) \\
(\mathrm{mg} / \mathrm{Kg}) \\
(\mathrm{mg} / \mathrm{Kg})\end{array}$ & $\begin{array}{c}1.5 \\
170 \\
6.5 \\
0.25 \\
1.1 \\
5.3 \\
1.2 \\
1.0 \\
* 13 \\
9.1 \\
* 170 \\
* 2.1 \\
0.93 \\
* 1.4 \\
12 \\
21 \\
1.0 \\
8.4\end{array}$ & $\begin{array}{c}0.28 \\
250 \\
9.4 \\
0.02 \\
2.1 \\
8.1 \\
1.7 \\
2.0 \\
* 12 \\
18 \\
* 33 \\
* 2.0 \\
0.72 \\
* 1.0 \\
18 \\
47 \\
0.61 \\
16\end{array}$ & $\begin{array}{c}0.92 \\
78 \\
5.5 \\
0.24 \\
2.3 \\
4.0 \\
6.4 \\
1.0 \\
* 12 \\
4.8 \\
* 76 \\
* 2.2 \\
0.22 \\
* 2.5 \\
7.3 \\
3.2 \\
1.5 \\
5.4\end{array}$ & $\begin{array}{l}- \\
- \\
- \\
- \\
- \\
- \\
- \\
- \\
- \\
- \\
- \\
- \\
- \\
-\end{array}$ \\
\hline \multicolumn{6}{|c|}{ Anions by ion chromatography in water wash of sludge $( \pm 10 \%)$} \\
\hline $\begin{array}{l}\text { Inorganic } \\
\text { Bromide } \\
\text { Chloride } \\
\text { Chromate } \\
\text { Fluoride } \\
\text { Nitrate } \\
\text { Nitrite } \\
\text { Phosphate } \\
\text { Sulphate } \\
\text { Organic } \\
\text { Acetate } \\
\text { Citrate } \\
\text { Formate } \\
\text { Oxalate } \\
\text { Phthalate }\end{array}$ & $\begin{array}{l}(\mathrm{mg} / \mathrm{Kg}) \\
(\mathrm{mg} / \mathrm{Kg}) \\
(\mathrm{mg} / \mathrm{Kg}) \\
(\mathrm{mg} / \mathrm{Kg}) \\
(\mathrm{mg} / \mathrm{Kg}) \\
(\mathrm{mg} / \mathrm{Kg}) \\
(\mathrm{mg} / \mathrm{Kg}) \\
(\mathrm{mg} / \mathrm{Kg}) \\
\\
(\mathrm{mg} / \mathrm{Kg}) \\
(\mathrm{mg} / \mathrm{Kg}) \\
(\mathrm{mg} / \mathrm{Kg}) \\
(\mathrm{mg} / \mathrm{Kg}) \\
(\mathrm{mg} / \mathrm{Kg})\end{array}$ & $\begin{array}{r}<50 \\
2770 \\
<20 \\
103 \\
165000 \\
2250 \\
<20 \\
1370 \\
\\
\\
242 \\
<20 \\
175 \\
690 \\
<20\end{array}$ & $\begin{array}{c}<50 \\
2110 \\
95.5 \\
118 \\
162000 \\
4967 \\
<20 \\
1750 \\
\\
318 \\
<20 \\
247 \\
521 \\
<20\end{array}$ & $\begin{array}{c}<50 \\
3070 \\
<20 \\
<50 \\
214000 \\
1652 \\
<20 \\
2120 \\
\\
336 \\
<20 \\
243 \\
44.2 \\
<20\end{array}$ & $\begin{array}{l}0.05 \\
0.05 \\
0.01 \\
0.05 \\
0.10 \\
0.10 \\
0.20 \\
0.10 \\
- \\
- \\
- \\
- \\
-\end{array}$ \\
\hline \multicolumn{6}{|c|}{ Beta/gamma emitters $( \pm 10 \%)$} \\
\hline $\begin{array}{l}\frac{\text { Gross beta }}{{ }^{59} \mathrm{Ni}} \\
{ }^{63} \mathrm{Ni} \\
{ }^{60} \mathrm{Co} \\
{ }^{90} \mathrm{Sr}{ }^{90} \mathrm{Y} \\
{ }^{99} \mathrm{Tc} \\
{ }^{120} \mathrm{I} \\
{ }^{134} \mathrm{Cs} \\
{ }^{137} \mathrm{Cs} \\
{ }^{151} \mathrm{Sm} \\
{ }^{152} \mathrm{Eu} \\
{ }^{154} \mathrm{Eu} \\
{ }^{155} \mathrm{Eu} \\
{ }^{227} \mathrm{Ac} \\
{ }^{241} \mathrm{Pu}\end{array}$ & $\begin{array}{l}(\mathrm{Bq} / \mathrm{g}) \\
(\mathrm{Bq} / \mathrm{g}) \\
(\mathrm{Bq} / \mathrm{g}) \\
(\mathrm{Bq} / \mathrm{g}) \\
(\mathrm{Bq} / \mathrm{g}) \\
(\mathrm{Bq} / \mathrm{g}) \\
(\mathrm{Bq} / \mathrm{g}) \\
(\mathrm{Bq} / \mathrm{g}) \\
(\mathrm{Bq} / \mathrm{g}) \\
(\mathrm{Bq} / \mathrm{g}) \\
(\mathrm{Bq} / \mathrm{g}) \\
(\mathrm{Bq} / \mathrm{g}) \\
(\mathrm{Bq} / \mathrm{g}) \\
(\mathrm{Bq} / \mathrm{g}) \\
(\mathrm{Bq} / \mathrm{g})\end{array}$ & $\begin{array}{r}4.6 \mathrm{e}+06 \\
<2.5 \mathrm{e}+01 \\
3.3 \mathrm{e}+03 \\
2.8 \mathrm{e}+04 \\
1.4 \mathrm{e}+06 \\
4.5 \mathrm{e}+02 \\
- \\
1.3 \mathrm{e}+04 \\
5.3 \mathrm{e}+05 \\
<6.0 \mathrm{e}+02 \\
8.9 \mathrm{e}+04 \\
3.8 \mathrm{e}+04 \\
1.0 \mathrm{e}+04 \\
<4.7 \mathrm{e}+03 \\
1.4 \mathrm{e}+04\end{array}$ & $\begin{array}{r}8.3 e+06 \\
<2.5 \mathrm{e}+01 \\
3.4 \mathrm{e}+03 \\
2.5 \mathrm{e}+04 \\
3.2 \mathrm{e}+06 \\
1.0 \mathrm{e}+02 \\
- \\
6.0 \mathrm{e}+03 \\
4.7 \mathrm{e}+05 \\
<5.5 \mathrm{e}+02 \\
7.1 \mathrm{e}+04 \\
3.7 \mathrm{e}+04 \\
8.4 \mathrm{e}+03 \\
<5.3 \mathrm{e}+03 \\
2.6 \mathrm{e}+04\end{array}$ & $\begin{array}{r}3.5 e+06 \\
<3.0 e+01 \\
4.0 e+03 \\
5.8 e+04 \\
7.1 e+05 \\
1.2 e+03 \\
- \\
1.2 e+04 \\
8.9 e+05 \\
<5.8 e+02 \\
6.4 e+05 \\
2.9 e+05 \\
6.3 e+04 \\
<9.3 e+03 \\
1.5 e+04\end{array}$ & $\begin{array}{l}- \\
- \\
- \\
- \\
- \\
- \\
- \\
- \\
- \\
- \\
- \\
-\end{array}$ \\
\hline
\end{tabular}




\begin{tabular}{|c|c|c|c|c|c|}
\hline $\begin{array}{l}\text { Characteristic } \\
\text { (Analysis) }\end{array}$ & & W-24 S & W-25 S & $\mathrm{W}-26 \mathrm{~S}$ & $\mathbf{W L}^{\mathbf{j}}$ \\
\hline \multicolumn{6}{|c|}{ Alpha emitters $( \pm 10 \%)$} \\
\hline $\begin{array}{l}\text { Gross alpha } \\
{ }^{232} \mathrm{Th} \\
{ }^{233} \mathrm{U} \\
{ }^{234} \mathrm{U} \\
{ }^{235} \mathrm{U} \\
{ }^{238} \mathrm{U} \\
{ }^{237} \mathrm{~Np} \\
{ }^{241} \mathrm{Am} \\
{ }^{244} \mathrm{Cm} \\
{ }^{250} \mathrm{Cf} \\
{ }^{252} \mathrm{Cf} \\
\text { Total Pu alpha } \\
{ }^{238} \mathrm{Pu} \\
{ }^{239} \mathrm{Pu} / 240 \mathrm{Pu} \\
{ }^{242} \mathrm{Pu} \\
\text { TRU activity } \\
\text { PutAm (3700) }\end{array}$ & $\begin{array}{l}(\mathrm{Bq} / \mathrm{g}) \\
(\mathrm{Bq} / \mathrm{g}) \\
(\mathrm{Bq} / \mathrm{g}) \\
(\mathrm{Bq} / \mathrm{g}) \\
(\mathrm{Bq} / \mathrm{g}) \\
(\mathrm{Bq} / \mathrm{g}) \\
(\mathrm{Bq} / \mathrm{g}) \\
(\mathrm{Bq} / \mathrm{g}) \\
(\mathrm{Bq} / \mathrm{g}) \\
(\mathrm{Bq} / \mathrm{g}) \\
(\mathrm{Bq} / \mathrm{g}) \\
(\mathrm{Bq} / \mathrm{g}) \\
(\mathrm{Bq} / \mathrm{g}) \\
(\mathrm{Bq} / \mathrm{g}) \\
(\mathrm{Bq} / \mathrm{g})\end{array}$ & $\begin{array}{r}34000 \\
13 \\
1600 \\
77 \\
2.6 \\
1.0 \\
10 \\
3900 \\
22000 \\
<100 \\
<100 \\
6600 \\
4000 \\
2600 \\
-\end{array}$ & $\begin{array}{r}83000 \\
38 \\
2800 \\
100 \\
3.2 \\
1.1 \\
10 \\
9300 \\
58000 \\
<100 \\
<100 \\
13000 \\
7700 \\
4900 \\
- \\
22300\end{array}$ & $\begin{array}{c}52000 \\
13 \\
10000 \\
180 \\
4.0 \\
2.8 \\
2 \\
3900 \\
28000 \\
<100 \\
<100 \\
7600 \\
5300 \\
2300 \\
- \\
11500\end{array}$ & $\begin{array}{l}- \\
- \\
- \\
- \\
- \\
- \\
- \\
- \\
- \\
- \\
- \\
- \\
- \\
- \\
-\end{array}$ \\
\hline \multicolumn{6}{|c|}{ Uranium isotopics by TIMS ( $\pm 0.5 \%)$} \\
\hline $\begin{array}{l}{ }^{233} \mathrm{U} \\
{ }^{234} \mathrm{U} \\
{ }^{235} \mathrm{U} \\
{ }^{236} \mathrm{U} \\
{ }^{238} \mathrm{U} \\
{ }^{233} \mathrm{U} / \mathrm{MS} \\
{ }^{235} \mathrm{U} / \mathrm{MS} \\
{ }^{238} \mathrm{U} /{ }^{235} \mathrm{U} F \mathrm{FEM}\end{array}$ & $\begin{array}{l}\text { (atom \%) } \\
\text { (atom \%) } \\
\text { (atom \%) } \\
(\text { atom \%) } \\
\text { (atom \%) } \\
(\mathrm{mg} / \mathrm{Kg}) \\
(\mathrm{mg} / \mathrm{Kg}) \\
-\end{array}$ & $\begin{array}{r}0.054 \\
0.003 \\
0.496 \\
0.005 \\
99.442 \\
\\
3.58 \\
33.2 \\
177\end{array}$ & $\begin{array}{r}0.088 \\
0.006 \\
0.542 \\
0.006 \\
99.358 \\
\\
6.60 \\
45.2 \\
153\end{array}$ & $\begin{array}{r}0.132 \\
0.003 \\
0.268 \\
0.005 \\
99.592 \\
\\
25.1 \\
51.3 \\
227\end{array}$ & $\begin{array}{l}0.001 \\
0.001 \\
0.001 \\
0.001 \\
0.001 \\
- \\
- \\
-\end{array}$ \\
\hline \multicolumn{6}{|c|}{ Uranium isotopics by ICP-MS ( $\pm 2 \%)$} \\
\hline $\begin{array}{l}{ }^{233} \mathrm{U} \\
{ }^{234} \mathrm{U} \\
{ }^{23} \mathrm{U} \\
{ }^{236} \mathrm{U} \\
{ }^{238} \mathrm{U} \\
{ }^{233} \mathrm{U} / \mathrm{MS} \\
{ }^{235} \mathrm{U} / \mathrm{MS} \\
{ }^{238} \mathrm{U} /{ }^{233} \mathrm{U} \text { FEM }\end{array}$ & $\begin{array}{l}\text { (atom \%) } \\
\text { (atom \%) } \\
\text { (atom \%) } \\
\text { (atom \%) } \\
\text { (atom \%) } \\
(\mathrm{mg} / \mathrm{Kg}) \\
(\mathrm{mg} / \mathrm{Kg}) \\
-\end{array}$ & $\begin{array}{r}0.067 \\
0.005 \\
0.543 \\
0.006 \\
99.379 \\
\\
4.45 \\
36.4 \\
159\end{array}$ & $\begin{array}{r}0.103 \\
0.006 \\
0.597 \\
0.006 \\
99.289 \\
\\
7.72 \\
45.2 \\
137\end{array}$ & $\begin{array}{r}0.152 \\
0.004 \\
0.296 \\
0.006 \\
99.543 \\
\\
28.9 \\
56.7 \\
202\end{array}$ & $\begin{array}{l}0.001 \\
0.001 \\
0.001 \\
0.001 \\
0.001 \\
- \\
- \\
-\end{array}$ \\
\hline
\end{tabular}




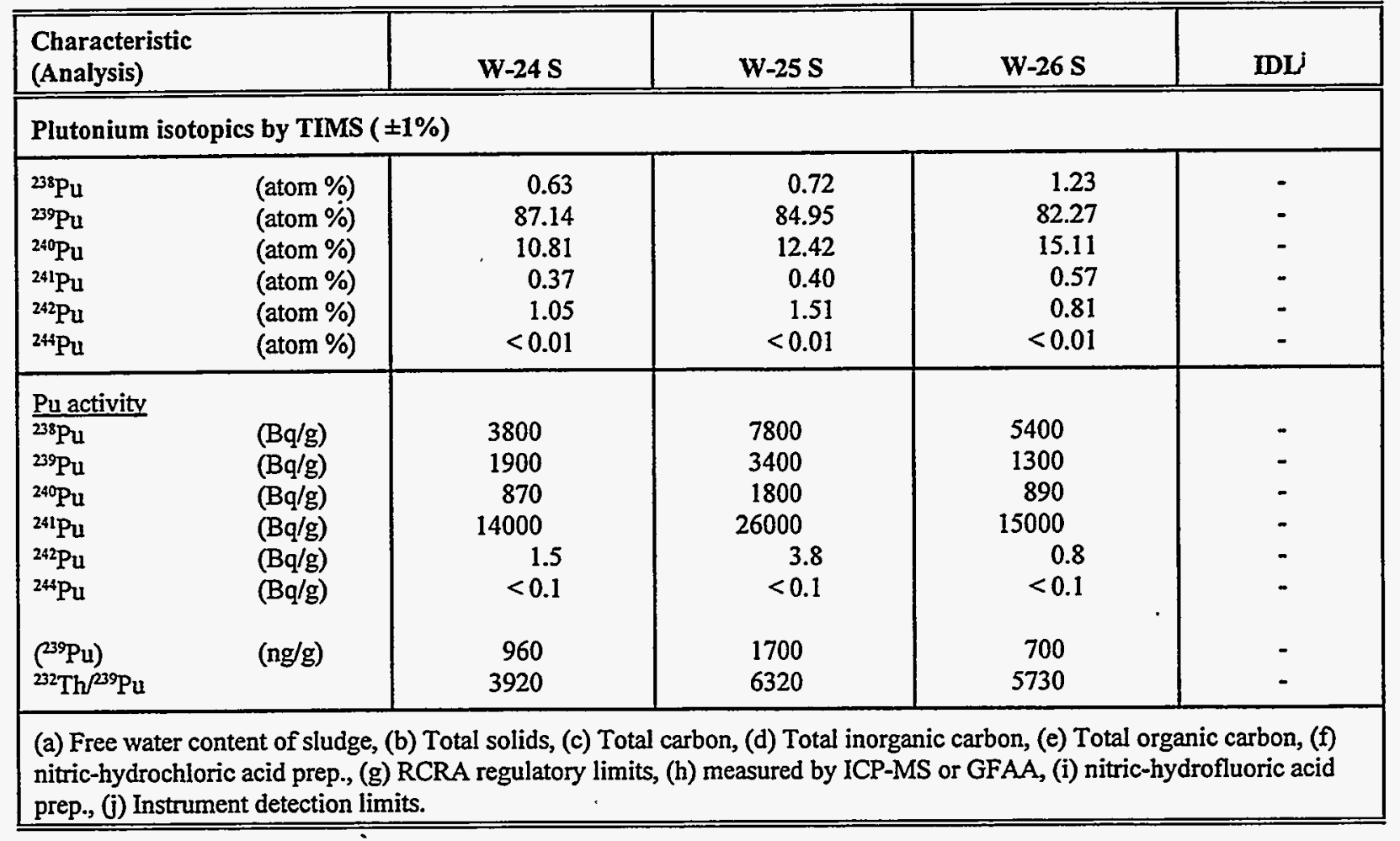


Table 6 Analytical Data for Sludge in Tanks W-27, W-28, and W-31

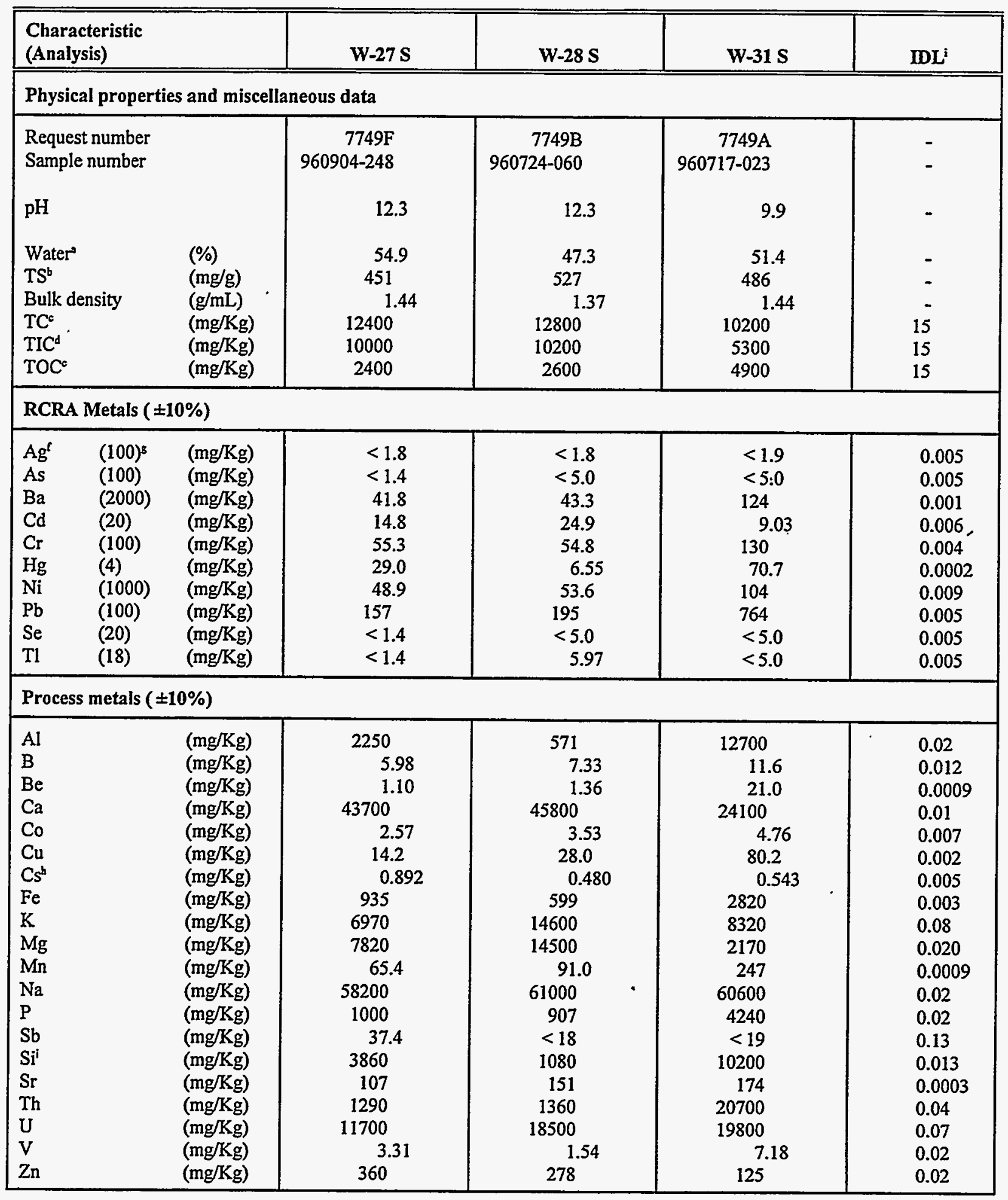




\begin{tabular}{|c|c|c|c|c|c|}
\hline $\begin{array}{l}\text { Characteristic } \\
\text { (Analysis) }\end{array}$ & - & $W-27 \mathrm{~S}$ & W-28 S & W-31 S & $\mathbf{m L}^{\mathbf{i}}$ \\
\hline \multicolumn{6}{|c|}{ Semi-quantitative metals by ICP-MS ( $\pm 30-50 \%, *$ indicates data from water leach) } \\
\hline $\begin{array}{l}\mathrm{Au} \text {, gold } \\
\mathrm{Bi} \text {, bismuth } \\
\mathrm{Ce} \text {, cerium } \\
\mathrm{Er} \text {, erbium } \\
\text { Eu, europium } \\
\mathrm{G} \text { a gailium } \\
\mathrm{Gd} \text {, gadolinium } \\
\mathrm{Ho} \text {, holmium } \\
\mathrm{I} \text {, iodine } \\
\mathrm{La} \text {, lanthanum } \\
\mathrm{Li} \text {, lithium } \\
\mathrm{Mo} \text {, molybdenum } \\
\mathrm{Nb} \text {, niobium } \\
\mathrm{Rb} \text {, rubidium } \\
\mathrm{Sn} \text {, tin } \\
\mathrm{Ti} \text {, titanium } \\
\mathrm{W} \text {, tungsten } \\
\mathrm{Zr} \text {, zirconium }\end{array}$ & $\begin{array}{l}(\mathrm{mg} / \mathrm{Kg}) \\
(\mathrm{mg} / \mathrm{Kg}) \\
(\mathrm{mg} / \mathrm{Kg}) \\
(\mathrm{mg} / \mathrm{Kg}) \\
(\mathrm{mg} / \mathrm{Kg}) \\
(\mathrm{mg} / \mathrm{Kg}) \\
(\mathrm{mg} / \mathrm{Kg}) \\
(\mathrm{mg} / \mathrm{Kg}) \\
(\mathrm{mg} / \mathrm{Kg}) \\
(\mathrm{mg} / \mathrm{Kg}) \\
(\mathrm{mg} / \mathrm{Kg}) \\
(\mathrm{mg} / \mathrm{Kg}) \\
(\mathrm{mg} / \mathrm{Kg}) \\
(\mathrm{mg} / \mathrm{Kg}) \\
(\mathrm{mg} / \mathrm{Kg}) \\
(\mathrm{mg} / \mathrm{Kg}) \\
(\mathrm{mg} / \mathrm{Kg}) \\
(\mathrm{mg} / \mathrm{Kg})\end{array}$ & $\begin{array}{c}0.62 \\
130 \\
7.2 \\
0.12 \\
0.80 \\
4.2 \\
1.9 \\
1.6 \\
* 6.8 \\
7.3 \\
* 53 \\
* 2.0 \\
0.56 \\
* 1.2 \\
4.0 \\
99 \\
1.3 \\
4.0\end{array}$ & $\begin{array}{c}1.9 \\
12 \\
7.9 \\
0.07 \\
1.5 \\
3.1 \\
6.0 \\
0.97 \\
* 9.1 \\
2.0 \\
* 170 \\
* 2.3 \\
0.30 \\
* 1.9 \\
5.9 \\
4.1 \\
1.4 \\
1.8\end{array}$ & $\begin{array}{c}2.6 \\
1200 \\
20 \\
0.85 \\
0.54 \\
12 \\
0.75 \\
0.22 \\
* 20 \\
54 \\
* 81 \\
* 1.4 \\
2.0 \\
* 1.3 \\
40 \\
34 \\
1.3 \\
51\end{array}$ & $\begin{array}{l}- \\
- \\
- \\
- \\
- \\
- \\
- \\
- \\
- \\
- \\
- \\
- \\
- \\
-\end{array}$ \\
\hline \multicolumn{6}{|c|}{ Anions by ion chromatography in water wash of sludge $( \pm 10 \%)$} \\
\hline $\begin{array}{l}\text { Inorganic } \\
\text { Bromide } \\
\text { Chloride } \\
\text { Chromate } \\
\text { Fluoride } \\
\text { Nitrate } \\
\text { Nitrite } \\
\text { Phosphate } \\
\text { Sulphate } \\
\text { Organic } \\
\text { Acetate } \\
\text { Citrate } \\
\text { Formate } \\
\text { Oxalate } \\
\text { Phthalate }\end{array}$ & $\begin{array}{l}(\mathrm{mg} / \mathrm{Kg}) \\
(\mathrm{mg} / \mathrm{Kg}) \\
(\mathrm{mg} / \mathrm{Kg}) \\
(\mathrm{mg} / \mathrm{Kg}) \\
(\mathrm{mg} / \mathrm{Kg}) \\
(\mathrm{mg} / \mathrm{Kg}) \\
(\mathrm{mg} / \mathrm{Kg}) \\
(\mathrm{mg} / \mathrm{Kg}) \\
\\
(\mathrm{mg} / \mathrm{Kg}) \\
(\mathrm{mg} / \mathrm{Kg}) \\
(\mathrm{mg} / \mathrm{Kg}) \\
(\mathrm{mg} / \mathrm{Kg}) \\
(\mathrm{mg} / \mathrm{Kg})\end{array}$ & $\begin{array}{c}<50 \\
2280 \\
<20 \\
<50 \\
210000 \\
2283 \\
<20 \\
549 \\
\\
196 \\
<20 \\
200 \\
16.0 \\
<20\end{array}$ & $\begin{array}{c}<50 \\
3460 \\
<20 \\
<50 \\
248000 \\
1120 \\
<20 \\
1773 \\
\\
325 \\
<20 \\
271 \\
19.1 \\
<20\end{array}$ & $\begin{array}{c}<50 \\
2570 \\
51.5 \\
125 \\
197000 \\
3470 \\
<50 \\
1090 \\
\\
237 \\
<50 \\
251 \\
89.8 \\
<50\end{array}$ & $\begin{array}{l}0.05 \\
0.05 \\
0.01 \\
0.05 \\
0.10 \\
0.10 \\
0.20 \\
0.10 \\
- \\
- \\
- \\
- \\
-\end{array}$ \\
\hline \multicolumn{6}{|c|}{ Beta/gamma emitters ( $\pm 10 \%)$} \\
\hline $\begin{array}{l}\frac{\text { Gross beta }}{{ }^{59} \mathrm{Ni}} \\
{ }^{63} \mathrm{Ni} \\
{ }^{60} \mathrm{Co} \\
{ }^{90} \mathrm{Sr} / 90 \mathrm{Y} \\
{ }^{99} \mathrm{Tc} \\
{ }^{129} \mathrm{I} \\
{ }^{134} \mathrm{Cs} \\
{ }^{137} \mathrm{Cs} \\
{ }^{151} \mathrm{Sm} \\
{ }^{152} \mathrm{Eu} \\
{ }^{154} \mathrm{Eu} \\
{ }^{155} \mathrm{Eu} \\
{ }^{227} \mathrm{Ac} \\
{ }^{241} \mathrm{Pu}\end{array}$ & $\begin{array}{l}(\mathrm{Bq} / \mathrm{g}) \\
(\mathrm{Bq} / \mathrm{g}) \\
(\mathrm{Bq} / \mathrm{g}) \\
(\mathrm{Bq} / \mathrm{g}) \\
(\mathrm{Bq} / \mathrm{g}) \\
(\mathrm{Bq} / \mathrm{g}) \\
(\mathrm{Bq} / \mathrm{g}) \\
(\mathrm{Bq} / \mathrm{g}) \\
(\mathrm{Bq} / \mathrm{g}) \\
(\mathrm{Bq} / \mathrm{g}) \\
(\mathrm{Bq} / \mathrm{g}) \\
(\mathrm{Bq} / \mathrm{g}) \\
(\mathrm{Bq} / \mathrm{g}) \\
(\mathrm{Bq} / \mathrm{g}) \\
(\mathrm{Bq} / \mathrm{g})\end{array}$ & $\begin{array}{r}1.6 \mathrm{e}+06 \\
<2.0 \mathrm{e}+01 \\
1.7 \mathrm{e}+03 \\
1.2 \mathrm{e}+04 \\
4.5 \mathrm{e}+05 \\
8.7 \mathrm{e}+01 \\
- \\
<8.1 \mathrm{e}+02 \\
3.9 \mathrm{e}+05 \\
<5.7 \mathrm{e}+02 \\
4.1 \mathrm{e}+04 \\
1.7 \mathrm{e}+04 \\
<2.7 \mathrm{e}+03 \\
<6.2 \mathrm{e}+03 \\
6.5 \mathrm{e}+03\end{array}$ & $\begin{array}{r}3.1 e+06 \\
<2.5 e+01 \\
3.3 e+03 \\
4.2 e+04 \\
7.0 e+05 \\
1.2 e+02 \\
4.1 e-02 \\
<1.2 e+03 \\
3.1 e+05 \\
<5.6 e+02 \\
8.0 e+05 \\
2.7 e+05 \\
7.0 e+04 \\
<6.7 e+03 \\
1.2 e+04\end{array}$ & $\begin{array}{r}2.4 \mathrm{e}+07 \\
<3.3 \mathrm{e}+01 \\
4.4 \mathrm{e}+03 \\
2.2 \mathrm{e}+04 \\
1.1 \mathrm{e}+07 \\
1.4 \mathrm{e}+02 \\
4.5 \mathrm{e}-02 \\
2.5 \mathrm{e}+03 \\
4.3 \mathrm{e}+05 \\
<6.0 \mathrm{e}+02 \\
3.0 \mathrm{e}+04 \\
2.0 \mathrm{e}+04 \\
<3.4 \mathrm{e}+03 \\
<5.8 \mathrm{e}+03 \\
2.4 \mathrm{e}+04\end{array}$ & $\begin{array}{l}- \\
- \\
- \\
- \\
- \\
- \\
- \\
- \\
- \\
- \\
- \\
- \\
-\end{array}$ \\
\hline
\end{tabular}




\begin{tabular}{|c|c|c|c|c|c|}
\hline $\begin{array}{l}\text { Characteristic } \\
\text { (Analysis) }\end{array}$ & & W-27 S & W-28 S & W-31 S & $\mathbf{D L}^{\mathbf{i}}$ \\
\hline \multicolumn{6}{|c|}{ Alpha emitters $( \pm 10 \%)$} \\
\hline $\begin{array}{l}\text { Gross alpha } \\
\frac{{ }^{232} \mathrm{Th}}{{ }^{233} \mathrm{U}} \\
{ }^{234} \mathrm{U} \\
{ }^{235} \mathrm{U} \\
{ }^{238} \mathrm{U} \\
{ }^{237} \mathrm{~Np} \\
{ }^{241} \mathrm{Am} \\
{ }^{244} \mathrm{Cm} \\
{ }^{250} \mathrm{Cf} \\
{ }^{252} \mathrm{Cf} \\
\text { Total Pu alpha } \\
{ }^{238} \mathrm{Pu} \\
{ }^{239} \mathrm{Pu} /{ }^{240} \mathrm{Pu} \\
{ }^{242} \mathrm{Pu} \\
\text { TRU activity } \\
\text { PutAm (3700) }\end{array}$ & 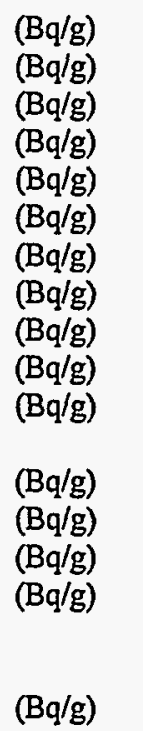 & $\begin{array}{c}26000 \\
5.2 \\
1000 \\
53 \\
2.5 \\
1.7 \\
12 \\
2800 \\
17000 \\
<100 \\
<100 \\
\\
3400 \\
2200 \\
1200 \\
- \\
\end{array}$ & $\begin{array}{c}44000 \\
5.5 \\
5200 \\
130 \\
3.8 \\
3.1 \\
16 \\
4600 \\
25000 \\
<100 \\
<100 \\
\\
4400 \\
2700 \\
1700 \\
- \\
9000\end{array}$ & $\begin{array}{c}160000 \\
84 \\
5200 \\
310 \\
10 \\
1.9 \\
21 \\
14000 \\
110000 \\
<100 \\
<100 \\
19000 \\
13000 \\
6200 \\
- \\
33000\end{array}$ & $\begin{array}{l}- \\
- \\
- \\
- \\
- \\
- \\
- \\
- \\
- \\
- \\
- \\
- \\
- \\
- \\
-\end{array}$ \\
\hline \multicolumn{6}{|c|}{ Uranium isotopics by TIMS ( $\pm 0.5 \%$ ) } \\
\hline $\begin{array}{l}{ }^{233} \mathrm{U} \\
{ }^{234} \mathrm{U} \\
{ }^{235} \mathrm{U} \\
{ }^{236} \mathrm{U} \\
{ }^{238} \mathrm{U} \\
{ }^{233} \mathrm{U} / \mathrm{MS} \\
{ }^{235} \mathrm{U} / \mathrm{MS} \\
{ }^{238} \mathrm{U} /{ }^{35} \mathrm{U} \text { FEM }\end{array}$ & $\begin{array}{l}\text { (atom \%) } \\
\text { (atom \%) } \\
\text { (atom \%) } \\
\text { (atom \%) } \\
\text { (atom \%) } \\
(\mathrm{mg} / \mathrm{Kg}) \\
(\mathrm{mg} / \mathrm{Kg}) \\
\text { - }\end{array}$ & $\begin{array}{c}0.022 \\
0.002 \\
0.309 \\
0.005 \\
99.662 \\
\\
2.52 \\
35.7 \\
298\end{array}$ & $\begin{array}{c}0.066 \\
0.003 \\
0.253 \\
0.007 \\
99.671 \\
\\
12.0 \\
46.2 \\
296\end{array}$ & $\begin{array}{r}0.056 \\
0.004 \\
0.621 \\
0.002 \\
99.316 \\
\\
10.9 \\
121 \\
145\end{array}$ & $\begin{array}{l}0.01 \\
0.01 \\
0.01 \\
0.01 \\
0.01 \\
- \\
- \\
-\end{array}$ \\
\hline \multicolumn{6}{|c|}{ Uranium isotopics by ICP-MS ( $\pm 2 \%$ ) } \\
\hline $\begin{array}{l}{ }^{233} \mathrm{U} \\
{ }^{234} \mathrm{U} \\
{ }^{235} \mathrm{U} \\
{ }^{236} \mathrm{U} \\
{ }^{238} \mathrm{U} \\
{ }^{233} \mathrm{U} / \mathrm{MS} \\
{ }^{235} \mathrm{U} / \mathrm{MS} \\
{ }^{238} \mathrm{U} /{ }^{235} \mathrm{U} \text { FEM }\end{array}$ & $\begin{array}{l}\text { (atom \%) } \\
\text { (atom \%) } \\
\text { (atom \%) } \\
\text { (atom \%) } \\
\text { (atom \%) } \\
(\mathrm{mg} / \mathrm{Kg}) \\
(\mathrm{mg} / \mathrm{Kg}) \\
\text { - }\end{array}$ & $\begin{array}{c}0.025 \\
0.002 \\
0.308 \\
0.006 \\
99.660 \\
\\
2.86 \\
35.6 \\
296\end{array}$ & $\begin{array}{c}0.081 \\
0.003 \\
0.296 \\
0.007 \\
99.613 \\
\\
14.7 \\
54.1 \\
249\end{array}$ & $\begin{array}{r}0.075 \\
0.007 \\
0.750 \\
0.004 \\
99.165 \\
\\
14.5 \\
150 \\
118\end{array}$ & $\begin{array}{l}0.01 \\
0.01 \\
0.01 \\
0.01 \\
0.01\end{array}$ \\
\hline
\end{tabular}




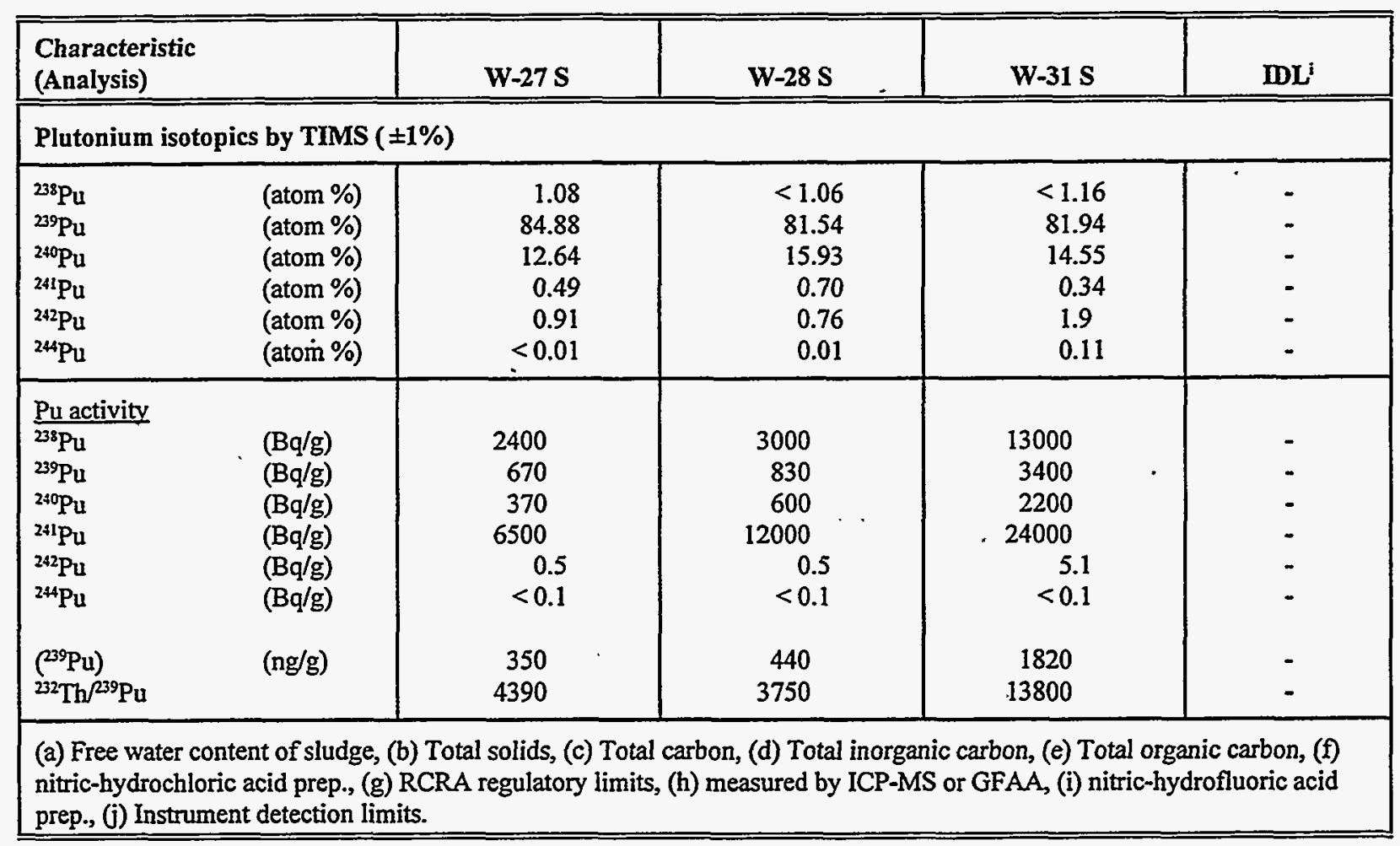




\subsection{Discussion of MVST Supernatant Characteristics}

The analytical data for the MVST supernatant samples are presented in Tables 3 and 4. This data is based on samples that were first clarified by centrifugation and then stabilized with nitric acid. Extra care was taken to remove suspended particles from the liquid samples because the particulate material was an artifact of the sampling technique and could bias the liquid phase data. If the $\mathrm{pH}$ is above 12 , most compounds of thorium, uranium and other actinide elements in the ORNL waste tanks form an insoluble precipitate. This chemical behavior is apparent with the supernatant data when the $\mathrm{pH}$ is compared to the uranium concentration and the alpha activity. For example, with tanks W-28 at $\mathrm{pH}=7.3$ and $\mathrm{W}-31$ at $\mathrm{pH}=10$, the uranium increases to $145 \mathrm{mg} / \mathrm{L}$ and $60.8 \mathrm{mg} / \mathrm{L}$, respectively. With tanks $\mathrm{W}-24, \mathrm{~W}-25$, and $\mathrm{W}-27$, where the $\mathrm{pH}>12$, the uranium concentration is less than $10 \mathrm{mg} / \mathrm{L}$. If higher levels of uranium are observed at $\mathrm{pH}>12$, it usually indicates that there are suspended particles of insoluble uranium present.

The Group IA elements, sodium and potassium, are very soluble in the supernatant at any $\mathrm{pH}$. In general, the concentration of Group IIA metals, calcium and strontium, increases in the supernatant as the $\mathrm{pH}$ decreases. These Group IIA metals remain somewhat soluble in the liquid phase, even at high $\mathrm{pH}$, unless the supernatant has absorbed some carbon dioxide from the air, which forms insoluble carbonate compounds with both calcium and strontium. The general distribution of radioactivity in the MVST tanks is a function of the $\mathrm{pH}$, where at higher $\mathrm{pH}$ the ${ }^{137} \mathrm{Cs}$ dominates the beta activity in the liquid phase and the ${ }^{90} \mathrm{Sr}{ }^{90} \mathrm{Y}$ is the predominate source of the beta activity in the sludge phase. At high $\mathrm{pH}$, the actinide elements are mostly insoluble which corresponds to most of the alpha activity being concentrated in the sludge phase.

As expected, the concentration of silicon compounds increases in the supernatant as the $\mathrm{pH}$ increases. Many of the other common metals found in the waste, such as iron and magnesium, are less soluble as the $\mathrm{pH}$ increases. In general, as the $\mathrm{pH}$ decreases, the total dissolved solids in the supernatant increases. Figure 1 and Fig. 2, illustrate the distribution of major cations and anions in the MVST liquid samples. Figure 2 is similar to Fig. 1, but with the sodium and nitrate removed to show more detail for species present at lower concentrations. 
Figure 1 Distribution of Major Cations and Anions in Liquid Phase MVST LIQUID

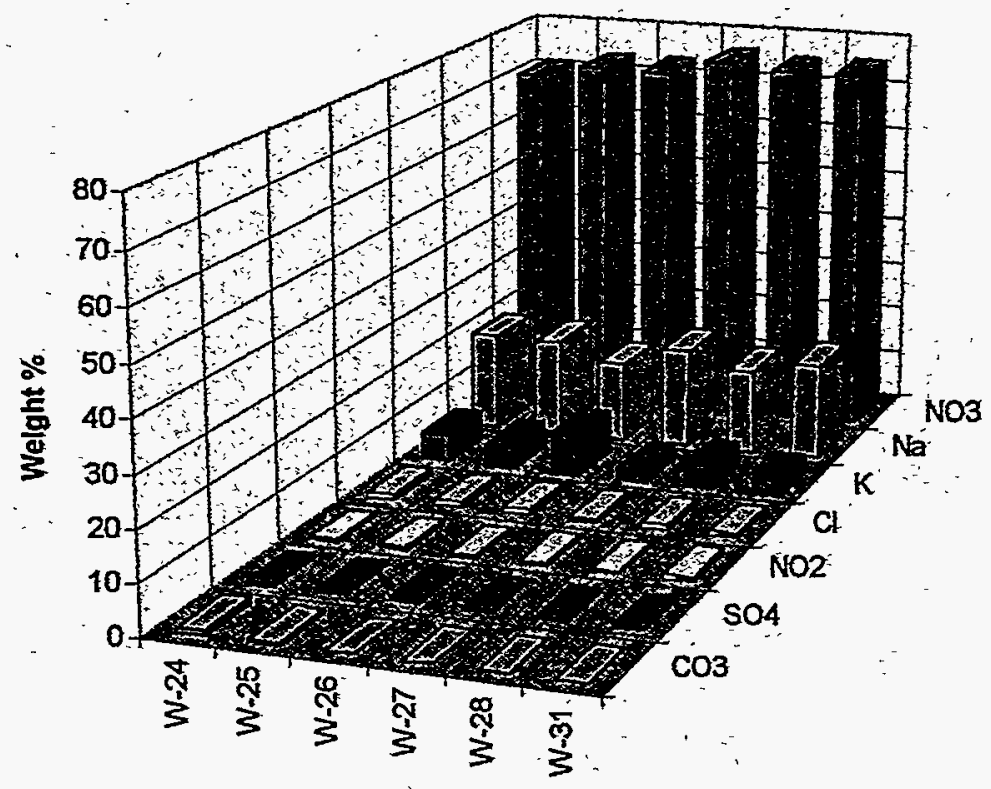

Figure 2 Distribution of Selected Ionic Species in Liquid Phase

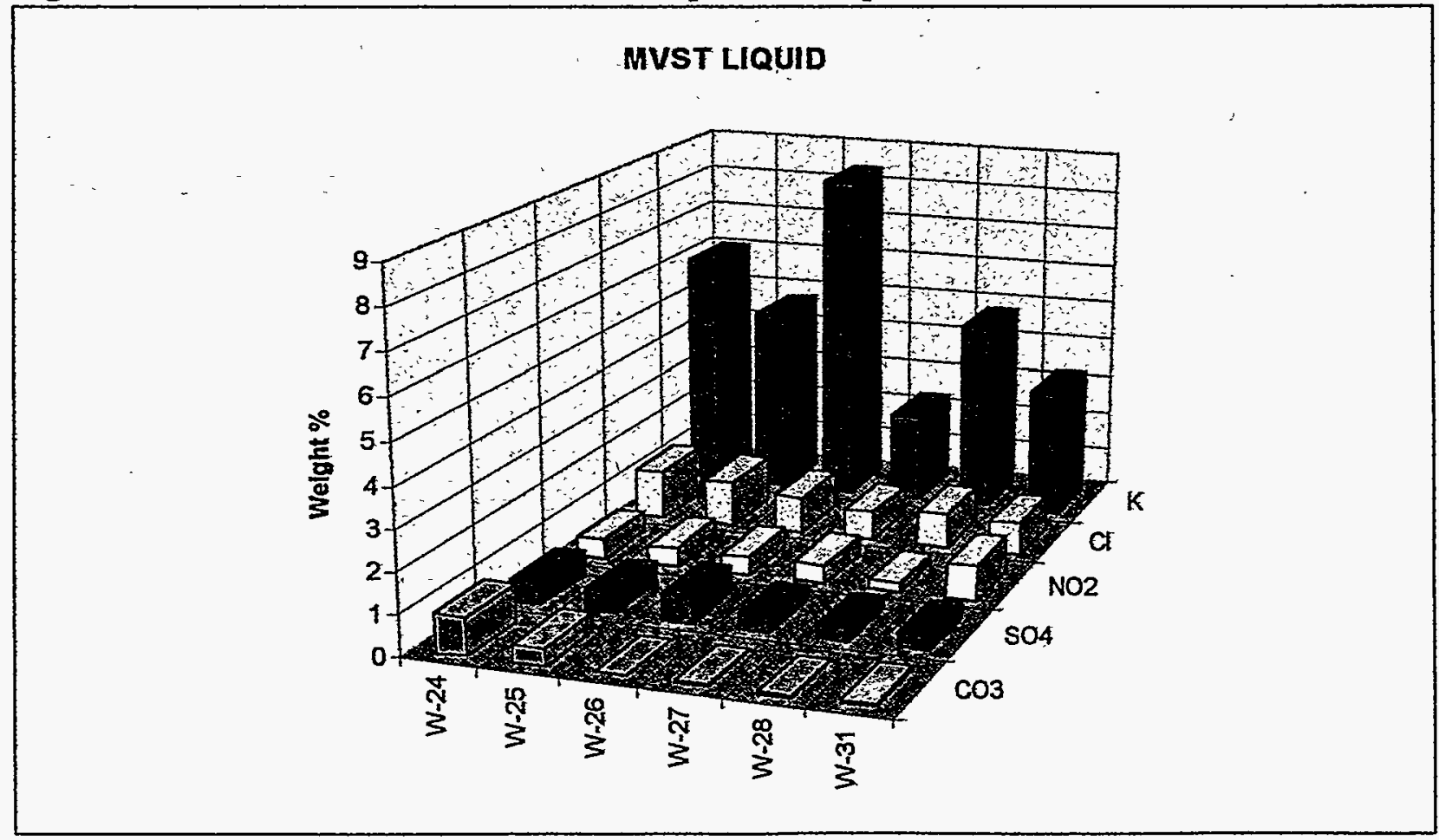


The sludge layers in the ORNL waste tanks are typically high in several RCRA metals, including chromium, mercury, and lead. At high $\mathrm{pH}$ these RCRA metals are below the hazard limits in the supernatant, but as the $\mathrm{pH}$ decreases the concentration of these RCRA metals can increase to the point where the regulatory limits are exceeded in the liquid phase.

A good check for data completeness is the mass and charge balance, which are summarized in Table 7 for the MVST supernatant samples. The mass balance check is based on the summation of cation and anion concentrations divided by the total solids concentration. The total solids concentration is measured directly by weighing a known volume of sample that has been dried to a constant weight. The mass balance data shows a high bias of $12-20 \%$ for the MVST supernatant samples. The charge balance checks are less accurate than the mass balance check because one must make an assumption about the chemical form and oxidation state for each species present in solution. The charge balance data is based on the summation of the molar cation charge divided by the summation of the molar anion charge. The charge balance data shows fair agreement but with a positive bias ranging from $22-34 \%$ for the MVST liquid samples. The charge balance data is acceptable considering the assumptions required for the calculation. The loss of nitrate as volatile oxides of nitrogen is one possible explanation for the high bias observed for both the total solids and the charge balance. For both the mass and charge balance checks on the supernatant samples, the calculations were dominated by the sodium, potassium, and nitrate concentration.

\section{Table 7 Summary of Quality Checks for MVST Supernatant Data}

\begin{tabular}{||c|c|c|c|c|c|c||}
\hline Tank & $\begin{array}{c}\text { Mass } \\
\text { Balance } \\
\left(\mathbf{T S}_{\text {calc }} / \mathbf{T S}_{\text {mess }}\right)\end{array}$ & $\begin{array}{c}\text { Charge } \\
\text { Balance } \\
\left(\mathbf{M}^{+} / \mathbf{A}^{-}\right)\end{array}$ & $\mathbf{p H}$ & $\begin{array}{c}{ }^{134} \mathbf{C s}^{+{ }^{137} \mathbf{C s}} \\
(\%)\end{array}$ & $\begin{array}{c}{ }^{90} \mathbf{S r} \mathbf{P}^{\circ} \mathbf{Y} \\
(\%)\end{array}$ & $\begin{array}{c}\text { Beta } \\
\text { Recovery } \\
(\%)\end{array}$ \\
\hline \hline W-24 & 1.12 & 1.22 & 12.3 & 99.06 & 0.87 & 111 \\
W-25 & 1.13 & 1.28 & 12.6 & 99.69 & 0.24 & 103 \\
W-26 & 1.15 & 1.31 & 8.4 & 96.83 & 2.93 & 101 \\
W-27 & 1.15 & 1.34 & 12.8 & 63.21 & 36.68 & 118 \\
W-28 & 1.17 & 1.29 & 7.3 & 68.70 & 30.87 & 99 \\
W-31 & 1.19 & 1.34 & 10.0 & 92.79 & 5.55 & 100 \\
\hline
\end{tabular}


The beta recovery listed in Table 7 is based on the summation of the activity for the known beta emitters divided by the gross beta activity. Considering the typical analytical errors associated which radiochemical measurements, the beta recoveries listed in Table 7 are excellent. The gross beta data reported is based on a total activity measurement by liquid scintillation counting which includes contributions from the conversion and Auger electrons. To determine the beta recovery, the total activity measurement minus the alpha activity is the gross beta value that is compared to the summation of the individual radionuclides identified. Also, one must take into account the large effect that analytical error for the radioactive strontium activity can have on the value of the beta recovery. Since the ${ }^{90} \mathrm{Sr}$ is in secular equilibrium with the ${ }^{90} \mathrm{Y}$, any error on the ${ }^{90} \mathrm{Sr}$ activity would be doubled when calculating the beta recovery.

Another point of interest in Table 7 is that the distribution of ${ }^{137} \mathrm{Cs}$ is independent of $\mathrm{pH}$, and that the ${ }^{90} \mathrm{Sr}$ activity is a function of both $\mathrm{pH}$ and carbonate concentration. At the most basic $\mathrm{pH}$ observed, almost all of the ${ }^{90} \mathrm{Sr}$ is precipitated into the sludge. As the basic environment becomes more acidic, the ${ }^{90} \mathrm{Sr}$ activity slowly increases in the supernatant. There was a significant jump in the relative ${ }^{90} \mathrm{Sr}$ activity observed in the supernatant when the $\mathrm{pH}$ dropped below $\mathrm{pH}=9$.

Tank W-27 appears to be an exception to this behavior, however, the elevated levels of radioactive strontium observed in the highly basic environment was due to the recent history with the tank chemistry. Tank W-27 had a pH in the same range as tank W-28 $(\mathrm{pH}=7.3)$ until one month prior to the sample being collected. Sodium hydroxide was added to tank W-27 until the $\mathrm{pH}$ was greater than $\mathrm{pH}=12$ and the supernatant was allowed to set undisturbed until the samples were taken for this project. The solubility of the strontium is dependent upon the concentration of carbonate, which was very low prior to the addition of the sodium hydroxide. There was insufficient time for the basic liquid to absorb carbon dioxide from the air and increase the carbonate concentration to a level that would precipitate the strontium (the calcium is also higher than expected at the high $\mathrm{pH}$ for the same reason).

Under conditions where the $\mathrm{pH}$ was high and the carbonate concentration was low, it is possible for the ${ }^{90} \mathrm{Sr}$ to remain soluble and the ${ }^{90} \mathrm{Y}$ to precipitate as the hydroxide and disrupt the secular 
equilibrium. It is important to understand any conditions that could disrupt this equilibrium because some radiochemical screening techniques and the interpretation of beta dose assume that the ${ }^{90} \mathrm{Y}$ activity is equal to the ${ }^{90} \mathrm{Sr}$ activity. The separation of the strontium from the yttrium is frequently observed with ${ }^{90} \mathrm{Sr}$ contaminated water moving through soil. The soluble ${ }^{90} \mathrm{Sr}$ moves with the water and the ${ }^{90} \mathrm{Y}$ is absorbed to the soil by an ion exchange process. Past practices used clay based materials as a mobilizing agent for pumping sludge. Therefore, the sludge may have an ion exchange affinity for yttrium or other radionuclides, which could interfere with the expected behavior for some radionuclides or other chemical species.

In general, the beta/gamma emitters found in the supernatant represent what would be expected for fission product waste that had been aged for several years. The relative distribution of the beta activity in the MVST supernatant is summarized in Table 8. The distribution of the activity in these MVST supernatant samples is typical of ORNL liquid waste. The ORNL liquid waste is normally stored at a caustic $\mathrm{pH}$ and the radioactive cesium dominates the activity. The $\mathrm{pH}$ in $\operatorname{tank} \mathrm{W}-28$ is lower than normal and there is a corresponding increase in the strontium and uranium in the liquid phase.

Table 8 Distribution of Beta Activity in Supernatant

\begin{tabular}{|c|c|c|c|c|c|c|}
\hline \multirow{2}{*}{ Tank } & \multirow{2}{*}{$\mathbf{p H}$} & \multicolumn{4}{|c|}{ Percent of Total Beta Activity } & \multirow{2}{*}{$\begin{array}{c}\text { Uranium } \\
\text { (mg/L) }\end{array}$} \\
\hline & & $\begin{array}{c}{ }^{90} \mathrm{Sr}^{\rho 0} \mathrm{Y} \\
(\%)\end{array}$ & $\begin{array}{l}{ }^{99} \mathrm{Tc} \\
(\%)\end{array}$ & $\begin{array}{l}{ }^{134} \mathrm{Cs} \\
(\%)\end{array}$ & $\begin{array}{l}{ }^{137} \mathrm{Cs} \\
(\%)\end{array}$ & \\
\hline W-24 & 12.3 & 0.87 & 0.06 & 3.04 & 96.02 & 7.1 \\
\hline $\mathrm{W}-25$ & 12.6 & 0.24 & 0.06 & 3.43 & 96.26 & 3.3 \\
\hline $\mathrm{W}-26$ & 8.4 & 2.93 & 0.11 & 1.19 & 95.64 & 7.8 \\
\hline W-27 & 12.8 & $36.68^{a}$ & 0.04 & 0.12 & 63.09 & 0.71 \\
\hline W-28 & 7.3 & 30.87 & 0.04 & 0.25 & 68.45 & 150 \\
\hline W-31 & 10.0 & 5.55 & 0.11 & 1.50 & 92.79 & 61 \\
\hline
\end{tabular}

${ }^{a}$ See previous discussion on tank W-27 concerning high strontium activity. 
The alpha activity in the supernatant is low, as would be expected with a caustic $\mathrm{pH}$. The small amount of alpha activity that is observed in the liquid phase can not be accounted for with the uranium present, however, the mass of the uranium present is much higher than the other actinide elements. Below $\mathrm{pH}=12$, the uranium forms a complex with the carbonate present and becomes more soluble as the $\mathrm{pH}$ decreases. As can be seen in Table 8, at the higher $\mathrm{pH}$ the uranium concentration is generally below $10 \mathrm{mg} / \mathrm{L}$, but in tank W-28 where the $\mathrm{pH}$ drops to 7.3 , the uranium concentration increases significantly.

The alpha content in the MVST supernatant is usually very low at higher $\mathrm{pH}$, but can increase if the $\mathrm{pH}$ is allowed to decrease too low. The alpha activity for the supernatant samples varies from $<1$ to $140 \mathrm{~Bq} / \mathrm{mL}$. Based on past experience, the alpha activity is likely due to suspended particles which are usually dominated by the ${ }^{244} \mathrm{Cm}$ alpha activity present. The uranium contribution to the total alpha activity is typically minor relative to the ${ }^{244} \mathrm{Cm}$ activity present in ORNL waste.

\subsection{Discussion of MVST Sludge Characteristics}

Determination of the mass and charge balance for the sludge samples are more difficult than for the supernatant samples. Not only are there assumptions required about the chemical form and the oxidation state of the species present in the sludge, but many of the compounds in the sludge are mixed oxides which are not directly measured. Also, the sludge is actually a slurry with a high water content. The interstitial liquid is in close contact with the sludge, and there are many ionic solubility equilibriums. The anion data for the sludge samples are based on the water soluble anions that would be available to a water wash. The water wash would not account for the insoluble hydroxides, carbonates, and mixed oxides present. The insoluble species do not contribute to the charge balance, and the cation charge is not used in the calculation, as indicated in Table 9. Most of the nitrate reported for the sludge is due to the interstitial liquid. Considering these limitations, the compounds listed in Table 9 were used to estimate the mass and charge balance. 
Table 9 Assumption Used for Major Compounds in MVST Sludge

\begin{tabular}{|c|c|c|c|}
\hline Cation & Chemical Form & $\begin{array}{c}\text { Cation } \\
\text { Charge Used }\end{array}$ & $\begin{array}{c}\text { Gravimetric } \\
\text { Factors }\end{array}$ \\
\hline \hline $\mathrm{Al}^{3+}$ & $\mathrm{Al}_{2} \mathrm{O}_{3}$ & 0 & 1.890 \\
$\mathrm{Ca}^{2+}$ & $\mathrm{CaCO}_{3}$ & 0 & 2.497 \\
$\mathrm{Fe}^{3+}$ & $\mathrm{Fe}_{2} \mathrm{O}_{3}$ & 0 & 1.430 \\
$\mathrm{~K}^{+}$ & $\mathrm{K}^{+} \mathrm{NO}_{3}^{-}$ & +1 & 2.586 \\
$\mathrm{Mg}^{2+}$ & $\mathrm{Mg}(\mathrm{OH})_{2}$ & 0 & 2.399 \\
$\mathrm{Mn}^{2+}$ & $\mathrm{Mn}(\mathrm{OH})_{2}$ & 0 & 1.619 \\
$\mathrm{Na}^{+}$ & $\mathrm{Na} \mathrm{NO}_{3}^{-}$ & +1 & 3.697 \\
$\mathrm{Th}^{4+}$ & $\mathrm{Th}(\mathrm{OH})_{4}$ & 0 & 1.293 \\
$\mathrm{UO}_{2}^{2+}$ & $\mathrm{UO}_{2}\left((\mathrm{OH})_{2}-\mathrm{H}_{2} \mathrm{O}\right.$ & 0 & 1.353 \\
\hline
\end{tabular}

Table 10 summarizes the mass and charge balance for the MVST tank sludge samples. Considering the limitations of these calculations, the mass balance is within the analytical error $( \pm 20 \%)$ for these sludge samples. The charge balance is more influenced by the chemical form assumptions, and the results have a larger corresponding error range.

Table 10 Summary of Quality Checks for MVST Sludge Data

\begin{tabular}{|c|c|c|c|c|c|c||}
\hline Tank & $\begin{array}{c}\text { Mass } \\
\text { Balance } \\
\left(\mathbf{T S}_{\text {calc }} / \mathbf{T S}_{\text {mess }}\right)\end{array}$ & $\begin{array}{c}\text { Charge } \\
\text { Balance } \\
\left(\mathbf{M}^{+} / \mathbf{A}^{-}\right)\end{array}$ & $\mathbf{p H}$ & $\begin{array}{c}{ }^{134} \mathrm{Cs}^{1{ }^{137}} \mathbf{C s} \\
(\%)\end{array}$ & $\begin{array}{c}{ }^{90} \mathrm{Sr}^{90} \mathbf{Y} \\
(\%)\end{array}$ & $\begin{array}{c}\text { Beta } \\
\text { Recovery } \\
(\%)\end{array}$ \\
\hline \hline W-24 & 0.831 & 0.752 & 12.8 & 17.4 & 77.3 & 79.4 \\
W-25 & 0.879 & 0.745 & 12.6 & 7.8 & 89.6 & 87.0 \\
W-26 & 0.908 & 0.693 & 9.7 & 29.3 & 39.6 & 104.0 \\
W-27 & 0.889 & 0.705 & 12.3 & 31.7 & 62.7 & 91.2 \\
W-28 & 0.857 & 0.673 & 12.3 & 12.2 & 47.3 & 96.8 \\
W-31 & 0.928 & 0.807 & 9.9 & 2.2 & 97.2 & 94.9 \\
\hline
\end{tabular}


The beta recovery results are listed in Table 10 , and most of the discussion for the supernatant samples also applies to the sludge samples. As discussed before, the variability for the beta recovery is probably due to the analytical error on the ${ }^{90} \mathrm{Sr}$ measurement. Any measurement error for the ${ }^{90} \mathrm{Sr}$ activity would be doubled when considering the beta recovery calculation.

The distribution, by weight percent, of the major compounds from Table 9 are illustrated in Fig. 3 for each MVST sludge sample. The distribution of the total uranium and thorium concentration for each MVST sludge sample are shown in Fig. 4. 
Figure 3 Distribution of Major Compounds in MVST Sludge

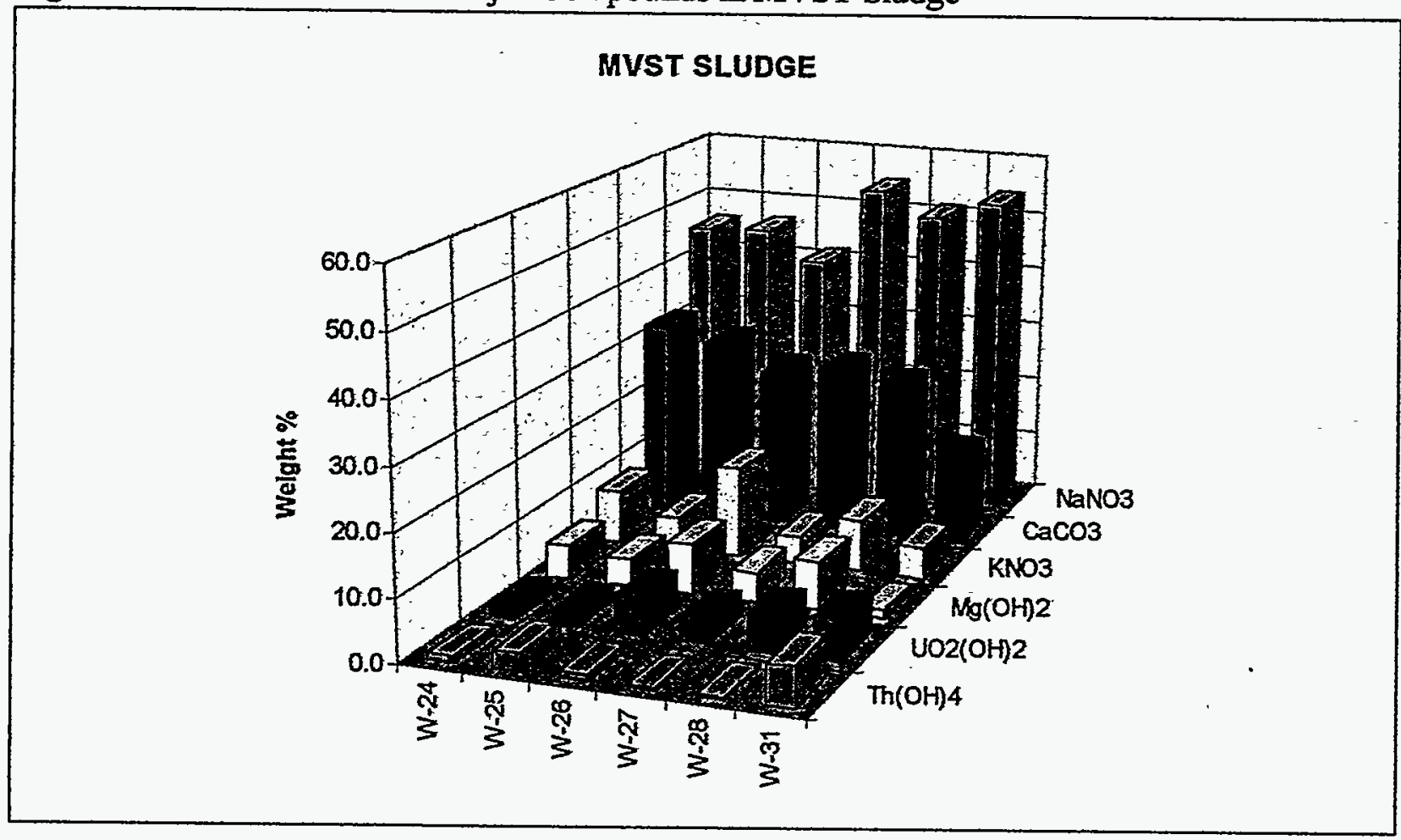

Figure 4 Distribution of Uranium and Thorium in MVST Sludge

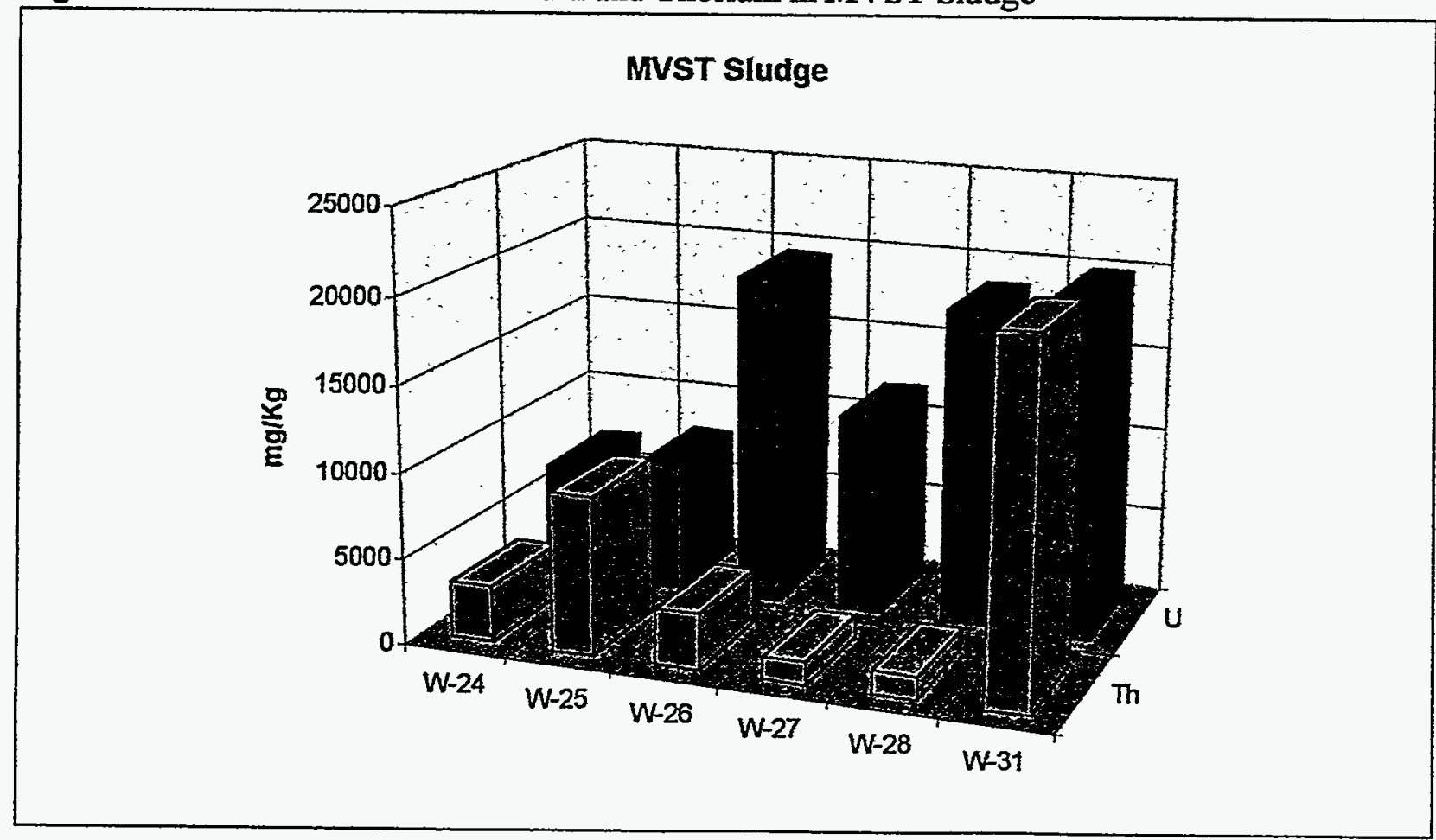


The distribution of the beta emitters found in the MVST sludge samples are summarized in Table 11. The distributions of the beta activity are shown to be dependent upon the radionuclides present, which is a function of the age of the radioactive waste, and the $\mathrm{pH}$ of the supernatant found over the sludge. Under the typical basic conditions for ORNL waste tanks, the major difference in the beta distribution between the supernatant and the sludge is that the distribution of the longer lived fission products $\left({ }^{90} \mathrm{Sr}\right.$ and $\left.{ }^{137} \mathrm{Cs}\right)$ are reversed due to the differences in solubility. The Group IA metals $\left({ }^{134} \mathrm{Cs}\right.$ and $\left.{ }^{137} \mathrm{Cs}\right)$ and the radionuclides that form anionic species $\left({ }^{99} \mathrm{TcO}_{4}^{-},{ }^{129} \mathrm{~T}\right.$, and $\left.{ }^{129} \mathrm{IO}_{3}{ }^{-}\right)$are more soluble in the supernatant. The solubility of the Group IIA metals $\left({ }^{90} \mathrm{Sr}\right)$ in the supernatant are a function of both $\mathrm{pH}$ and carbonate concentration. At high $\mathrm{pH}$ most of the other metals, lanthanides, and actinide elements form insoluble hydroxides and mixed oxides, which are found in the sludge. The ${ }^{99} \mathrm{Tc}$ activity is higher in the supernatant than the sludge. The source of most of the ${ }^{99} \mathrm{Tc}$ found in the sludge samples was the interstitial liquid, and not insoluble forms of technetium. The shorter lived radionuclides observed include the europium $\left({ }^{152} \mathrm{Eu},{ }^{154} \mathrm{Eu}\right.$, and $\left.{ }^{155} \mathrm{Eu}\right)$ isotopes and to some extent ${ }^{134} \mathrm{Cs}$.

\section{Table 11 Distribution of Beta Activity in MVST Sludge}

\begin{tabular}{|c|c|c|c|c|c|c|c|}
\hline \multirow[b]{2}{*}{ Tank } & \multirow[b]{2}{*}{ pH } & \multicolumn{6}{|c|}{ Percent of Total Beta Activity } \\
\hline & & $\begin{array}{c}{ }^{90} \mathrm{Sr} r^{\beta 0} \mathrm{Y} \\
(\%)\end{array}$ & $\begin{array}{c}{ }^{134} \mathrm{Cs}+{ }^{137} \mathrm{Cs} \\
(\%)\end{array}$ & $\begin{array}{l}{ }^{60} \mathrm{Co} \\
(\%)\end{array}$ & $\begin{array}{l}{ }^{99} \mathrm{Tc} \\
\text { (\%) }\end{array}$ & $\begin{array}{c}{ }^{152,154,155} \mathbf{E u} \\
(\%)\end{array}$ & $\begin{array}{l}{ }^{241} \mathrm{Pu} \\
(\%)\end{array}$ \\
\hline W-24 & 12.8 & 77.3 & 17.4 & 0.8 & $<0.1$ & 3.9 & 0.4 \\
\hline W-25 & 12.6 & 89.6 & 7.8 & 0.4 & $<0.1$ & 1.7 & 0.4 \\
\hline$W-26$ & 9.7 & 39.6 & 29.3 & 1.6 & $<0.1$ & 28.8 & 0.4 \\
\hline W-27 & 12.3 & 62.7 & 31.7 & 0.8 & $<0.1$ & 3.9 & 0.5 \\
\hline W-28 & 12.3 & 47.3 & 12.2 & 1.4 & $<0.1$ & 38.3 & 0.4 \\
\hline W-31 & 9.9 & 97.2 & 2.2 & 0.1 & $<0.1$ & 0.2 & 0.1 \\
\hline
\end{tabular}


Table 12 Summary of Actinide Elements in MVST Sludge

\begin{tabular}{||c|r|r|r|r|r|r||}
\hline \multirow{2}{*}{ Actinide } & \multicolumn{1}{|c|}{ W-24 } & \multicolumn{1}{|c|}{ W-25 } & W-26 & W-27 & W-28 & W-31 \\
\cline { 2 - 7 } & \multicolumn{1}{c|}{$(\% \alpha)$} & $(\% \alpha)$ & $(\% \alpha)$ & $(\% \alpha)$ & $(\% \alpha)$ & $(\% \alpha)$ \\
\hline \hline${ }^{232} \mathrm{Th}$ & 0.04 & 0.05 & 0.03 & 0.02 & 0.01 & 0.06 \\
${ }^{233} \mathrm{U}$ & 4.68 & 3.36 & 20.12 & 4.11 & 13.20 & 3.51 \\
${ }^{234} \mathrm{U}$ & 0.23 & 0.12 & 0.36 & 0.22 & 0.33 & 0.21 \\
${ }^{235} \mathrm{U}$ & $<0.01$ & $<0.01$ & $<0.01$ & 0.01 & 0.01 & $<0.01$ \\
${ }^{238} \mathrm{U}$ & $<0.01$ & $<0.01$ & $<0.01$ & $<0.01$ & $<0.01$ & $<0.01$ \\
${ }^{237} \mathrm{~Np}$ & 0.03 & 0.01 & $<0.01$ & 0.05 & 0.04 & 0.01 \\
${ }^{238} \mathrm{Pu}$ & 11.12 & 9.37 & 10.87 & 9.87 & 7.62 & 8.77 \\
${ }^{239} \mathrm{Pu}$ & 5.56 & 4.08 & 2.62 & 2.76 & 2.11 & 2.29 \\
${ }^{240} \mathrm{Pu}$ & 2.55 & 2.16 & 1.79 & 1.52 & 1.52 & 1.48 \\
${ }^{241} \mathrm{Am}$ & 11.41 & 11.17 & 7.85 & 11.52 & 11.68 & 9.45 \\
${ }^{244} \mathrm{Cm}$ & 64.38 & 69.67 & 56.35 & 69.92 & 63.47 & 74.21 \\
\hline \hline Gross $\alpha(\mathrm{Bq} / \mathrm{g})$ & 34000 & 83000 & 52000 & 26000 & 44000 & 160000 \\
\hline
\end{tabular}

${ }^{a}$ The ${ }^{241} \mathrm{Am}$ data is based on subtracting the ${ }^{238} \mathrm{Pu}$ by TIMS from the alpha peak measured at 5.15 $\mathrm{MeV}\left({ }^{238} \mathrm{Pu}+{ }^{241} \mathrm{Am}\right)$ in the alpha spectrum.

The distribution of the alpha activity is summarized in Table 12, which includes the percent alpha for each MVST sludge sample. In general, the alpha activity in the MVST system is strongly weighted by the ${ }^{244} \mathrm{Cm}$, which has a high specific activity. The list of actinides in Table 12 required several radiochemical and inorganic analytical measurements to generate the best estimates for each of the alpha activities. The ${ }^{232}$ Th activity is calculated from the total thorium measured by ICP-AES. The other thorium isotopes ${ }^{228} \mathrm{Th},{ }^{229} \mathrm{Th}$, and $\left.{ }^{230} \mathrm{Th}\right)$ are present in the ORNL sludge waste at such low mass, their presence would not effect the ICP-AES measurement. The uranium isotopes are measured by TIMS. The atom $\%$ results are converted to weight $\%$, which is used to calculate the concentration of each uranium isotope from the total uranium results obtained by ICP-AES. The activity for each uranium radionuclide is then calculated from the specific activity for each isotope. The plutonium isotopes are first measured by TIMS, and the total plutonium alpha activity, measured after a chemical separation, is used to calculate the activity for each isotope. The ${ }^{244} \mathrm{Cm}$ was measured directly by alpha spectrometry without any chemical separation. The ${ }^{241} \mathrm{Am}$ activity is 
determined by subtracting the ${ }^{238} \mathrm{Pu}$ activity from the sum of the ${ }^{238} \mathrm{Pu}+{ }^{241} \mathrm{Am}$ measured by alpha spectrometry. Both ${ }^{238} \mathrm{Pu}$ and ${ }^{241} \mathrm{Am}$ have an alpha energy of about $5.50 \mathrm{MeV}$ and can not be resolved by alpha spectrometry. There was no chemical separation of the plutonium and americium for this project because of cost concerns.

\subsection{RCRA Characteristics for the MVST System}

The RCRA regulatory limits are listed in Table 13, which also includes the limits for the EPA Toxicity Characteristic Leaching Protocol (TCLP) extract and the functional total metal limits for a solid or sludge waste. The total metal limits are a factor of twenty times higher than the TCLP extraction limits and are based on the 1:20 dilution used for the TCLP extraction procedure.

\section{Table 13 Summary of RCRA Regulatory Limits}

\begin{tabular}{|llccc||}
\hline Metals & & $\begin{array}{c}\text { TCLP Extract } \\
\text { and Liquids } \\
\text { (mg/L) }\end{array}$ & $\begin{array}{c}\text { Solid/Sludge } \\
\text { Total Metal } \\
\text { (mg/Kg) }\end{array}$ \\
\hline \hline Silver & $(\mathrm{Ag})$ & 5 & 100 \\
Arsenic & $(\mathrm{As})$ & 5 & 100 \\
Barium & $(\mathrm{Ba})$ & 100 & 2000 \\
Cadmium & $(\mathrm{Cd})$ & 1 & 20 \\
Chromium & $(\mathrm{Cr})$ & 5 & 100 \\
Mercury & $(\mathrm{Hg})$ & 0.2 & 4 \\
Nickel & $(\mathrm{Ni})$ & 50 & 1000 \\
Lead & $(\mathrm{Pb})$ & 5 & 100 \\
Selenium & $(\mathrm{Se})$ & 1 & 20 \\
Thallium & $(\mathrm{Tl})$ & 0.9 & 18 \\
\hline
\end{tabular}

If the RCRA metal concentrations are found to be below the total metal limits, the solid waste can not fail the TCLP leach test. If the RCRA metal concentrations exceed the total metal limits, the TCLP leach test must be done to determine if the solid waste is hazardous. For solid samples, the 
TCLP leach test is only valid for the final waste form ready for disposal. The total metal concentration data can be used as acceptable process knowledge if the final waste form only results in a dilution of the RCRA metal concentrations. Examples of waste forms that result in a dilution of a solid waste includes grouting ( 2 fold dilution) and vitrification ( 3 fold dilution). If the total metal limit is exceeded after stabilizing the waste, the TCLP leach test would be required for only the metals that had the potential to exceed the regulatory limits.

Several of the supernatant samples from the MVST tanks exceed the RCRA regulatory limits. Waste tanks W-26, W-27, W-28, and W-31 slightly exceed the limit for mercury. Waste tanks W-24, W25 , W-26, and W-31 come close to exceeding the limits for cadmium and could be considered over the limit depending on the confidence limits used. The current technology used for long term storage of the liquid waste is a solidification process that results in a final waste form that passes the TCLP leach test. The nickel and thallium are proposed RCRA metals and are included in the data for future waste management decisions.

All of the MVST tank sludge samples exceed the total metal limits for lead and mercury, and two tanks are over or near the limit for chromium. Most of the ORNL radioactive waste sludge samples, characterized to date, have exceeded the total metal limits for these three RCRA metals. Based on past experience, it is expected that solidification of the ORNL MVST sludge would fix these RCRA metals such that the final waste form would pass the TCLP leach test.

\subsection{TRU Classifications for LLLW System}

The DOE definition for Transuranic (TRU) Waste includes the following conditions,

- TRU activity $\geq 3700 \mathrm{~Bq} / \mathrm{g}(100 \mathrm{nCi} / \mathrm{g})$,

- TRU isotopes must be alpha emitting actinide with $Z>92$ (uranium),

- TRU isotopes must have a half life $\geq 20$ years.

This definition excludes all thorium and uranium isotopes. The short lived actinide ${ }^{244} \mathrm{Cm}\left(\mathrm{t}_{1 / 2}=18.1\right.$ years), which is common to ORNL waste, falls outside the TRU definition. Also, the plutonium isotope, ${ }^{241} \mathrm{Pu}$, would be excluded from calculation of the TRU activity because it is a pure beta 
emitter. The primary actinide elements common to ORNL waste, that are present at sufficient levels to meet the TRU definition, include ${ }^{238} \mathrm{Pu},{ }^{239} \mathrm{Pu},{ }^{240} \mathrm{Pu}$, and ${ }^{241} \mathrm{Am}$. There is some current work at the Radiochemical Engineering Development Center (Mark-42 fuel assembly processing) that could generate enough ${ }^{243} \mathrm{Am}$ to make a significant contribution to TRU alpha content of the waste. The remaining actinide elements present in ORNL waste are generally not available at high enough activity, and/or do not have a long enough half-life to meet the TRU definition.

None of the MVST supernatant samples discussed in this report had enough alpha activity to be considered as TRU waste. All of the MVST sludge that has been characterized to date has been classified as TRU waste based on only the plutonium and americium activity. The alpha activity reported is based on wet weight, if adjusted for dry weight the activity would almost double. The MVST sludge samples contained enough plutonium and americium activity to easily satisfy the WIPP waste acceptance criteria ${ }^{12}$ for transuranic waste. Based on the TRU activity, any dilution of the sludge that would result from a solidification process such as grouting or vitrification would most likely not effect the TRU classification.

\subsection{Distribution of Fissile Material in LLLW System}

As discussed in section 3.5, the ORNL LLLW waste acceptance criteria (WAC) requires the fissile isotopes of uranium and plutonium to be diluted with ${ }^{238} \mathrm{U}$ and ${ }^{232} \mathrm{Th}$, respectively. Table 14 summarizes the dilution or "denature" ratios for the MVST supernatant samples. All the dilution ratios for the MVST liquid phase exceed the required dilution factors. Only one of the supernatant samples, W-31, had enough thorium and plutonium to allow estimates for the plutonium dilution ratios. A summary of the dilution ratios for fissile material in the sludge samples is provided in Table 15. All the dilution ratios for the MVST sludge samples exceed the required dilution factors for the fissile isotopes of uranium and plutonium. All the dilution ratios listed in Table 14 and 15 are based on equations discussed in section 3.5 of this report. 
Table 14 Summary of Denature Ratios for MVST Supernatant

\begin{tabular}{||c|c|c|c|c|c||}
\hline Tank & $\begin{array}{c}{ }^{238} \mathbf{U}{ }^{235} \mathbf{U} f_{35} \\
\text { (eq. 1) }\end{array}$ & $\begin{array}{c}{ }^{238} \mathbf{U} \text { (35 }^{35} \\
\text { (eq. 3) }\end{array}$ & $\begin{array}{c}{ }^{238} \mathbf{U}^{{ }^{33} \mathbf{U}} \\
\text { (eq. 4) }\end{array}$ & $\begin{array}{c}{ }^{232} \mathbf{T h}{ }^{239} \mathbf{P u} \\
\text { (eq. 2) }\end{array}$ & pH \\
\hline \hline W-24 & 231 & 273 & 632 & na $^{\mathrm{a}}$ & 12.3 \\
W-25 & 203 & 226 & 571 & na & 12.6 \\
W-26 & 225 & 281 & 521 & na & 8.4 \\
W-27 & 256 & 312 & 729 & na & 12.8 \\
W-28 & 252 & 321 & 626 & na & 7.3 \\
W-31 & 226 & 263 & 622 & 184 & 10.0 \\
\hline
\end{tabular}

${ }^{2}$ Concentration of thorium and plutonium to low to calculate ratio.

Table 15 Summary of Denature Ratios for MVST Sludge

\begin{tabular}{|c|c|c|c|c|c||}
\hline Tank & $\begin{array}{c}{ }^{238} \mathbf{U}{ }^{235} \mathbf{U} f_{35} \\
\text { (eq. 1) }\end{array}$ & $\begin{array}{c}{ }^{238} \mathbf{U} /{ }^{335} \mathbf{U} \\
\text { (eq. 3) }\end{array}$ & $\begin{array}{c}{ }^{238} \mathbf{U}{ }^{233} \mathbf{U} \\
\text { (eq. 4) }\end{array}$ & $\begin{array}{c}{ }^{232} \mathbf{T h}{ }^{239} \mathbf{P u} \\
\text { (eq. 2) }\end{array}$ & pH \\
\hline \hline W-24 & 177 & 181 & 862 & 3920 & 12.8 \\
W-25 & 153 & 153 & 470 & 6320 & 12.6 \\
W-26 & 227 & 279 & 545 & 5730 & 9.7 \\
W-27 & 298 & 313 & 3070 & 4390 & 12.3 \\
W-28 & 296 & 347 & 1120 & 3750 & 12.3 \\
W-31 & 145 & 144 & 581 & 13900 & 9.9 \\
\hline
\end{tabular}


The dilution ratios listed in Tables 14 and 15 are base on the ratio of weight $\%$, not the ratio of atom $\%$ given in the data tables. There is a small difference between atom $\%$, reported for the uranium and plutonium, and weight $\%$, which is needed for many calculations performed with the analytical data. To convert from atom $\%$ to weight $\%$, we used the following equation,

$$
\begin{aligned}
& W_{i}=\frac{a_{i} M_{i}}{\sum_{i}^{n} a_{i} M_{i}} X 100 \% \\
& \text { where, } \quad \mathrm{W}_{\mathrm{i}} \quad=\text { weight } \% \text {, } \\
& \mathrm{M}_{\mathrm{i}} \quad=\text { nuclidic mass } \\
& \mathrm{a}_{\mathrm{i}} \quad=\text { atom } \% \text {. }
\end{aligned}
$$

An example of this calculation is provided in Table 16, which shows there is not much difference between the atom $\%$ and the weight $\%$.

Table 16 Example of Converting Atom \% to Weight \% for W-31 Sludge

\begin{tabular}{|c|c|c|r|r||}
\hline Isotope & $\begin{array}{c}\text { Nuclidic mass } \\
(\mathbf{g} / \mathbf{m o l})\end{array}$ & atom \% & $\left.\mathbf{( a}_{\mathbf{i}} \mathbf{M}_{\mathbf{i}}\right)$ & weight $\%$ \\
\hline \hline${ }^{233} \mathrm{U}$ & 233.039629 & 0.056 & 13.0502 & 0.0548 \\
${ }^{234} \mathrm{U}$ & 234.040947 & 0.004 & 0.9362 & 0.0039 \\
${ }^{235} \mathrm{U}$ & 235.043924 & 0.621 & 145.9623 & 0.6132 \\
${ }^{236} \mathrm{U}$ & 236.045563 & 0.002 & 0.4721 & 0.0020 \\
${ }^{238} \mathrm{U}$ & 238.050785 & 99.316 & 23642.2518 & 99.3260 \\
& & 99.999 & 23802.6726 & 99.9999 \\
\hline
\end{tabular}

The distribution of plutonium isotopes by alpha activity are illustrated in Fig. 5 for each of the MVST samples. For comparison, Fig. 6 shows the distribution of the plutonium isotopes by concentration for each of the MVST sludge samples. One should note that the ${ }^{238} \mathrm{Pu}$ dominates the alpha activity and the ${ }^{239} \mathrm{Pu}$ is the major isotope by weight or concentration. 
Figure 5 Distribution of Plutonium by Alpha Activity in MVST Sludge

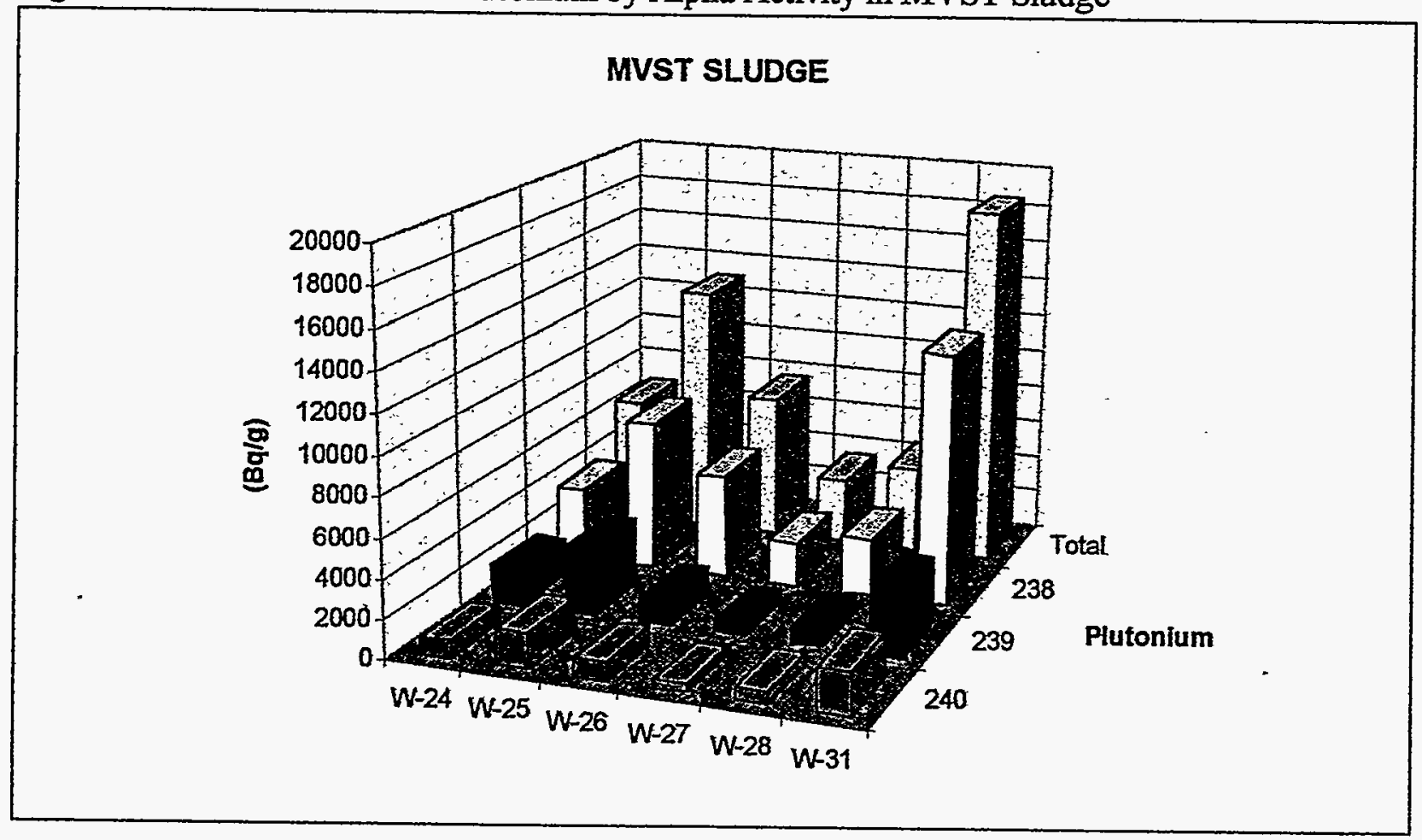

Figure 6 Distribution of Plutonium by Concentration in MVST Sludge

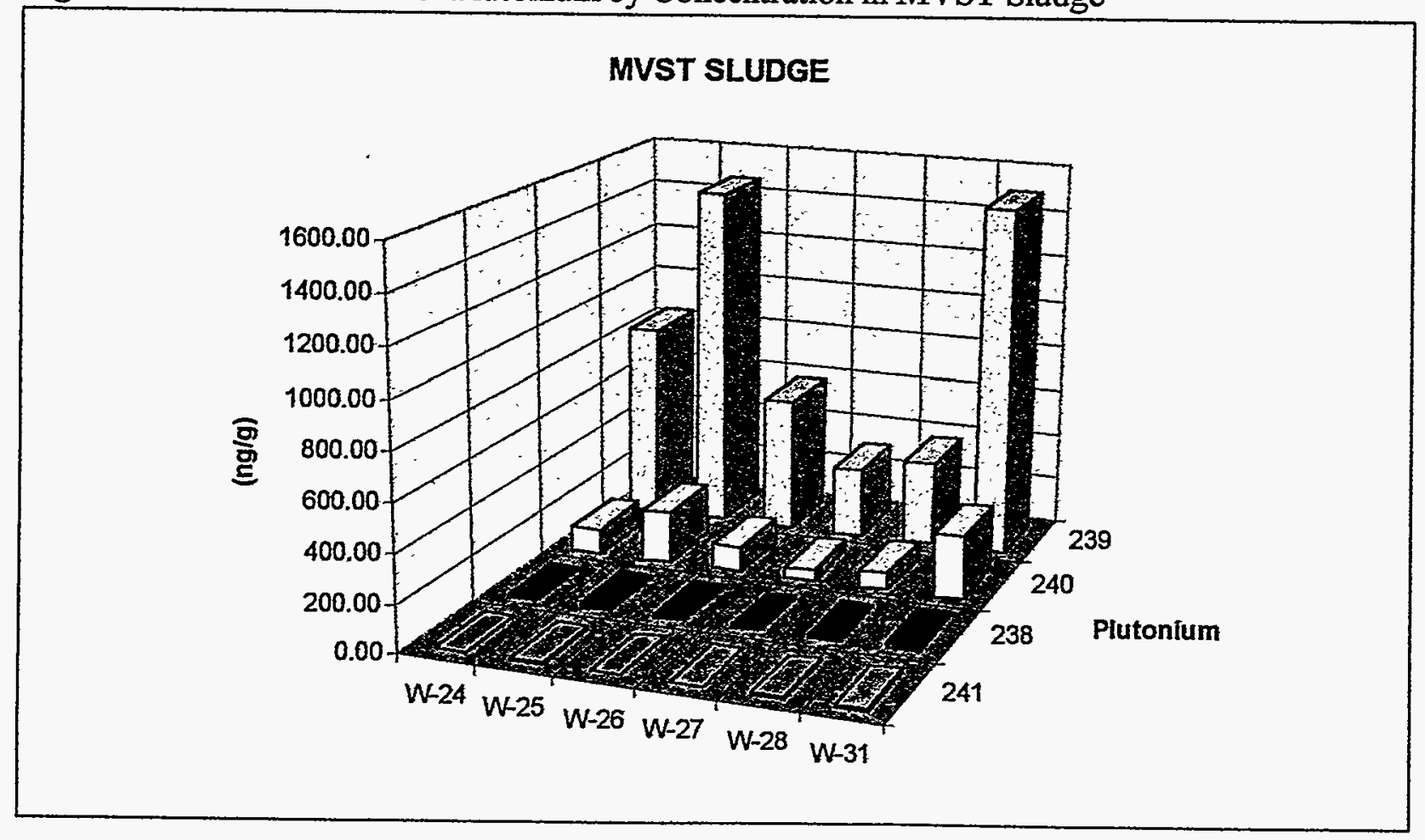





\subsection{Discussion of the Total Anion Content in the Sludge}

As discussed in section 3.3, there were three sample preparation methods used to investigate the total anion content of the sludge samples, which included (1) water leach, (2) oxygen bomb combustion, and (3) sodium peroxide/sodium hydroxide fusion. A summary and comparison of these sludge preparation methods are given in Table 17.

\section{Table 17 Summary of Total Anion Data for MVST Sludge}

\begin{tabular}{|c|c|c|c|c|c|c|c|}
\hline \multirow[t]{2}{*}{ Anion } & \multirow[t]{2}{*}{ Method } & \multicolumn{6}{|c|}{ (mg/Kg) } \\
\hline & & $W-24$ & W-25 & W-26 & W-27 & W-28 & W-31 \\
\hline Bromide & $\begin{array}{l}\text { Water Leach' } \\
\text { Bomb } \\
\text { Fusion }\end{array}$ & $\begin{array}{l}<47 \\
213 \\
320\end{array}$ & $\begin{array}{r}<49 \\
158 \\
236\end{array}$ & $\begin{array}{r}<50 \\
322 \\
420\end{array}$ & $\begin{array}{r}<48 \\
117 \\
290\end{array}$ & $\begin{array}{l}<48 \\
133 \\
287\end{array}$ & $\begin{array}{l}<45 \\
118 \\
240\end{array}$ \\
\hline Chloride & $\begin{array}{l}\text { Water Leach } \\
\text { Bomb } \\
\text { Fusion }\end{array}$ & $\begin{array}{r}2770 \\
2740 \\
n d^{2}\end{array}$ & $\begin{array}{r}2110 \\
2170 \\
n d^{2}\end{array}$ & $\begin{array}{r}3070 \\
2800 \\
\text { nd }^{2}\end{array}$ & $\begin{array}{r}2280 \\
1850 \\
\mathrm{nd}^{2}\end{array}$ & $\begin{array}{r}3460 \\
3210 \\
\text { nd }^{2}\end{array}$ & $\begin{array}{r}2570 \\
2150 \\
n^{2}\end{array}$ \\
\hline Fluoride & $\begin{array}{l}\text { Water Leach } \\
\text { Bomb } \\
\text { Fusion }\end{array}$ & $\begin{array}{r}103 \\
41 \\
n d^{2}\end{array}$ & $\begin{array}{r}118 \\
71 \\
n d^{2}\end{array}$ & $\begin{array}{r}<50 \\
81 \\
n d^{2}\end{array}$ & $\begin{array}{r}<48 \\
44 \\
\mathrm{nd}^{2}\end{array}$ & $\begin{array}{r}<48 \\
64 \\
n d^{2}\end{array}$ & $\begin{array}{l}125 \\
218 \\
n^{2}\end{array}$ \\
\hline Nitrate & $\begin{array}{l}\text { Water Leach } \\
\text { Bomb } \\
\text { Fusion }\end{array}$ & $\begin{array}{r}165000 \\
111000 \\
90400\end{array}$ & $\begin{array}{r}162000 \\
101000 \\
71800\end{array}$ & $\begin{array}{l}214000 \\
148000 \\
113000\end{array}$ & $\begin{array}{l}210000 \\
120000 \\
143000\end{array}$ & $\begin{array}{l}248000 \\
167000 \\
204000\end{array}$ & $\begin{array}{l}197000 \\
119000 \\
157000\end{array}$ \\
\hline Nitrite & $\begin{array}{l}\text { Water Leach } \\
\text { Bomb } \\
\text { Fusion }\end{array}$ & $\begin{array}{r}2250 \\
7310 \\
32400\end{array}$ & $\begin{array}{r}4970 \\
8090 \\
39000\end{array}$ & $\begin{array}{r}1650 \\
5820 \\
38000\end{array}$ & $\begin{array}{r}2280 \\
6090 \\
38400\end{array}$ & $\begin{array}{r}1120 \\
8090 \\
11600\end{array}$ & $\begin{array}{r}3470 \\
6190 \\
10300\end{array}$ \\
\hline Phosphate & $\begin{array}{l}\text { Water Leach } \\
\text { Bomb } \\
\text { Fusion } \\
\text { ICP-AES } \\
\text { ICP-MS }\end{array}$ & $\begin{array}{r}<19 \\
114 \\
277 \\
3800 \\
-\end{array}$ & $\begin{array}{r}<20 \\
<51 \\
340 \\
5670 \\
4350\end{array}$ & $\begin{array}{r}<20 \\
89 \\
211 \\
3280 \\
-\end{array}$ & $\begin{array}{r}<19 \\
<36 \\
<190 \\
3070 \\
-\end{array}$ & $\begin{array}{r}<19 \\
117 \\
<190 \\
2780 \\
-\end{array}$ & $\begin{array}{r}<45 \\
253 \\
1540 \\
13000 \\
9430\end{array}$ \\
\hline Sulfate & $\begin{array}{l}\text { Water Leach } \\
\text { Bomb } \\
\text { Fusion } \\
\text { ICP-MS }\end{array}$ & $\begin{array}{r}1370 \\
1520 \\
2540 \\
- \\
\end{array}$ & $\begin{array}{r}1750 \\
2070 \\
2950 \\
29300\end{array}$ & $\begin{array}{r}2120 \\
2460 \\
4600 \\
-\end{array}$ & $\begin{array}{r}549 \\
752 \\
4270 \\
-\end{array}$ & $\begin{array}{r}1770 \\
1810 \\
3260 \\
-\end{array}$ & $\begin{array}{l}1090 \\
1030 \\
1650 \\
9500\end{array}$ \\
\hline
\end{tabular}

${ }^{1}$ Unable to resolve bromide peak from nitrate on ion chromatography column used for this sample.

${ }^{2}$ Unable to quantify by ion chromatography due to interference from fusion matrix.

\subsubsection{Nitrate/Nitrite}

It is difficult to compare the yield for these two anions between the three preparation methods. The majority of the compounds present in the MVST waste system that contain nitrate and nitrite readily dissolve in water and are accounted for in the water leaches. This can be argued by looking at the 
cation/anion charge balance calculations for the sludge analysis. These calculations show acceptable agreement between the anionic species and the cationic species (which are accurately determined by conventional methods) present in the sludges. The majority of the anion contribution is by far due to the nitrate ion with the other anions contributing just a fraction of the total negative molar charge. Based on this calculated charge balance it is believed that the majority of the nitrates are accounted for in the water leaches. When the sludge is prepared using either the bomb or fusion method the sample is subject to an oxidizing environment which will not only change the nitrate/nitrite ratio in the sample but will also oxidize any nitrogen present in the sample to nitrate or nitrite. The ratio of the nitrate/nitrite measured after the bomb or fusion methods does not represent the ratio in the original sample. The mole percent of nitrite relative to the sum of the nitrate and nitrite ranged from $0.6 \%$ to $4 \%$ in the water leach samples and represents the nitrite content of the samples as recieved. The mole percent of nitrite observed after the bomb combustion ranged from $5 \%$ to $9.7 \%$, and after the fusion preparation ranged from $7.1 \%$ to $42 \%$. The change in the mole percent of nitrite is a function of the oxidizing environment from each preparation method.

\subsubsection{Halides (fluoride, chloride, bromide)}

The data in Table 17 shows that there is no benefit for using a bomb combustion over a water leach of the MVST sludges for fluoride and chloride. For the bromide results the water leach was analyzed using a Dionex AS4A ion exchange column while the bomb combustion and fusion results were analyzed using a new Dionex AS14 ion exchange column. The older AS4A column separation of the bromide and nitrate peaks is not as good as the AS14 and due to the high levels of nitrate present in the sludges the bromide peak could not be resolved from the nitrate peak using the AS4A column. Therefore, a comparison could not be made between the water leaches and the other methods for bromide. It does appear that the fusion method yields slightly higher bromide values than the bomb.

Due to the extremely high levels of sodium in the sample matrix after the fusion it was impossible to determine fluoride or chloride concentrations using ion chromatography. The matrix caused large interfering peaks to elute off of the column at the beginning of the analysis run where fluoride is detected; then a large negative dip occurred in the chromatogram where chloride is eluted. It may 
be possible to measure fluoride and chloride in the fusion matrix after using a clean-up procedure prior to analysis. Possible solutions are being investigated.

\subsubsection{Phosphate}

It is believed that a large fraction of the phosphate in the MVST sludge is present as tributyl phosphate and degradation products dibutyl-and monobutyl phosphate. The tributyl phosphate has low solubility in water and would not be seen in the water leach. This is illustrated in the table showing phosphate values below the detection limit of the instrument. When the sludges were prepared using the Parr bomb some phosphate was detected but still at low levels. The explanation for this could be due to poor combustion of the sludge. In order to obtain an adequate combustion 5000 calories of heat must be produced in the bomb. Since the MVST sludges are not comprised of combustible material all of the heat must be generated by the combustion aid (mineral oil). Using the heat of combustion for mineral oil, $0.5 \mathrm{~mL}$ was determined to be able to produce greater than 5000 calories and therefore provide for an adequate combustion. After a material undergoes complete combustion it should have an ash like appearance. By visual observation after the sludge was combusted it appears to be just dried out sludge with a crusty appearance. Based on this and the fact that only low levels of phosphates were detected it is felt that the bomb is a poor choice for preparation of the sludge for anion determination. A good sample for bomb combustion would have some combustibility with the combustion aid acting as a catalyst to start the reaction. The MVST sludges have no combustible properties.

When phosphate was determined using the fusion method slightly higher values were obtain. However, four matrix spiked samples were analyzed with the fusion batches and all spike recoveries were zero percent. This indicates that phosphate is lost during the fusion preparation and therefore the method used as is, is not adequate for the analysis of phosphate in the sludges.

Also shown in the Table 17 are the phosphate results by ICP-AES for each MVST sludge sample and two results by ICP-MS for W-25 and W-31. These phosphate values are calculated results based on the analysis of total phosphorus by the ICP methods after a closed vessel microwave acid digestion of the sludge. The ICP-AES values are currently considered to be the best results for the 
total phosphorus in the sludge. The ICP-MS measurements are estimates done to confirm the high levels of phosphorus observed in the MVST sample. The water leach, bomb combustion, and fusion methods all yielded phosphate results much lower than the ICP measurements after an acid digestion.

\subsubsection{Sulfate}

The analysis of sulfate between the water leaches and the bomb combustion show good agreement but there may be sulfate compounds present in the sludge that are not water soluble and would not be accounted for using the bomb procedure due to the problems discussed earlier. The fusion results show an appreciable increase in the measured sulfate concentration. But one needs to keep in mind that this is a total sulfur determination and the sulfate measured does not necessarily have to come from a sulfate compounds. Similar to the phosphate, sulfate is lost during the fusion preparation phase. Four matrix spikes analyzed with the fusion batch averaged out to only a thirty percent spike recovery. Two cursory measurements for sulfur by ICP-MS on the W-25 and W-31 sludge samples produced much higher sulfate equivalent values than the other sample preparation methods. The ICP-MS technique for sulfur needs additional investigation to ensure molecular mass interference problems are properly accounted for with the sludge samples. This investigation would have been out of scope for this project.

\subsubsection{Summary}

There is no ideal method to obtain a total anion content on the MVST sludges. The water leaches are considered to be adequate for nitrate; nitrite, and the halides. The total phosphate and sulfate content however will not be obtained by a water leach and any method used that oxidizes the sample would be considered to be a total phosphorus or sulfur. The best preparation method for total phosphorus or sulfur appears to be closed vessel microwave digestion followed by analysis by ICPAES or ICP-MS. Other DOE sites that have experience with caustic high nitrate sludge samples and have worked with the bomb and fusion procedures, have related similar observations which include poor yields and heavy matrix interferences associated with these preparation methods. 


\subsection{Solubility of MVST Sludge in Water}

The MVST sludge samples were taken through a water wash to determine the water soluble anions and measure the effect of the sludge on $\mathrm{pH}$. Since this water leach solution was available, several of the lower cost analytical measurements, including the metals by ICP-AES, gross alpha/beta, and gamma emitters, were measured on the wash solution to evaluate the relative solubility of the sludge in water. The water wash experiment consisted of taking 5 grams of wet sludge and diluting the sample to $50 \mathrm{~mL}$ with deionized water. The sludge was leached with the water on a vortex mixer for several minutes and the clarified liquid was then removed for analysis. Results from the water leaching experiment are summarized in Table 18.

Table 18 Recovery of Selected Species in Water Leach

\begin{tabular}{|c|c|c|c|c|c|c|}
\hline \multirow{2}{*}{$\begin{array}{l}\text { Analytical } \\
\text { Measurement }\end{array}$} & \multicolumn{6}{|c|}{ \% Recovery in Water Leach of Sludge } \\
\hline & W-24 & W-25 & W-26 & W-27 & W-28. & W-31 \\
\hline \multicolumn{7}{|l|}{ Selected metals } \\
\hline $\mathrm{pH}$ of water wash & 12.8 & 12.6 & 9.7 & 12.3 & 12.3 & 9.9 \\
\hline $\mathrm{Al}$ & 14.75 & 2.82 & $<0.01$ & 0.80 & $<0.01$ & 0.06 \\
\hline $\mathrm{Ca}$ & 1.06 & 0.95 & 3.07 & 14.49 & 32.75 & 2.29 \\
\hline $\mathrm{Cr}$ & 7.47 & 33.55 & 1.53 & 8.3 & 0.91 & 12.46 \\
\hline $\mathrm{Fe}$ & $<0.01$ & $<0.01$ & $<0.01$ & $<0.01$ & $<0.01$ & $<0.01$ \\
\hline $\mathrm{K}$ & 102.99 & 108.59 & 103.56 & 114.78 & 105.48 & 103.49 \\
\hline $\mathrm{Mg}$ & 0.01 & 0.01 & 5.51 & $<0.01$ & $<0.01$ & 0.38 \\
\hline $\mathrm{Na}$ & 105.94 & 102.69 & 110.02 & 107.22 & 111.80 & 106.44 \\
\hline Th & $<0.01$ & $<0.01$ & 0.02 & $<0.01$ & $<0.01$ & $<0.01$ \\
\hline $\mathrm{U}$ & 0.04 & 0.04 & 0.03 & $<0.01$ & $<0.01$ & 1.15 \\
\hline \multicolumn{7}{|c|}{ Selected radionnclides } \\
\hline Gross alpha & 0.38 & 0.13 & 0.18 & 0.12 & 0.07 & $<0.01$ \\
\hline Gross beta & 10.44 & 2.29 & 28.57 & 30.63 & 16.77 & 1.33 \\
\hline 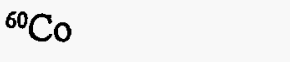 & 0.27 & 0.24 & 2.07 & 1.00 & 0.95 & 0.50 \\
\hline 列 $\mathrm{Cs}$ & 71.70 & 31.92 & 96.63 & 84.62 & 93.55 & 48.84 \\
\hline${ }^{152} \mathrm{Eu}$ & $<0.01$ & $<0.01$ & 0.09 & $<0.01$ & 0.14 & $<0.01$ \\
\hline
\end{tabular}


The water leach appears to remove more of the sodium and potassium than the total digestion with nitric acid, however, the high recovery is more likely due to the difference in sample size used for each sample preparation. Only $0.5 \mathrm{~g}$ of sample was used for the nitric acid digestion as compared to the $5 \mathrm{~g}$ used for the water leach. The larger sample size would be more representative of the overall sludge and introduces less sampling error. The water wash does not remove many of the metals (i.e. uranium) that cause spectral interference problems. Therefore, both analytical and sampling errors most likely contribute to the high bias for the sodium and potassium recovery. In general, the lighter alkali metals are quantitatively removed from the sludge along with the nitrate. Some of the cesium (see ${ }^{137} \mathrm{Cs}$ recovery) appears to be bound to the sludge, which could be due to differences in ion exchange properties between the cesium and the lighter alkali metals. The incomplete removal of ${ }^{137} \mathrm{Cs}$ from the sludge with water, caustic, and low acid washing has been observed in past experiments with the MVST sludge.

As expected, the actinide and lanthanide elements are not significantly removed by the water wash and this behavior is illustrated by the uranium, thorium, ${ }^{152} \mathrm{Eu}$, and gross alpha recovery listed in Table 18. The water solubility of the alkaline earth elements, represented by calcium in Table 18, are a function of both the $\mathrm{pH}$ and the carbonate concentration. The recovery of the calcium ranges from $<1 \%$ to about $33 \%$ for the MVST sludge samples, and this behavior would also be expected for the ${ }^{90} \mathrm{Sr}$ activity. Most of the other major metals are usually insoluble in a water wash except for the chromium which is probably present as an the anion chromate, and many anionic species tend to be soluble in water.

\subsection{Estimates for Compliance with WIPP WAC, Rev. 5 for MVST Sludge}

The purpose of this section is to establish upper boundary estimates, based upon a 55-gal. drum shipping container, for several of the nuclear properties criteria and requirements for RH-TRU waste as specified in the WIPP WAC, Revision 5. Specifically, this section will develop estimates for the ${ }^{239} \mathrm{Pu}$ Fissile Gram Equivalent (FGE), ${ }^{239} \mathrm{Pu}$ Equivalent Activity, and Thermal Power or decay heat limits per RH-TRU canister. The RH-TRU limits per waste canister for each of these nuclear criteria are listed as follows, 


$\begin{array}{lll}- & { }^{239} \mathrm{Pu} F G E & <325 \mathrm{~g} \\ - & { }^{239} \mathrm{Pu} \text { Equivalent Activity } & <1000 \mathrm{Ci} \\ \text { - Thermal Power } & & <300 \text { watts. }\end{array}$

For the MVST sludge, the ${ }^{239} \mathrm{Pu}$ FGE can be estimated by the summation of the gram-equivalents for ${ }^{233} \mathrm{U},{ }^{235} \mathrm{U}$, and ${ }^{239} \mathrm{Pu}$. As shown in Table 19, the ${ }^{235} \mathrm{U}$ dominates the total ${ }^{239} \mathrm{Pu}$ FGE for the MVST sludge samples and the ${ }^{239} \mathrm{Pu}$ is less than $5 \%$ of the total fissile gram equivalent. Based on packaging the wet sludge in 55-gal. drums, none of the MVST sludge would approach the RH-TRU limit of $325 \mathrm{~g}$ per canister for the ${ }^{239} \mathrm{Pu}$ FGE. Estimates for the total weight (Kg) of sludge in a 55 gal. drum, for each MVST sludge sample, are listed in Table 22.

Table 19 Estimates for ${ }^{239} \mathrm{Pu}$ FGE with the MVST Sludge

\begin{tabular}{|c|c|c|c|c|c|c|c|}
\hline Isotope & $\begin{array}{c}{ }^{239} \mathrm{Pu} \text { FGE } \\
\text { factor }\end{array}$ & $\begin{array}{c}W-24 \\
\text { (mg/Kg) }\end{array}$ & $\begin{array}{c}\text { W-25 } \\
\text { (mg/Kg) }\end{array}$ & $\begin{array}{c}\text { W-26 } \\
\text { (mg/Kg) }\end{array}$ & $\begin{array}{c}W-27 \\
\text { (mg/Kg) }\end{array}$ & $\begin{array}{c}\text { W-28 } \\
\text { (mg/Kg) }\end{array}$ & $\begin{array}{c}\text { W-31 } \\
\text { (mg/Kg) }\end{array}$ \\
\hline${ }^{233} \mathrm{U}$ & 0.865 & 3.58 & 6.6 & 25.1 & 2.52 & 12.0 & 10.9 \\
\hline${ }^{235} \mathrm{U}$ & 0.641 & 33.2 & 41.0 & 51.3 & 35.7 & 46.2 & 121 \\
\hline${ }^{239} \mathrm{Pu}$ & 1.000 & 0.84 & 1.46 & 0.57 & 0.29 & 0.36 & 1.49 \\
\hline \multicolumn{2}{|c|}{${ }^{239} \mathrm{Pu} F \mathrm{FE}(\mathrm{mg} / \mathrm{Kg})$} & $25: 22$ & 3345 & 55.16 & $25: 35$ & 40.35 & 88.48 \\
\hline \multicolumn{2}{|c|}{${ }^{239} \mathrm{Pu}$ FGE in 55 gal (g) } & 722 & 95 & 15,8 & 7.6 & 11.5 & 26.5 \\
\hline
\end{tabular}

Estimates for the total ${ }^{239} \mathrm{Pu}$ equivalent activity (Ci) in a 55-gal. drum for each of the MVST sludge samples are listed in Table 20 . The ${ }^{239} \mathrm{Pu}$ equivalent activity is based on following calculation,

$$
{ }^{239} \mathrm{Pu} \text { Equivalent Activity }=\sum_{i=1} \frac{A_{i}}{F_{i}}
$$

where $A_{i}$ is the activity of radionuclide $i$, and $F_{i}$ is the ${ }^{239} \mathrm{Pu}$ equivalent activity weighting factor for radionuclide $i$. The weighting factors for the major radionuclides found in the MVST sludge are listed in Table 20. As shown in the last row of Table 20, all of the MVST sludge estimates for ${ }^{239} \mathrm{Pu}$ Equivalent activity would be less than $1 \mathrm{Ci}$ for a $55 \mathrm{gal}$. drum, which is well below the RH-TRU limits. The MVST sludge is well below the $\mathrm{CH}$-TRU limit of $80 \mathrm{Ci}$ of plutonium equivalent activity for untreated waste in a 55-gal. drum and will not approach the $1000 \mathrm{Ci}$ WAC limit for a RH-TRU canister, which holds three 55 -gal. drums. 
Table 20 Estimates for ${ }^{239} \mathrm{Pu}$ Equivalent Activity with the MVST Sludge

\begin{tabular}{|c|c|c|c|c|c|c|c|}
\hline Isotope & $\begin{array}{l}{ }^{239} \mathrm{Pu} \\
\text { wt. factor }\end{array}$ & $\begin{array}{l}W-24 \\
(\mathrm{~Bq} / \mathrm{g})\end{array}$ & $\begin{array}{l}\mathrm{W}-25 \\
(\mathrm{~Bq} / \mathrm{g})\end{array}$ & $\begin{array}{l}\text { W-26 } \\
(\mathrm{Bq} / \mathrm{g})\end{array}$ & $\begin{array}{l}\mathrm{W}-27 \\
(\mathrm{~Bq} / \mathrm{g}) \\
\end{array}$ & $\begin{array}{l}\text { W-28 } \\
(\mathrm{Bq} / \mathrm{g})\end{array}$ & $\begin{array}{l}\text { W-31 } \\
(\mathrm{Bq} / \mathrm{g})\end{array}$ \\
\hline${ }^{233} \mathrm{U}$ & 3.9 & 1600 & 2800 & 10000 & 1000 & 5200 & 5200 \\
\hline${ }^{238} \mathrm{Pu}$ & 1.1 & 3800 & 7800 & $\cdot 5400$ & 2400 & 3000 & 13000 \\
\hline${ }^{239} \mathrm{Pu}$ & 1.0 & 1900 & 3400 & 1300 & 670 & 830 & 3400 \\
\hline${ }^{240} \mathrm{Pu}$ & 1.0 & 870 & 1800 & 890 & 370 & 600 & 2200 \\
\hline${ }^{241} \mathrm{Pu}$ & 52.0 & 14000 & 26000 & 15000 & 6500 & 12000 & 24000 \\
\hline${ }^{241} \mathrm{Am}$ & 1.0 & 3900 & 9300 & 3900 & 2800 & 4600 & 14000 \\
\hline${ }^{244} \mathrm{Cm}$ & 1.9 & 22000 & 58000 & 28000 & 17000 & 25000 & 110000 \\
\hline \multicolumn{2}{|c|}{${ }^{39} \mathrm{Pu}$ Eqv $(\mathrm{Bg} / \mathrm{g})$ ? } & 2238298 & 53335,17 & 2858850 & 1535060 & 2347927 & 9110779 \\
\hline \multicolumn{2}{|c|}{ 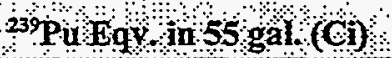 } & 8017 & 0041 & 0.22 & 0012 & 0.18 & 0074 \\
\hline
\end{tabular}

${ }^{a}$ Radionuclide-specific weighting factors for the ${ }^{239} \mathrm{Pu}$ equivalent activity taken from Appendix $\mathrm{A}$ of DOE/WIPP-069, Rev.5

There is concern about the thermal power from the decay heat of the radionuclides present in waste packages prepared for WIPP disposal. These concerns are addressed in Revision 5 of the WIPP WAC, with limits of 40 watts for a TRUPACT-II container for CH-TRU waste and a limit of 300 watts for a RH-TRU canister. High decay heat is also an indicator for potential problems with hydrogen gas generation. The major radionuclides found in the MVST sludge are listed in Table 21 along with the "Q" values needed to calculate the decay heat for each isotope.

An estimate of the decay heat distribution by radionuclide for the MVST sludge samples are listed in Table 22 along with an estimate for an upper boundary for total decay heat that would be in a 55 gal. drum full of wet sludge. These estimates indicate that the decay heat from MVST sludge is far below any of the WIPP WAC limits for thermal power and should have no impact on packaging requirements. For general interest, the relative percent distributions of the decay heat by radionuclide, beta activity, and alpha activity are listed in Table 23. The distribution of decay heat as a function of MVST tank and radionuclide is illustrated in Fig. 7 for beta decay, and in Fig. 8 for alpha decay. Although ${ }^{241} \mathrm{Pu}$ is a pure beta emitter, it is included with the other actinides for illustration. It is interesting to note that the beta activity dominates the decay heat output and that the heat from alpha decay is generally less than $10 \%$ of the total thermal power. 
Table 21 Isotopes that Contribute to the Decay Heat in the MVST Sludge

\begin{tabular}{|c|c|c|c|c|c|c|c|c|}
\hline Isotope & $\begin{array}{l}\text { "Q" value } \\
\text { (W/Ci) }\end{array}$ & $\begin{array}{l}\text { "Q" value } \\
(\mathrm{W} / \mathrm{Bq})\end{array}$ & $\begin{array}{c}W-24 \\
(\mathrm{Bg} / \mathrm{g})\end{array}$ & $\begin{array}{l}W-25 \\
(\mathrm{~Bq} / \mathrm{g})\end{array}$ & $\begin{array}{l}W-26 \\
(\mathrm{Bg} / \mathrm{g})\end{array}$ & $\begin{array}{l}W-27 \\
(\mathrm{Bg} / \mathrm{g})\end{array}$ & $\begin{array}{l}\text { W-28 } \\
\text { (Bq/g) }\end{array}$ & $\begin{array}{l}\text { W-31 } \\
(\mathrm{Bg} / \mathrm{g})\end{array}$ \\
\hline${ }^{60} \mathrm{Co}$ & $1.54 \mathrm{E}-02$ & $4.16 \mathrm{E}-13$ & $2.80 E+04$ & $2.50 E+04$ & $5.80 E+04$ & $1.20 E+04$ & $4.20 E+04$ & $2.20 E+04$ \\
\hline${ }^{90} \mathrm{Sr}$ & $1.16 E-03$ & $3.14 E-14$ & $1.40 E \div 06$ & $3.20 E+06$ & $7.10 E+05$ & $4.50 E+05$ & $7.00 E+05$ & $1.10 E+07$ \\
\hline${ }^{90} \mathrm{Y}$ & $5.54 \mathrm{E}-03$ & $1.50 \mathrm{E}-13$ & $1.40 \mathrm{E}+06$ & $3.20 E+06$ & $7.10 E+05$ & $4.50 E+05$ & $7.00 \mathrm{E}+05$ & $1.10 E+07$ \\
\hline${ }^{137} \mathrm{Cs}$ & $1.01 \mathrm{E}-03$ & 2.73E-14 & $5.30 \mathrm{E}+05$ & $4.70 E+05$ & $8.90 E+05$ & $3.90 \mathrm{E}+05$ & $3.10 E+05$ & $4.30 E+05$ \\
\hline${ }^{137 m} \mathrm{Ba}$ & $3.94 E-03$ & $1.06 E-13$ & $5.01 E+05$ & $4.45 E+05$ & $8.42 E+05$ & $3.69 E+05$ & $2.93 E+05$ & 4.07E+05 \\
\hline${ }^{152} \mathrm{Eu}$ & $7.65 E-03$ & 2.07E-13 & $8.90 \mathrm{E} \div 04$ & $7.10 E+04$ & $6.40 E+05$ & $4.10 E \div 04$ & $8.00 E+05$ & $3.00 E+04$ \\
\hline${ }^{15} \mathrm{Eu}$ & $9.08 \mathrm{E}-03$ & $2.45 E-13$ & $3.80 E+04$ & $3.70 \mathrm{E}+04$ & $2.90 E+04$ & $1.70 E+04$ & 2.70E+05 & $2.00 E+04$ \\
\hline${ }^{155} \mathrm{Eu}$ & 7.59E-04 & $2.05 E-14$ & $1.00 E+04$ & $8.40 \mathrm{E}+03$ & $6.30 E+04$ & $0.00 E+00$ & $7.00 \mathrm{E}+04$ & $0.00 E+00$ \\
\hline \multicolumn{3}{|c|}{ Total beta $(\mathrm{Ci} / \mathrm{Kg})$} & $1.08 E-01$ & 2.02E-01 & 1:07E-01 & $4.69 \mathrm{E}-02$ & $8: 64 E-02$ & $6: 20 \mathrm{E}-01$ \\
\hline 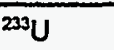 & $2.86 \mathrm{E}-02$ & $7.72 E-13$ & $1.60 E \div 03$ & $2.80 E+03$ & $1.00 E+04$ & $1.00 E \div 03$ & $5.20 \mathrm{E}+03$ & $5.20 \mathrm{E}+03$ \\
\hline${ }^{238} \mathrm{Pu}$ & 3.26E-02 & 8.81E-13 & $3.80 E+03$ & $7.80 \mathrm{E}+03$ & $5.40 E+03$ & $2.40 E+03$ & $3.00 \mathrm{E}+03$ & $1.30 E+04$ \\
\hline${ }^{239} \mathrm{Pu}$ & $3.02 E-02$ & 8.17E-13 & $1.90 E+03$ & $3.40 E+03$ & $1.30 \mathrm{E}+03$ & $6.70 \mathrm{E}+02$ & $8.30 E+02$ & $3.40 E+03$ \\
\hline${ }^{240} \mathrm{Pu}$ & $3.06 E-02$ & $8.26 \mathrm{E}-13$ & $8.70 \mathrm{E} \div 02$ & $1.80 \mathrm{E}+03$ & $8.90 E+02$ & $3.70 \mathrm{E}+02$ & $6.00 E+02$ & $2.20 E+03$ \\
\hline${ }^{241} \mathrm{Am}$ & $3.28 E-02$ & 8.87E-13 & $3.90 E+03$ & $9.30 E+03$ & $3.90 E+03$ & $2.80 E+03$ & $4.60 \mathrm{E}+03$ & $1.40 E+04$ \\
\hline${ }^{241} P u \beta^{-}$ & $3.20 \mathrm{E}-05$ & $8.65 E-16$ & $1.40 E+04$ & $2.60 E+04$ & $1.50 E+04$ & $6.50 \mathrm{E}+03$ & $1.20 E+04$ & $2.40 E+04$ \\
\hline${ }^{24} \mathrm{Cm}$ & 3.44E-02 & $9.29 \mathrm{E}-13$ & $2.20 \mathrm{E}+04$ & $5.80 E+04$ & $2.80 E+04$ & $1.70 \mathrm{E}+04$ & $2.50 \mathrm{E}+04$ & $1.10 \mathrm{E}+05$ \\
\hline \multicolumn{3}{|c|}{ Total arpha (Ci/Kg) } & 9.21E-0.4 & 2:25E-03 & 1:34E-03. & $6.55 E-04$ & $1 ; 06 E-03$ & $3.99 \mathrm{E}-03$ \\
\hline \multirow{2}{*}{\multicolumn{3}{|c|}{$\begin{array}{l}\text { Total beta in } 55 \text { gal. drum (Ci): } \\
\text { Total alpha in } 55 \text { gal. drum (Ci): }\end{array}$}} & 30.80 & 57.05 & 30.61 & 14.01 & 24.55 & 185.61 \\
\hline & & & 0.37 & 0.83 & 0.50 & 0.25 & 0.39 & 1.39 \\
\hline
\end{tabular}

Table 22 Distribution of Decay Heat in MVST Sludge

\begin{tabular}{|c|c|c|c|c|c|c|c|c|}
\hline Isotope & $\begin{array}{l}\text { "Q" value } \\
\text { (W/Ci) }\end{array}$ & $\begin{array}{c}\text { "Q" value } \\
(\mathrm{W} / \mathrm{Bq})\end{array}$ & $\begin{array}{l}W-24 \\
(W / K g)\end{array}$ & $\begin{array}{c}W-25 \\
(W / K g)\end{array}$ & $\begin{array}{c}W-26 \\
(W / K g)\end{array}$ & $\begin{array}{c}\text { W-27 } \\
(W / K g)\end{array}$ & $\begin{array}{c}W-28 \\
(W / K g)\end{array}$ & $\begin{array}{c}W-31 \\
(W / K g)\end{array}$ \\
\hline${ }^{60} \mathrm{Co}$ & $1.54 \mathrm{E}-02$ & $4.16 E-13$ & 1.17E-05 & $1.04 E-05$ & 2.42E-05 & $5.00 \mathrm{E}-06$ & 1.75E-05 & 9.16E-06 \\
\hline${ }^{90} \mathrm{Sr}$ & $1.16 E-03$ & 3.14E-14 & 4.39E-05 & $1.00 E-04$ & 2.23E-05 & 1.41E-05 & 2.19E-05 & $3.45 E-04$ \\
\hline soy & $5.54 E-03$ & $1.50 \mathrm{E}-13$ & $2.10 E-04$ & 4.79E-04 & $1.06 E-04$ & $6.74 E-05$ & $1.05 E-04$ & 1.65E-03 \\
\hline in $\mathrm{Cs}$ & 1.01E-03 & $2.73 E-14$ & $1.45 \mathrm{E}-05$ & $1.28 \mathrm{E}-05$ & $2.43 E-05$ & $1.06 E-05$ & $8.46 E-06$ & 1.17E-05 \\
\hline${ }^{137 \mathrm{~m}} \mathrm{Ba}$ & $3.94 E-03$ & $1.06 \mathrm{E}-13$ & $5.34 \mathrm{E}-05$ & 4.73E-05 & $8.97 E-05$ & $3.93 E-05$ & $3.12 E-05$ & 4.33E-05 \\
\hline 152 Eu & $7.65 \mathrm{E}-03$ & $2.07 E-13$ & $1.84 E-05$ & $1.47 E-05$ & $1.32 E-04$ & $8.47 E-06$ & $1.65 E-04$ & $6.20 \mathrm{E}-06$ \\
\hline${ }^{154} \mathrm{EU}$ & $9.08 E-03$ & $2.45 \mathrm{E}-13$ & $9.33 E-06$ & 9.08E-06 & 7.12E-06 & 4.17E-06 & $6.63 E-05$ & 4.91E-06 \\
\hline${ }^{155} \mathrm{Eu}$ & $7.59 E-04$ & $2.05 E-14$ & $2.05 E-07$ & $1.72 E-07$ & 1.29E-06 & $0.00 E+00$ & 1.44E-06 & $0.00 \mathrm{E}+00$ \\
\hline${ }^{23} \mathrm{U}$ & $2.86 \mathrm{E}-02$ & $7.72 E-13$ & $1.24 E-06$ & 2.16E-06 & $7.72 E-06$ & 7.72E-07 & $4.02 \mathrm{E}-06$ & 4.02E-06 \\
\hline${ }^{38} \mathrm{Pu}$ & 3.26E-02 & $8.81 E-13$ & $3.35 E-06$ & 6.87E-06 & $4.76 E-06$ & $2.11 E-06$ & $2.64 E-06$ & 1.15E-05 \\
\hline${ }^{209} \mathrm{Pu}$ & $3.02 E-02$ & $8.17 E-13$ & $1.55 E-06$ & $2.78 E-06$ & $1.06 E-06$ & $5.48 E-07$ & $6.78 E-07$ & $2.78 \mathrm{E}-06$ \\
\hline${ }^{210} \mathrm{Pu}$ & $3.06 E-02$ & $8.26 E-13$ & $7.19 E-07$ & 1.49E-06 & $7.35 E-07$ & $3.06 E-07$ & $4.96 E-07$ & $1.82 E-06$ \\
\hline${ }^{241} \mathrm{Am}$ & $3.28 E-02$ & 8.87E-13 & $3.46 E-06$ & 8.25E-06 & $3.46 E-06$ & $2.48 E-06$ & $4.08 E-06$ & $1.24 \mathrm{E}-05$ \\
\hline${ }^{241} P \cup \beta{ }^{\circ}$ & $3.20 \mathrm{E}-05$ & 8.65E-16 & $1.21 E-08$ & $2.25 E-08$ & $1.30 E-08$ & $5.62 E-09$ & $1.04 E-08$ & 2.08E-08 \\
\hline${ }^{24} \mathrm{Cm}$ & $3.44 \mathrm{E}-02$ & $9.29 \mathrm{E}-13$ & $2.04 E-05$ & 5.39E-05 & $2.60 E-05$ & $1.58 \mathrm{E}-05$ & $2.32 E-05$ & $1.02 E-04$ \\
\hline \multicolumn{2}{|l|}{ Total (W/Kg) } & $\because \div \quad$ & $3.92 E=04$ & $7.49 E=04$ & 4:51E-04 & 1.71E-04: & $4: 52 E-04$ & $220 \mathrm{E}-03$ \\
\hline Density & $(\mathrm{Kg} / \mathrm{L}):$ & & 1.37 & 1.36 & 1.38 & 1.44 & 1.37 & 1.44 \\
\hline \multicolumn{3}{|c|}{ Total in 55 gal drum $(\mathrm{Kg})$ : } & 285 & 283 & 287 & 300 & 285 & 300 \\
\hline \multicolumn{2}{|c|}{ Total in 55 gal drum (Watt): } & $\vdots \vdots$ & $.0: 112$ & 0.212 & 0.130 & 0.051 & $0: 129$ & 0.660 \\
\hline
\end{tabular}


Table 23 Summary of Relative Decay Heat in MVST Sludge

\begin{tabular}{|c|c|c|c|c|c|c|c|c|}
\hline Isotope & $\begin{array}{l}\text { "Q" value } \\
\text { (WICi) }\end{array}$ & $\begin{array}{c}\text { "Q" value } \\
(\mathrm{W} / \mathrm{Bq})\end{array}$ & $\begin{array}{c}\text { W-24 } \\
(\% \text { Watt })\end{array}$ & $\begin{array}{c}\text { W-25 } \\
(\% \text { Watt })\end{array}$ & $\begin{array}{c}\text { W-26 } \\
\text { (\% Watt) }\end{array}$ & $\begin{array}{c}\text { W-27 } \\
\text { (\% Watt) }\end{array}$ & $\begin{array}{c}\text { W-28 } \\
\text { (\% Watt) } \\
\end{array}$ & $\begin{array}{c}W-31 \\
(\% \text { Watt })\end{array}$ \\
\hline${ }^{60} \mathrm{Co}$ & $1.54 \mathrm{E}-02$ & $4.16 E-13$ & $2.98 \%$ & $1.39 \%$ & $5.35 \%$ & $2.92 \%$ & $3.87 \%$ & $0.42 \%$ \\
\hline${ }^{90} \mathrm{Sr}$ & $1.16 \mathrm{E}-03$ & $3.14 E-14$ & $11.20 \%$ & $13.39 \%$ & $4.93 \%$ & $8.25 \%$ & $4.85 \%$ & $15.66 \%$ \\
\hline soy & 5.54E-03 & $1.50 \mathrm{E}-13$ & $53.51 \%$ & $63.93 \%$ & $23.57 \%$ & $39.38 \%$ & $23.18 \%$ & $74.80 \%$ \\
\hline${ }^{137} \mathrm{Cs}$ & 1.01E-03 & 2.73E-14 & $3.69 \%$ & $1.71 \%$ & $5.39 \%$ & $6.22 \%$ & $1.87 \%$ & $0.53 \%$ \\
\hline $137 \mathrm{mBa}$ & $3.94 \mathrm{E}-03$ & $1.06 E-13$ & $13.63 \%$ & $6.32 \%$ & $19.87 \%$ & $22.96 \%$ & $6.91 \%$ & $1.97 \%$ \\
\hline 152Eu & 7.65E-03 & 2.07E-13 & $4.70 \%$ & $1.95 \%$ & $29.32 \%$ & $4.95 \%$ & $36.57 \%$ & $0.28 \%$ \\
\hline${ }^{154} \mathrm{Eu}$ & $9.08 E-03$ & 2.45E-13 & $2.38 \%$ & $1.21 \%$ & $1.58 \%$ & $2.44 \%$ & $14.66 \%$ & $0.22 \%$ \\
\hline${ }^{155} \mathrm{Eu}$ & 7.59E-04 & 2.05E-14 & $0.05 \%$ & $0.02 \%$ & $0.29 \%$ & $0.00 \%$ & $0.32 \%$ & $0.00 \%$ \\
\hline \multicolumn{2}{|c|}{ Total beta heat (\%): } & & $92.15 \%$ & $89.93 \%$ & $90.30 \%$ & $87.13 \%$ & $92.23 \%$ & $93.88 \%$ \\
\hline${ }^{2233} \mathrm{U}$ & 2.86E-02 & $7.72 E-13$ & $0.32 \%$ & $0.29 \%$ & $1.71 \%$ & $0.45 \%$ & $0.89 \%$ & $0.18 \%$ \\
\hline${ }^{238} \mathrm{Pu}$ & 3.26E-02 & 8.81E-13 & $0.85 \%$ & $0.92 \%$ & $1.05 \%$ & $1.24 \%$ & $0.58 \%$ & $0.52 \%$ \\
\hline${ }^{239} \mathrm{Pu}$ & $3.02 E-02$ & 8.17E-13 & $0.40 \%$ & $0.37 \%$ & $0.24 \%$ & $0.32 \%$ & $0.15 \%$ & $0.13 \%$ \\
\hline${ }^{240} \mathrm{Pu}$ & $3.06 E-02$ & 8.26E-13 & $0.18 \%$ & $0.20 \%$ & $0.16 \%$ & $0.18 \%$ & $0.11 \%$ & $0.08 \%$ \\
\hline${ }^{241} \mathrm{Am}$ & $3.28 \mathrm{E}-02$ & 8.87E-13 & $0.88 \%$ & $1.10 \%$ & $0.77 \%$ & $1.45 \%$ & $0.90 \%$ & $0.56 \%$ \\
\hline${ }^{241} \mathrm{Pu} \beta^{-}$ & $3.20 E-05$ & $8.65 E-16$ & $0.00 \%$ & $0.00 \%$ & $0.00 \%$ & $0.00 \%$ & $0.00 \%$ & $0.00 \%$ \\
\hline${ }^{2 \mu} \mathrm{Cm}$ & $3.44 \mathrm{E}-02$ & $9.29 E-13$ & $5.22 \%$ & $7.19 \%$ & $5.77 \%$ & $9.23 \%$ & $5.14 \%$ & $4.64 \%$ \\
\hline \multicolumn{3}{|c|}{ Total alpha heat (\%): } & $7.85 \%$ & $10.07 \%$ & $9.70 \%$ & $12.87 \%$ & $7.77 \%$ & $6.12 \%$ \\
\hline
\end{tabular}


Figure 7 Distribution of Beta Decay Heat in MVST Sludge

\section{MVST SLUDGE}

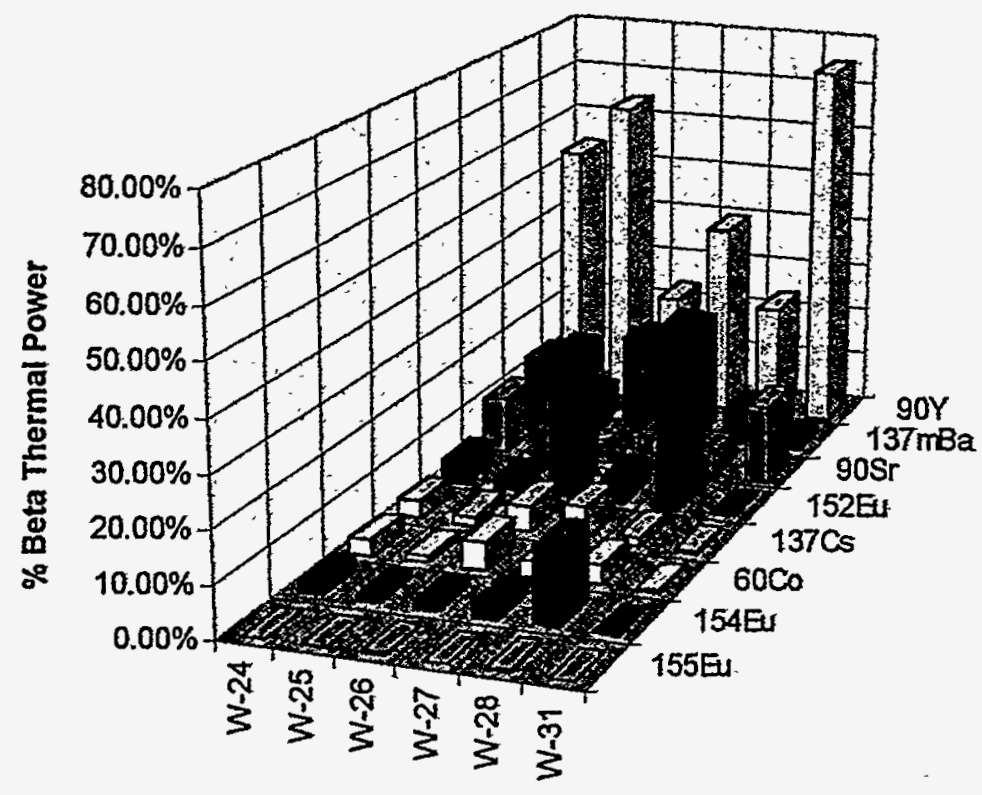

Figure 8 Distribution of Alpha Decay Heat in MVST Sludge

MVST SLUDGE

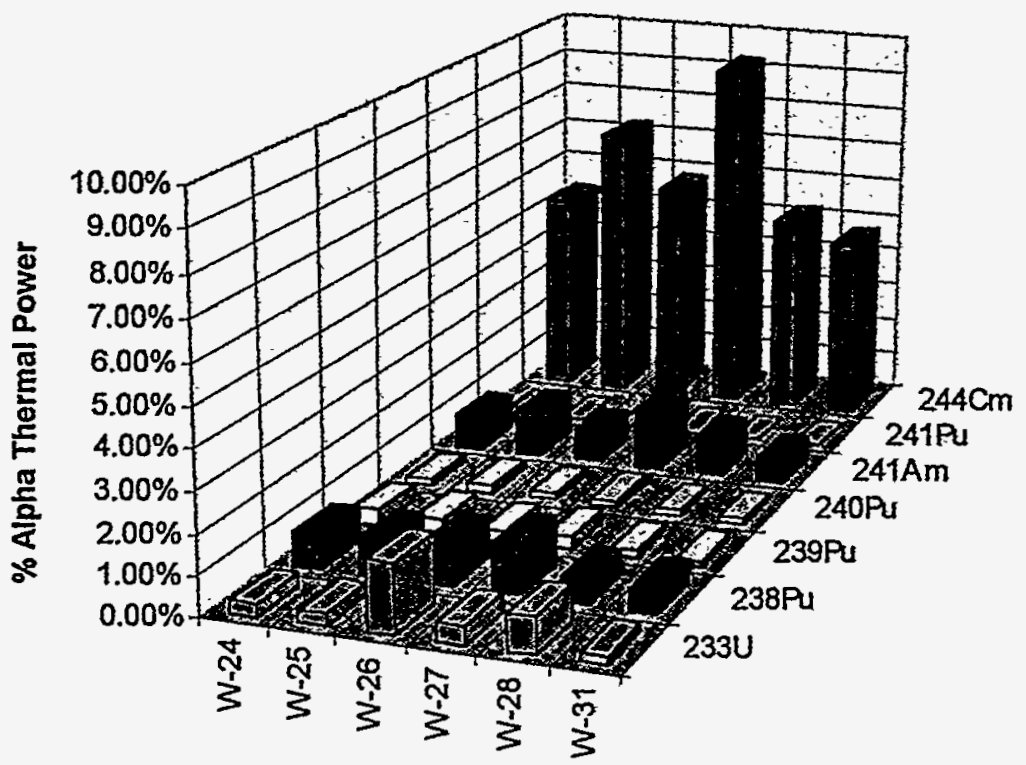




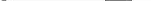




\subsection{Summary of Organic Analytical Results}

The organic content of the MVST samples was very low, with almost nothing above the detection limits observed in the supernatant and only trace amounts observed in the sludge samples. The few organic compounds observed consisted of products from the Purex and other actinide separation processes used by past chemical processing plants within the Laboratory. The target compound list (TCL) hits and the tentatively identified compounds (TIC) from the GC-MS analyses are listed in Table 24 for the supernatant samples and Table 25 for the sludge samples. For the organic chemical characterization results the following reporting conventions are used:

Reporting limits

B

D

$\mathbf{E}$

$\mathbf{J}$

$\mathbf{U}$

TIC
The reporting limits are the concentrations above which the response of the instrument for the calibrated range of concentrations is linear.

Data qualifier meaning that the compound was also found in the accompanying laboratory blank sample.

Data qualifier meaning sample dilution was required.

Data qualifier indicating that the reported concentration of the compound exceeded the calibration range of the instrument.

Data qualifier meaning that the compound was estimated at a concentration below the reporting limit; also used to indicate that the concentrations for tentatively identified compounds (TICs) are estimates.

Data qualifier meaning compound was not detected and method detection limits was reported.

Tentatively identified compound. The identification is based upon mass spectral data only, and the quantitation is based upon the response factor of the nearest eluting internal standard. All TIC values are estimates and are flagged with the " $\mathrm{J}$ " qualifier. 
4

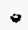

।

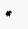

,

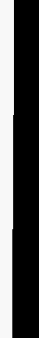


Table 24 Analytical Organic Data for MVST Liquid Samples

\begin{tabular}{|c|c|c|c|c|c|c|}
\hline \multirow{2}{*}{ Target Compound } & \multicolumn{6}{|c|}{ Concentration in Liquid, mg/L } \\
\hline & W-24 & W-25 & $W-26$ & W-27 & W-28 & W-31 \\
\hline \multicolumn{7}{|c|}{ Non-halogenated Volatile Organic Compounds $\left(\mathrm{N}\left(\mathrm{X}-\mathrm{V}_{\mathrm{OA}}\right)\right.$} \\
\hline Methanol & $2 \mathrm{U}$ & $2 \mathrm{U}$ & $2 \mathrm{U}$ & $2 U$ & $2 U$ & $2 U$ \\
\hline Acetone & $2 \mathrm{U}$ & $2 U$ & $2 U$ & $2 U$ & $2 \mathrm{U}$ & $2 U$ \\
\hline Methyl Ethyl Ketone & $2 U$ & $2 \mathrm{U}$ & $2 \mathrm{U}$ & $2 U$ & $2 U$ & $2 U$ \\
\hline Isobutanol & $2 \mathrm{U}$ & $2 \mathrm{U}$ & $2 \mathrm{U}$ & $2 U$ & $2 U$ & $2 \mathrm{U}$ \\
\hline Butanol & $2 \mathrm{U}$ & $2 U$ & $2 U$ & $2 U$ & $2 \mathrm{U}$ & $2 \mathrm{U}$ \\
\hline Pyridine & $2 \mathrm{U}$ & $2 \mathrm{U}$ & $2 U$ & $2 U$ & $2 \mathrm{U}$ & $2 U$ \\
\hline \multicolumn{7}{|c|}{ Yolatile Organic Compounds (VOA) } \\
\hline Vinyl Chloride & $1 \mathrm{U}$ & $1 \mathrm{U}$ & $1 \mathrm{U}$ & $1 U$ & $1 \mathrm{U}$ & $1 \mathrm{U}$ \\
\hline Trichlorofluoromethane & $1 \mathrm{U}$ & $1 \mathrm{U}$ & $1 \mathrm{U}$ & $1 \mathrm{U}$ & $1 \mathrm{U}$ & $1 \mathrm{U}$ \\
\hline Ethyl Ether & $1 U$ & $1 \mathrm{U}$ & $1 \mathrm{U}$ & $1 \mathrm{U}$ & $1 \mathrm{U}$ & $1 \mathrm{U}$ \\
\hline $\begin{array}{l}\text { 1,1,2-Trichloro-1,2,2- } \\
\text { trifluoroethane }\end{array}$ & $1 \mathrm{U}$ & $1 \mathrm{U}$ & $1 \mathrm{U}$ & $1 \mathrm{U}$ & $1 \mathrm{U}$ & $1 \mathrm{U}$ \\
\hline 1,2-Dichloroethylene & $1 \mathrm{U}$ & $1 \mathrm{U}$ & $1 \mathrm{U}$ & $1 \mathrm{U}$ & $1 U$ & $1 \dot{U}$ \\
\hline Methylene Chloride & $1 \mathrm{U}$ & $1 U$ & $1 \mathrm{U}$ & $1 \mathrm{U}$ & $1 \mathrm{U}$ & $1 \mathrm{U}$ \\
\hline Chloroform & $1 \mathrm{U}$ & $1 \mathrm{U}$ & $1 \mathrm{U}$ & $1 \mathrm{U}$ & $1 \mathrm{U}$ & $1 \mathrm{U}$ \\
\hline 1,2-Dichloroethane & $1 U$ & $1 \mathrm{U}$ & $1 \mathrm{U}$ & $1 \mathrm{U}$ & $1 \mathrm{U}$ & $1 \mathrm{U}$ \\
\hline 1,1,1-Trichloroethane & $1 \mathrm{U}$ & $1 \mathrm{U}$ & $1 \mathrm{U}$ & $1 \mathrm{U}$ & $1 U$ & $1 \mathrm{U}$ \\
\hline Carbon Tetrachloride & $1 \mathrm{U}$ & $1 \mathrm{U}$ & $1 \mathrm{U}$ & $1 \mathrm{U}$ & $1 \mathrm{U}$ & $1 U$ \\
\hline Benzene & $1 \mathrm{U}$ & $1 \mathrm{U}$ & $1 \mathrm{U}$ & $1 \mathrm{U}$ & $1 \mathrm{U}$ & $1 \mathrm{U}$ \\
\hline Trichloroethylene & $1 U$ & $1 U$ & $1 \mathrm{U}$ & $1 \mathrm{U}$ & $1 \mathrm{U}$ & $1 U$ \\
\hline 1,1,2-Trichloroethane & $1 \mathrm{U}$ & $1 U$ & $1 \mathrm{U}$ & $1 \mathrm{U}$ & $1 \mathrm{U}$ & $1 \mathrm{U}$ \\
\hline Bromoform & $1 \mathrm{U}$ & $1 \mathrm{U}$ & $1 \mathrm{U}$ & $1 \mathrm{U}$ & $1 \mathrm{U}$ & $1 \mathrm{U}$ \\
\hline Toluene & $1 \mathrm{U}$ & $1 \mathrm{U}$ & $1 \mathrm{U}$ & $1 \mathrm{U}$ & $1 U$ & $1 \mathrm{U}$ \\
\hline Tetrachloroethylene & $1 \mathrm{U}$ & $1 U$ & $1 \mathrm{U}$ & $1 \mathrm{U}$ & $1 \mathrm{U}$ & $1 \mathrm{U}$ \\
\hline Chlorobenzene & $1 \mathrm{U}$ & $1 U$ & $1 \mathrm{U}$ & $1 \mathrm{U}$ & $1 \mathrm{U}$ & $1 \mathrm{U}$ \\
\hline Ethylbenzene & $1 \mathrm{U}$ & $1 \mathrm{U}$ & $1 \mathrm{U}$ & $1 U$ & $1 \mathrm{U}$ & $1 \mathrm{U}$ \\
\hline m\&p-Xylenes & $1 \mathrm{U}$ & $1 \mathrm{U}$ & $1 \mathrm{U}$ & $1 \mathrm{U}$ & $1 \mathrm{U}$ & $1 U$ \\
\hline 0-Xylene & $1 \mathrm{U}$ & $1 \mathrm{U}$ & $1 \mathrm{U}$ & $1 \mathrm{U}$ & $1 \mathrm{U}$ & $1 \mathrm{U}$ \\
\hline 1,1,2,2-Tetrachloroethane & $1 \mathrm{U}$ & $1 \mathrm{U}$ & $1 \mathrm{U}$ & $1 U$ & $1 \mathrm{U}$ & $1 \mathrm{U}$ \\
\hline 1,4-Dichlorobenzene & $1 \mathrm{U}$ & $1 \mathrm{U}$ & $1 \mathrm{U}$ & $1 \mathrm{U}$ & $1 \mathrm{U}$ & $1 \mathrm{U}$ \\
\hline Ortho-Dichlorobenzene & $1 \mathrm{U}$ & $1 \mathrm{U}$ & $1 \mathrm{U}$ & $1 U$ & $1 \mathrm{U}$ & $1 \mathrm{U}$ \\
\hline
\end{tabular}




\begin{tabular}{|c|c|c|c|c|c|c|}
\hline \multirow{2}{*}{ Target Compound } & \multicolumn{6}{|c|}{ Concentration in Liquid, $\mathrm{mg} / \mathrm{L}$} \\
\hline & W-24 & W-25 & W-26 & W-27 & W-28 & W-31 \\
\hline \multicolumn{7}{|c|}{ Tentatively Ientiffed Yolatile Organic Compounds } \\
\hline Tetrahydrofuran & - & - & - & - & - & $0.01 \mathrm{~J}$ \\
\hline 3-Heptanone & - & - & - & - & - & $0.02 \mathrm{~J}$ \\
\hline Unknown & $0.04 \mathrm{~J}(3)^{2}$ & $0.06 \mathrm{~J}(3)^{\mathrm{a}}$ & $0.11 \mathrm{~J}(5)^{\mathrm{a}}$ & $0.24 \mathrm{~J}(9)^{\mathrm{a}}$ & $0.22 \mathrm{~J}(8)^{\mathrm{a}}$ & $0.3 \mathrm{~J}(11)^{\mathrm{a}}$ \\
\hline Unknown Hydrocarbon & - & - & - & - & $-\cdot$ & $0.03 \mathrm{~J}(2)^{\mathrm{a}}$ \\
\hline \multicolumn{7}{|c|}{ Sempolatile Organic Compounds(SVOA) } \\
\hline 2-Methyl Phenol & $0.05 \mathrm{U}$ & $0.05 \mathrm{U}$ & $0.05 \mathrm{U}$ & $0.05 \mathrm{U}$ & $0.05 \mathrm{U}$ & $0.05 \mathrm{U}$ \\
\hline Hexachloroethane & $0.05 \mathrm{U}$ & $0.05 \mathrm{U}$ & $0.05 \mathrm{U}$ & $0.05 \mathrm{U}$ & $0.05 \mathrm{U}$ & $0.05 U$ \\
\hline 4-Methyl Phenol & $0.05 \mathrm{U}$ & $0.05 \mathrm{U}$ & $0.05 \mathrm{U}$ & $0.05 \mathrm{U}$ & $0.05 \mathrm{U}$ & $0.05 \mathrm{U}$ \\
\hline Nitrobenzene & $0.05 \mathrm{U}$ & $0.05 \mathrm{U}$ & $0.05 \mathrm{U}$ & $0.05 \mathrm{U}$ & $0.05 \mathrm{U}$ & $0.05 \mathrm{U}$ \\
\hline 2,4-Dinitrotoluene & $0.05 \mathrm{U}$ & $0.05 \mathrm{U}$ & $0.05 \mathrm{U}$ & $0.05 \mathrm{U}$ & $0.05 \mathrm{U}$ & $0.05 \mathrm{U}$ \\
\hline 2,4-Dinitrophenol & $0.05 \mathrm{~J}$ & $0.05 \mathrm{U}$ & $0.05 \mathrm{U}$ & $0.30 \mathrm{~J}$ & $0.09 \mathrm{~J}$ & $0.41 \mathrm{~J}$ \\
\hline Hexachlorobenzene & $0.05 \mathrm{U}$ & $0.05 \mathrm{U}$ & $0.05 \mathrm{U}$ & $0.05 \mathrm{U}$ & $0.05 \mathrm{U}$ & $0.05 \mathrm{U}$ \\
\hline Pentachlorophenol & $0.05 \mathrm{U}$ & $0.05 \mathrm{U}$ & $0.05 \mathrm{U}$ & $0.05 \mathrm{U}$ & $0.05 \mathrm{~J}$ & $0.05 \mathrm{U}$ \\
\hline \multicolumn{7}{|c|}{ Tentatuyely rentiffed Semivolatile onganic Compounds o l } \\
\hline $\begin{array}{l}\text { Benzenesulfonamide, } \mathrm{N}- \\
\text { butyl }\end{array}$ & - & $0.47 \mathrm{~J}$ & - & - & $0.37 \mathrm{~J}$ & - \\
\hline Benzoic acid & - & - & - & - & - & $0.56 \mathrm{~J}$ \\
\hline 2-Butanamine & - & - & $0.03 \mathrm{~J}$ & - & - & - \\
\hline Dimethyl sulfone & - & - & $0.06 \mathrm{~J}$ & - & - & - \\
\hline Di-n-octyl phthalate & - & $0.40 \mathrm{~J}$ & $0.03 \mathrm{~J}$ & - & - & - \\
\hline Heptanal & $0.07 \mathrm{~J}$ & - & - & - & - & - \\
\hline Tributylphosphate (TBP) & $0.66 \mathrm{~J}$ & $1.1 \mathrm{~J}$ & - & $0.94 \mathrm{~J}$ & $0.14 \mathrm{~J}$ & $0.72 \mathrm{JD}$ \\
\hline Unknown & $2.0 \mathrm{~J}(19)^{2}$ & $4.6 \mathrm{~J}(16)^{\mathrm{a}}$ & $0.7 \mathrm{~J}(19)^{\mathrm{a}}$ & $4.9 \mathrm{~J}(19)^{2}$ & $5.8 \mathrm{~J}(19)^{\mathrm{a}}$ & $10 \mathrm{~J}(20)^{\mathrm{a}}$ \\
\hline
\end{tabular}

a Number of compounds grouped together listed in parenthesis. 
Table 25 Analytical Organic Data for MVST Sludge Samples

\begin{tabular}{|c|c|c|c|c|c|c|}
\hline \multirow{2}{*}{ Target Compound } & \multicolumn{6}{|c|}{ Concentration in Sludge, $\mathrm{mg} / \mathrm{Kg}$} \\
\hline & W-24 & W-25 & W-26 & W-27 & W-28 & W-31 \\
\hline \multicolumn{7}{|c|}{ Semivolätile Organic Compounds (SVOA) } \\
\hline 2-Methyl Phenol & $5 \mathrm{U}$ & $10 \mathrm{UD}$ & $5 U$ & $5 \mathrm{U}$ & $5 U$ & $5 \mathrm{U}$ \\
\hline Hexachloroethane & $5 U$ & $10 \mathrm{UD}$ & $5 \mathrm{U}$ & $5 \mathrm{U}$ & $5 \mathrm{U}$ & $5 \mathrm{U}$ \\
\hline 4-Methyl Phenol & $5 \mathrm{U}$ & $10 \mathrm{UD}$ & $5 U$ & $5 U$ & $5 \mathrm{U}$ & $5 \mathrm{U}$ \\
\hline Nitrobenzene & $5 U$ & $10 \mathrm{UD}$ & $5 \mathrm{U}$ & $5 U$ & $5 U$ & $5 \mathrm{U}$ \\
\hline 2,4-Dinitrotoluene & $0.3 \mathrm{U}$ & $0.6 \mathrm{UD}$ & $0.3 \mathrm{U}$ & $0.3 \mathrm{U}$ & $0.3 \mathrm{U}$ & $0.3 \mathrm{U}$ \\
\hline 2,4-Dinitrophenol & $5 \mathrm{U}$ & $10 \mathrm{UD}$ & $5 \mathrm{U}$ & $5 \mathrm{U}$ & $5 \mathrm{U}$ & $5 \mathrm{U}$ \\
\hline Hexachlorobenzene & $0.3 \mathrm{U}$ & $0.6 \mathrm{UD}$ & $0.3 \mathrm{U}$ & $0.3 \mathrm{U}$ & $0.3 \mathrm{U}$ & $0.3 \mathrm{U}$ \\
\hline Pentachlorophenol & $5 \mathrm{U}$ & $10 \mathrm{UD}$ & $5 \mathrm{U}$ & $5 U$ & $5 \mathrm{U}$ & $5 \mathrm{U}$ \\
\hline \multicolumn{7}{|c|}{ Tentatively Identified Semivolatile Organic Compounds } \\
\hline Benzene, diethyl- & $7.4 \mathrm{~J}(2)^{\mathrm{a}}$ & $21 \mathrm{JD}(2)^{\mathrm{a}}$ & - & - & - & - \\
\hline $\begin{array}{l}\text { Benzene, 1,3-bis(1- } \\
\text { methylethyl)- }\end{array}$ & $3.0 \mathrm{~J}$ & $9.0 \mathrm{JD}$ & - & - & - & - \\
\hline Benzophenone & - & - & $0.99 \mathrm{~J}$ & $1.0 \mathrm{~J}$ & $1.1 \mathrm{~J}$ & $2.8 \mathrm{~J}$ \\
\hline Dibutyl phthalate & - & - & $1.4 \mathrm{~J}$ & $1.2 \mathrm{~J}$ & $1.2 \mathrm{~J}$ & - \\
\hline 1-Docosene & - & - & $1.2 \mathrm{~J}$ & - & - & - \\
\hline Dodecane & $3.5 \mathrm{~J}$ & $7.3 \mathrm{JD}$ & $1.0 \mathrm{~J}$ & $1.9 \mathrm{~J}$ & $0.7 \mathrm{~J}$ & $3.5 \mathrm{~J}$ \\
\hline 1-Dotriacontanol & - & - & - & $1.2 \mathrm{~J}$ & - & - \\
\hline $\begin{array}{l}\text { Ethanone, } 1-(4- \\
\text { ethylphenyl)- }\end{array}$ & - & - & $1.3 \mathrm{~J}$ & - & - & - \\
\hline Heptadecane & - & - & - & $0.9 \mathrm{~J}$ & - & - \\
\hline $\begin{array}{l}\text { Heptane, 4-ethyl-2,2,6,6- } \\
\text { tetramethyl }\end{array}$ & $3.6 \mathrm{~J}$ & - & - & - & - & $5.3 \mathrm{~J}$ \\
\hline 1-Hexanol, 2-ethyl & - & - & - & - & $0.54 \mathrm{~J}$ & - \\
\hline Hexadecanoic acid & - & - & $1.8 \mathrm{~J}$ & - & - & - \\
\hline Nonadecane & - & - & - & $1.1 \mathrm{~J}$ & - & - \\
\hline I- Nonadecanol & - & - & $3.1 \mathrm{~J}$ & - & - & - \\
\hline Octadecane & - & - & - & $1.6 \mathrm{~J}$ & - & - \\
\hline $\begin{array}{l}\text { 1-Octanamine, N-nitroso- } \\
\text { n-octyl- }\end{array}$ & $1.4 \mathrm{~J}$ & - & - & - & - & - \\
\hline Pentadecane & - & - & - & $1.1 \mathrm{~J}$ & - & - \\
\hline Tetradecane & $8.7 \mathrm{~J}$ & $11 \mathrm{JD}$ & $2.3 \mathrm{~J}$ & $3.7 \mathrm{~J}$ & $1.5 \mathrm{~J}$ & $2.3 \mathrm{~J}$ \\
\hline
\end{tabular}




\begin{tabular}{|c|c|c|c|c|c|c|}
\hline \multirow{2}{*}{ Target Compound } & \multicolumn{6}{|c|}{ Concentration in Sludge, $\mathrm{mg} / \mathrm{Kg}$} \\
\hline & W-24 & W-25 & W-26 & $\mathbf{W}-27$ & W-28 & W-31 \\
\hline Tributylphosphate (TBP) & $2.5 \mathrm{~J}$ & $14 \mathrm{JD}$ & - & - & $2.1 \mathrm{~J}$ & $15 \mathrm{~J}$ \\
\hline Tridecane & $11 \mathrm{~J}$ & $13 \mathrm{JD}$ & $2.2 \mathrm{~J}$ & $4.5 \mathrm{~J}$ & $1.4 \mathrm{~J}$ & $3.6 \mathrm{~J}$ \\
\hline Undecane & $3.3 \mathrm{~J}$ & $6.8 \mathrm{~J}$ & - & $1.7 \mathrm{~J}$ & - & $5.1 \mathrm{~J}$ \\
\hline Unknown Hydrocarbons & $4 \mathrm{~J}(2)^{\mathrm{a}}$ & $56 \mathrm{JD}(9)^{2}$ & - & - & - & $28 \mathrm{~J}(8)^{2}$ \\
\hline \multicolumn{7}{|c|}{ Polychlorinated Bhphenyls (PCB) Analysis , } \\
\hline Aroclor-1016 & $0.048 \mathrm{U}$ & $0.050 \mathrm{U}$ & $\mathrm{nd}^{\mathrm{b}}$ & nd & $0.050 \mathrm{U}$ & $0.050 \mathrm{U}$ \\
\hline Aroclor-1221 & $0.048 \mathrm{U}$ & $0.050 \mathrm{U}$ & nd & nd & $0.050 \mathrm{U}$ & $0.050 \mathrm{U}$ \\
\hline Aroclor-1232 & $0.048 \mathrm{U}$ & $0.050 \mathrm{U}$ & nd & nd & $0.050 \mathrm{U}$ & $0.050 \mathrm{U}$ \\
\hline Aroclor- 1242 & $0.049 \mathrm{~J}$ & $0.050 \mathrm{U}$ & $0.050 \mathrm{U}$ & $0.050 \mathrm{U}$ & $0.050 \mathrm{U}$ & $0.050 \mathrm{U}$ \\
\hline Aroclor- 1248 & $0.048 \mathrm{U}$ & $0.050 \mathrm{U}$ & nd & nd & $0.050 \mathrm{U}$ & $0.050 \mathrm{U}$ \\
\hline Aroclor-1254 & $0.048 \mathrm{U}$ & $0.050 \mathrm{U}$ & $0.050 \mathrm{U}$ & $0.050 \mathrm{U}$ & $0.050 \mathrm{U}$ & $0.050 \mathrm{U}$ \\
\hline Aroclor- 1260 & $0.048 \mathrm{U}$ & $0.050 \mathrm{U}$ & nd & nd & $0.050 \mathrm{U}$ & $0.050 \mathrm{U}$ \\
\hline
\end{tabular}

${ }^{a}$ Number of compounds grouped together listed in parenthesis.

${ }^{\mathrm{b}}$ Compounds not detected and calibration was not available to calculate detection limit. 


\subsection{Discussion of Organic Analysis}

Some difficulties were encountered during the extraction preparation for samples W-26 and W-28 supernatant samples. These problems appear to have impacted the semivolatile and PCB analysis for W-26 as explained below. Although the hold time requirements from the sample collection to the sample extraction were satisfied, the hold time from extraction to analysis for PCB analysis was exceeded by thirteen days for sludge samples from tanks W-28 and W-31 because of instrument downtime. The surrogate recoveries for these samples were within control limits and thus there is considered to be no impact to data quality due to the missed holding time.

During the extraction of the W-26 liquid sample the extraction mixture formed three layers. The bottom and middle layers appeared to be emulsions and the top layer was clear. The top layer was determined to be methylene chloride, which was not expected because methylene chloride is more dense than water. Additional methylene chloride extractions were performed on the emulsions to try to recovery any organic compounds which may have been trapped in the emulsified layers. Including all attempts to recover the methylene chloride, only $50 \%$ of the total volume used in the extraction was actually recovered. The PCB surrogate recoveries were below the control limits for this sample. The semivolatile surrogate recoveries were comparable to the other MVST supernatant samples but still were low.

During the W-28 extraction there was no layer formation after the addition of methylene chloride even after centrifuging the extraction. It is interesting to note that the sample density was determined to be $1.34 \mathrm{~g} / \mathrm{mL}$ for the W-28 liquid sample which is the same density of methylene chloride at 20 ${ }^{\circ} \mathrm{C}$. This similarity in density explains the absence of any separation of the organic and aqueous phase. The sample was acidified using sulfuric acid which increased the density of the aqueous phase and an organic phase separated out on top of the aqueous. After acidification the added methylene chloride volume was fully recovered and the subsequent extractions were successfully performed on the acidified sample portion. The PCB and semivolatile surrogate recoveries for this sample were comparable to other MVST supernatant samples. 


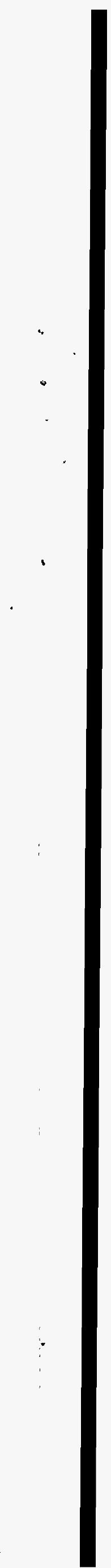




\section{REFERENCES}

1. T. D. Hylton, Sampling and Analysis Plan for the Bethel Valley Evaporator Service Tanks and the Melton Valley Storage Tanks, ORNL/M-5224, July 1996.

2. F. J. Peretz, B. R. Clark, C. B. Scott, and J. B. Berry, Characterization of Low-Level Liquid Wastes at the Oak Ridge National Laboratory, ORNL/TM-10218, December 1986.

3. M. B. Sears, J. L. Botts, R. N. Ceo, J.J. Ferrada, W. H. Griest, J. M. Keller, and R. L. Schenley, Sampling and Analysis of Radioactive Liquid Wastes and Sludges in the Melton Valley and Evaporator Facility Storage Tanks at ORNL, ORNL/TM-11652, September 1990.

4. J. M. Keller, J. M. Giaquinto, and W. H. Griest, Characterization of Selected Waste Tanks from the Active LLLW System, ORNL/TM-13248, August 1996.

5. M. B. Sears, Results of Sampling the Contents of the Liquid Low-Level Waste Evaporator Feed Tank W-22, ORNL/TM-13234, September 1996.

6. S. M. DePaoli, Oak Ridge National Laboratory, personal communication to J. M Keller, December 12, 1996.

7. U. S. Environmental Protection Agency, Test Methods for Evaluating Solid Waste, SW-846, 3rd ed, Office of Solid Waste and Emergency Response, Washington, D.C., November 1986; Update I, July 1992; and Final Update II, September 1994.

8. J. M. Giaquinto, A. M. Essling, and J. M. Keller, Comparison of SW-846 Method 3051 and SW-846 Method 7471A for the Preparation of Solid Waste Samples for Mercury Determination, ORNL/TM-13236, July 1996.

9. Transuranic Waste Characterization Quality Assurance Program Plan, Rev. 0, CAO-941010, April 30, 1995

10. Radioactive Materials Analysis Laboratory - Oak Ridge National Laboratory (RMALORNL) Quality Assurance Project Plan (QAPjP) for the Transuranic Waste Characterization Program (TWCP), Rev. 1, QAP-X-CASD/RML-002, January 1996.

11. Radioactive Materials Analysis Laboratory Quality Assurance Plan for the Characterization of Radioactive Waste, QAP-X-CASD/RML-001, Rev. 1, June 1996

12. Waste Acceptance Criteria for the Waste Isolation Pilot Plant, WIPP-DOE-069, Rev. 5.0, April 1996. 


\section{APPENDIX A}

\section{Radioactive Materials Analytical Laboratory QC Acceptance Criteria for Radioactive Liquid/Solid Waste Samples}

\begin{tabular}{|c|c|c|c|c|}
\hline Analysis & $\begin{array}{l}\text { Method (s) } \\
\text { CASD-AM- }\end{array}$ & $\begin{array}{l}\text { Quality Control } \\
\text { Check } \\
\text { (per batch) }\end{array}$ & $\begin{array}{c}\text { SW-846 } \\
\text { Acceptance } \\
\text { Criteria } \\
(\% D, \% R, R P D)^{e}\end{array}$ & $\begin{array}{c}\text { RMAL } \\
\text { Acceptance } \\
\text { Criteria } \\
(\% \mathbf{D}, \% \mathbf{R}, \mathbf{R P D})^{\mathbf{e}}\end{array}$ \\
\hline $\begin{array}{l}\text { Metals by ICP-AES } \\
\text { (inductively } \\
\text { coupled plasma } \\
\text { atomic emission } \\
\text { spectroscopy) }\end{array}$ & SW846-6010A & $\begin{array}{l}\text { high standard } \\
\text { calibration verifications (ICV \& CCV) } \\
\text { calibration blank \& checks (ICB \& CCB) } \\
\text { method blank (sample prep) } \\
\text { matrix spike } \\
\text { matrix spike duplicate or sample duplicate } \\
\text { laboratory control sample (sample prep) } \\
\text { serial dilution (if interference suspected) } \\
\text { post digestion spiket }\end{array}$ & $\begin{array}{l} \pm 5 \% \mathrm{D} \\
\pm 10 \% \mathrm{D} \\
\leqslant \times \mathrm{IDL} \\
\leqslant \times \mathrm{IDL} \\
\pm 20 \% \mathrm{D} \\
\pm 20 \mathrm{RPD} \\
\text { none specified } \\
\pm 10 \% \mathrm{R} \\
\pm 20 \% \mathrm{D}\end{array}$ & $\begin{array}{c} \pm 5 \% \mathrm{D} \\
\pm 10 \% \mathrm{D} \\
\leqslant \times \mathrm{IDL} \\
3 \times \mathrm{IDL} \\
\pm 25 \% \mathrm{D} \text { (liq.), } \neq 30 \% \mathrm{D} \text { (solid) } \\
\pm 20 \mathrm{RPD} \text { (liq.), } \pm 30 \mathrm{RPD} \text { (solid) } \\
\pm 20 \% \mathrm{D} \\
\pm 10 \% \mathrm{R} \\
\pm 25 \% \mathrm{D} \text { (liq.) })_{2} \neq 30 \% \mathrm{D} \text { (solid) }\end{array}$ \\
\hline $\begin{array}{l}\text { Metals by ICP-MS } \\
\text { (inductively } \\
\text { coupled plasma- } \\
\text { mass spectrometry) }\end{array}$ & SW846-6020 & $\begin{array}{l}\text { calibration verifications (ICV \& CCV) } \\
\text { calibration blank \& blank checks (CCB)" } \\
\text { method blank (sample prep) } \\
\text { matrix spike } \\
\text { matrix spike duplicate or sample duplicate } \\
\text { laboratory control sample (sample prep) } \\
\text { internal standard } \\
\text { post digestion spike }\end{array}$ & $\begin{array}{l} \pm 10 \% \mathrm{D} \\
8 \times \mathrm{IDL} \\
\text { none specified } \\
\text { none specified } \\
\pm 20 \mathrm{RPD} \\
\text { none specified } \\
30-120 \% \mathrm{R} \\
\pm 10 \% \mathrm{D}\end{array}$ & $\begin{array}{c} \pm 10 \% \mathrm{D} \\
<\times \mathrm{IDL} \\
<10 \times \mathrm{IDL} \\
\pm 25 \% \mathrm{D} \text { (liq.) } \pm 30 \% \mathrm{DD} \text { (solid) } \\
\pm 20 \mathrm{RPD} \text { (liq.), } \pm 30 \mathrm{RPD} \text { (solid) } \\
\pm 20 \% \mathrm{D} \\
\pm 30 \% \mathrm{D} \\
\pm 20 \% \mathrm{D}\end{array}$ \\
\hline $\begin{array}{l}\text { Metals by GFAA } \\
\text { (graphite furmace } \\
\text { atomic absorption) }\end{array}$ & SW846-7000A & $\begin{array}{l}\text { high standard } \\
\text { calibration verifications (ICV \& CCV)' } \\
\text { method blank (sample prep) } \\
\text { matrix spike } \\
\text { matrix spike duplicate } \\
\text { laboratory control sample (sample prep) } \\
\text { serial dilution (if interference suspected) } \\
\text { post digestion spike }\end{array}$ & $\begin{array}{l}\text { not required } \\
=10 \% \mathrm{D} \text { (ICV), }=20 \% \mathrm{D}(\mathrm{CCV}) \\
\text { none specified } \\
\text { none specified } \\
\text { none specified } \\
\text { none specified } \\
\pm 10 \% \mathrm{R} \\
\pm 15 \% \mathrm{D}\end{array}$ & 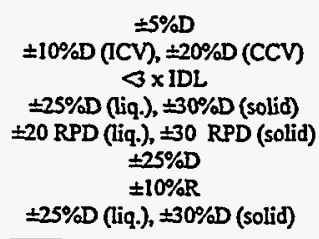 \\
\hline $\begin{array}{l}\text { Mereury by CVAA } \\
\text { (cold vapor atomic } \\
\text { absorption) }\end{array}$ & $\begin{array}{l}\text { SW846-7471A } \\
\text { SW846-7470 }\end{array}$ & $\begin{array}{l}\text { instrument blank } \\
\text { calibration verification (ICV \& CCV) } \\
\text { method blank (sample prep) } \\
\text { laboratory control sample (sample prep)c } \\
\text { matrix spike } \\
\text { matrix spike duplicate or sample duplicate } \\
\text { post digestion spike‘ }\end{array}$ & $\begin{array}{l}\text { none specified } \\
\text { none specified } \\
\text { none specified } \\
\text { none specified } \\
\text { none specified } \\
\text { none specified } \\
\text { none specified }\end{array}$ & 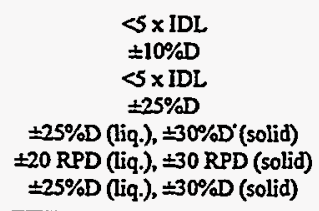 \\
\hline $\begin{array}{l}\text { Carbon (total } \\
\text { organic carbon, } \\
\text { total catoon, total } \\
\text { inorganic carbon) }\end{array}$ & SW846-9060 & $\begin{array}{l}\text { instrument blank. } \\
\text { calibration verification (ICV \& CCV) } \\
\text { matrix spike } \\
\text { matrix spike duplicate }\end{array}$ & $\begin{array}{l}\text { none specified } \\
\text { none specified } \\
\text { none specified } \\
\text { none specified }\end{array}$ & $\begin{array}{c}\beta \times 1 \mathrm{DL} \\
=10 \% \mathrm{DD} \text { (ICV.), } \pm 20 \% \mathrm{DD} \text { (CCV) } \\
\pm 25 \% \mathrm{D} \text { (liq.), } \pm 30 \% \mathrm{D} \text { (solid) } \\
\pm 20 \mathrm{RPD} \text { (liq.), } \neq 30 \mathrm{RPD} \text { (solid) }\end{array}$ \\
\hline $\begin{array}{l}\text { Anions by lon } \\
\text { Chromatography } \\
\text { (IC) }\end{array}$ & SW846-9056 & $\begin{array}{l}\text { calibration verification (ICV \& CCV) } \\
\text { matrix spike } \\
\text { sample duplicate }\end{array}$ & $\begin{array}{c} \pm 10 \% \mathrm{D} \text { (ICV), } \pm 5 \% \mathrm{D}(\mathrm{CCV}) \\
\text { none specified } \\
\text { none specified }\end{array}$ & $\begin{array}{c} \pm 10 \% \mathrm{D}(\mathrm{ICV}), \pm 15 \% \mathrm{D}(\mathrm{CCV}) \\
\pm 25 \% \mathrm{D} \\
\pm 20 \mathrm{RPD}\end{array}$ \\
\hline $\mathrm{pH}$ measurement & $\begin{array}{l}\text { SW846-9040A } \\
\text { SW846-9045B }\end{array}$ & $\begin{array}{l}\text { check standard } \\
\text { sample duplicate }\end{array}$ & $\begin{array}{l}\text { none specified } \\
\text { none specified }\end{array}$ & $\begin{array}{l} \pm 10 \% \mathrm{D} \\
\pm 20 \% \mathrm{D}\end{array}$ \\
\hline $\begin{array}{l}\text { Total and dissolved } \\
\text { solids (TS \& TDS) }\end{array}$ & $\begin{array}{l}\text { EPA600-160.2 } \\
\text { EPA600-160.3 }\end{array}$ & $\begin{array}{l}\text { sample duplicate } \\
\text { check standard }\end{array}$ & $\begin{array}{l}\text { none specified } \\
\text { none specified }\end{array}$ & $\begin{array}{c}=10 \mathrm{mg} / 10 \mathrm{~mL} \text { sample } \\
\quad \neq 10 \% \mathrm{D}\end{array}$ \\
\hline $\begin{array}{l}\text { Carbonate and } \\
\text { bicarbonate titration }\end{array}$ & AC-MM-1 003105 & $\begin{array}{l}\text { sample duplicate } \\
\text { check standard }\end{array}$ & $\begin{array}{l}\text { none specified } \\
\text { none specified }\end{array}$ & $\begin{array}{l} \pm 20 \mathrm{RPD} \\
\pm 20 \% \mathrm{D}\end{array}$ \\
\hline Gross alpha/beta & $\begin{array}{l}\text { EPA-900.0 } \\
\text { RML-RA02 } \\
\text { RML-RA12 }\end{array}$ & $\begin{array}{l}\text { background check } \\
\text { calibration verification } \\
\text { method blank (optional)' } \\
\text { sample duplicate } \\
\text { matrix spike }\end{array}$ & $\begin{array}{l}\text { none specified } \\
\text { none specified } \\
\text { none specified } \\
\text { none specified } \\
\text { none specified }\end{array}$ & $\begin{array}{c}\text { <3sigma daily change } \\
\pm 10 \% \mathrm{D} \\
\text { evaluated for contamination } \\
\pm 25 \mathrm{RPD} \text { (liq.), } \pm 30 \mathrm{RPD} \text { (solid) } \\
\pm 25 \% \mathrm{D} \text { (liq.) } \& \pm 30 \% \mathrm{D} \text { (solid) }\end{array}$ \\
\hline $\begin{array}{l}\text { Nuclides by gamma } \\
\text { spectrometry }\end{array}$ & EPA-901.1 & $\begin{array}{l}\text { background check } \\
\text { calibration verification } \\
\text { sample duplicate }\end{array}$ & $\begin{array}{l}\text { none specified } \\
\text { none specified } \\
\text { none specified }\end{array}$ & $\begin{array}{c}\text { <3sigma daily change } \\
\quad=10 \% \mathrm{D} \\
\pm 25 \% \mathrm{D} \text { (iiq.) } \& \pm 30 \% \mathrm{D} \text { (solid) }\end{array}$ \\
\hline
\end{tabular}




\begin{tabular}{|c|c|c|c|c|}
\hline Analysis & $\begin{array}{l}\text { Method (s) } \\
\text { CASD-AM- }\end{array}$ & $\begin{array}{l}\text { Quality Control } \\
\text { Check } \\
\text { (per batch) }\end{array}$ & $\begin{array}{c}\text { SW-846 } \\
\text { Acceptance } \\
\text { Criteria } \\
(\% D, \% \text { RPD })^{e}\end{array}$ & $\begin{array}{c}\text { RMAL } \\
\text { Acceptance } \\
\text { Criteria } \\
(\% \mathbf{O}, \% \mathbf{R}, \mathbf{R P D})^{\mathbf{c}}\end{array}$ \\
\hline Sr-90 determination & $\begin{array}{l}\text { RML-RA13 } \\
\text { EPA-905.0 }\end{array}$ & $\begin{array}{l}\text { method blank (optional) } \\
\text { laboratory control sample } \\
\text { matrix spike } \\
\text { matrix spike duplicate or sample duplieate } \\
\text { associated instrument QC }\end{array}$ & $\begin{array}{l}\text { none specified } \\
\text { none specified } \\
\text { none specified } \\
\text { none specified } \\
\text { none specified }\end{array}$ & 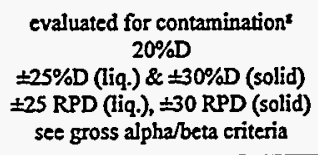 \\
\hline $\begin{array}{l}\text { Tc-99 } \\
\text { determination }\end{array}$ & $\begin{array}{l}\text { DOE Compendium } \\
\text { RPS50 } \\
\text { RML-RA05 }\end{array}$ & $\begin{array}{l}\text { method blank (optional) } \\
\text { laboratory control sample } \\
\text { matrix spike } \\
\text { matrix spike or sample duplicate } \\
\text { associated instrument QC }\end{array}$ & $\begin{array}{l}\text { none specified } \\
\text { none specified } \\
\text { none specified } \\
\text { none specified } \\
\text { none specified }\end{array}$ & $\begin{array}{c}<3 \times \text { IDL } \\
20 \% \mathrm{D} \\
\pm 25 \% \mathrm{D} \text { (liq.) \& } \pm 30 \% \mathrm{D} \text { (solid) } \\
\pm 25 \mathrm{RPD} \text { (lig.), } \pm 30 \mathrm{RPD} \text { (solid) } \\
\text { sec ICP-MS criteria }\end{array}$ \\
\hline H-3 determination & EPA-906.0 & $\begin{array}{l}\text { method blank (optional) } \\
\text { laboratory control sample } \\
\text { matrix spike } \\
\text { matrix spike duplicate or sample duplicate } \\
\text { associated instrument } Q C\end{array}$ & $\begin{array}{l}\text { none specified } \\
\text { none specified } \\
\text { none specified } \\
\text { none specified } \\
\text { none specified }\end{array}$ & $\begin{array}{l}\text { evaluated for contamination } \\
\qquad 20 \% \mathrm{D} \\
\pm 25 \% \mathrm{D} \text { (liq.) \& } \pm 30 \% \mathrm{D} \text { (solid) } \\
\pm 25 \text { RPD (liq.), } \pm 30 \text { RPD (solid) } \\
\text { see gross alpha/beta criteria }\end{array}$ \\
\hline $\mathrm{Cm}-244$ & RML-RA06 & $\begin{array}{l}\text { method blank (optional) } \\
\text { laboratory control sample } \\
\text { matrix spike } \\
\text { matrix spike duplicate or sample duplicate } \\
\text { associated instrument } Q C\end{array}$ & $\begin{array}{l}\text { none specified } \\
\text { none specified } \\
\text { none specified } \\
\text { none specified } \\
\text { none specified }\end{array}$ & $\begin{array}{l}\text { evaluated for contamination } \\
\qquad 20 \% \mathrm{D} \\
\pm 25 \% \mathrm{D} \text { (liq.) \& } \pm 30 \% \mathrm{D} \text { (solid) } \\
\pm 25 \text { RPD (liq.), } \pm 30 \text { RPD (solid) } \\
\text { see gross alpha/beta criteria }\end{array}$ \\
\hline $\mathrm{Pu}-238,239 / 240$ & $\begin{array}{l}\text { RML-RA11 } \\
\text { RML-RA08 }\end{array}$ & $\begin{array}{l}\text { method blank (optional) } \\
\text { laboratory control sample } \\
\text { matrix spike } \\
\text { matrix spike duplicate or sample duplicate } \\
\text { associated instrument QC }\end{array}$ & $\begin{array}{l}\text { none specified } \\
\text { none specified } \\
\text { none specified } \\
\text { none specified } \\
\text { none specified }\end{array}$ & $\begin{array}{l}\text { evaluated for contaminations } \\
\qquad 20 \% \mathrm{D} \\
\pm 25 \% \mathrm{D} \text { (liq.) \& } \pm 30 \% \mathrm{D} \text { (solid) } \\
\pm 25 \text { RPD (liq.), } \pm 30 \mathrm{RPD} \text { (solid) } \\
\text { see gross alpha/beta criteria }\end{array}$ \\
\hline$U-233 / 234$ & RML-RA10 & $\begin{array}{l}\text { method blank (optiona) } \\
\text { laboratory control sample } \\
\text { matrix spike } \\
\text { matrix spike duplicate or sample duplicate } \\
\text { associated instrument } Q C\end{array}$ & $\begin{array}{l}\text { none specified } \\
\text { none specified } \\
\text { none specified } \\
\text { none specified } \\
\text { none specified }\end{array}$ & $\begin{array}{l}\text { evaluated for anmincis } \\
\qquad 20 \% \mathrm{D} \\
\pm 25 \% \mathrm{D} \text { (liq.) \& } \pm 30 \% \mathrm{D} \text { (solid) } \\
\pm 25 \text { RPD (liq.), } \pm 30 \mathrm{RPD} \text { (solid) } \\
\text { see gross alpha/beta criteria }\end{array}$ \\
\hline Th Determination & $\begin{array}{l}\text { EPA-901.1 } \\
\text { RML-RA09 }\end{array}$ & $\begin{array}{l}\text { method blank (optional) } \\
\text { laboratory control sample } \\
\text { matrix spike } \\
\text { matrix spike duplicate or sample duplicate } \\
\text { associated instrument } Q C\end{array}$ & $\begin{array}{l}\text { none specified } \\
\text { none specified } \\
\text { none specified } \\
\text { none specified } \\
\text { none specified }\end{array}$ & $\begin{array}{l}\text { evaluated for contamination' } \\
\qquad 20 \% \mathrm{D} \\
\pm 25 \% \mathrm{D} \text { (liq.) \& } \pm 30 \% \mathrm{D} \text { (solid) } \\
\pm 25 \mathrm{RPD} \text { (liq.), } \pm 30 \mathrm{RPD} \text { (solid) } \\
\text { see gamma spectrometry criteria }\end{array}$ \\
\hline $\begin{array}{l}\text { PCBs } \\
\text { (polychlorinated- } \\
\text { biphenyls) }\end{array}$ & SW846-8080 & $\begin{array}{l}\text { calibration verification (ICV \& CCV) } \\
\text { method blank (sample prep) } \\
\text { surogate standard } \\
\text { matrix spike } \\
\text { matrix spike duplicate } \\
\text { sample duplicate } \\
\text { laboratory control sample (sample prep) }\end{array}$ & $\begin{array}{l}\text { refer to method } 8080 \\
\text { none specified } \\
\text { none specified } \\
\text { none specified } \\
\text { none specified } \\
\text { none specified } \\
\text { none specified }\end{array}$ & $\begin{array}{l}\text { to be specified } \\
\text { <regulatory limit (2ppm) } \\
\pm 50-150 \% R \\
\pm 50-150 \% R \\
\pm 50-150 \% R \\
\text { to be specified } \\
\text { to be specified }\end{array}$ \\
\hline Volatile organics & SW846-8260 & $\begin{array}{l}\text { calibration verification (ICV \& CCV)' } \\
\text { method blank (sample prep)' } \\
\text { surrogate standard } \\
\text { matrix spike } \\
\text { matrix spike duplicate } \\
\text { sample duplicate } \\
\text { laboratory control sample (sample prep) }\end{array}$ & see SW846 8260, Sept. '86 & $\begin{array}{l}\quad \pm 20 \% \mathrm{D} \\
\quad 3 \mathrm{XMDL} \\
\text { refer to supplement Table A } \\
\text { refer to supplement Table A } \\
\text { refer to supplement Table A } \\
\text { refer to supplement Table A } \\
\text { refer to supplement Table A }\end{array}$ \\
\hline $\begin{array}{l}\text { Nonhalogenated } \\
\text { volatile organics }\end{array}$ & SW846-8015 & $\begin{array}{l}\text { calibration verification (ICV \& CCV' } \\
\text { method blank (sample prep)' } \\
\text { surogate standard } \\
\text { matrix spike } \\
\text { matrix spike duplicate } \\
\text { sample duplicate } \\
\text { laboratory control sample (sample prep)" }\end{array}$ & $\begin{array}{c}\text { see SW846-8015, Sept. '86 } \\
\vdots \\
\vdots \\
\vdots\end{array}$ & $\begin{array}{l}\qquad 15 \% \mathrm{D} \\
\quad 3 \times \mathrm{MDL} \\
\text { refer to supplement Table B } \\
\text { refer to supplement Table B } \\
\text { refer to supplement Table B } \\
\text { refer to supplement Table B } \\
\text { refer to supplement Table B }\end{array}$ \\
\hline $\begin{array}{l}\text { Semivolatile } \\
\text { organics }\end{array}$ & SW846-8270 & $\begin{array}{l}\text { calibration verification (ICV \& CCV) } \\
\text { method blank (sample prep) } \\
\text { surrogate standard } \\
\text { matrix spike } \\
\text { matrix spike duplicate } \\
\text { sample duplicate } \\
\text { labcratory control sample (sample prep) }\end{array}$ & $\begin{array}{c}\text { see SW846-8270, Sept. '86 } \\
\vdots \\
\vdots \\
\vdots\end{array}$ & $\begin{array}{l}\quad \pm 20 \% \mathrm{D} \\
3 \mathrm{XMDL} \\
\text { refer to supplement Table C } \\
\text { refer to supplement Table C } \\
\text { refer to supplement Table C } \\
\text { refer to supplement Table C } \\
\text { refer to supplement Table C }\end{array}$ \\
\hline
\end{tabular}


Initial calibration verification (ICV) is typically performed at the beginning of a run to check the calibration and must be independent of the calibration standards. The continuing calibration verification (CCV) must also be independent of the calibration standards, but may be the same standard as the ICV. The CCV is typically analyzed every 10 samples and at the end of the run for metals analysis or every 12 samples for organic analysis.

b The calibration blank is an instrument blank used in the calibration to initially determine the blank value and therefore used as blank subtraction. The continuing calibration blank (CCB) is also an instrument blank which is analyzed every 10 samples and at the end of the run, but is not used in blank subtraction, but only to monitor instrument contamination.

Method blanks and laboratory control samples are only required if a sample preparation is performed before analysis. Sample preparation does not include dilutions or transfers to containers.

d Post digestion spikes are not necessary if the pre-digestion spike is in control. If this control does not meet the QC acceptance criteria, the post digestion spike should be performed.

Acceptance criteria:

$\% \mathrm{D}=\%$ deviation from true value

$\% \mathrm{R}=\%$ recovery of true value

$\mathrm{RPD}=$ relative percent difference between two compared values

f Method blanks for radiochemical analysis are used to monitor cross contamination. However, due to the levels of radioactivity present in samples at the RMAL, the effect of contamination may be insignificant in most cases. Therefore, the requirement to analyze a method blank for radiochemical analysis is optional (i.e. at the discretion of the chemist or supervisor).

g Acceptance criteria for the method blanks performed for radiochemical analysis varies based upon the level of activity in the samples and the amount of background activity. A qualified chemist reviews the data from method blanks to determine if significant contamination is present.

h The acceptance criteria for PCB analyses which are not identified in this table, shall be specified at a later date. Currently, the Analytical Methods Group group leader specifies the QC criteria if different from SW846 and if not specified by the sample generator. 
SUPPLEMENT TABLE A

Volatile Organic Analyses QC Limits

\begin{tabular}{|c|c|c|c|c|c|c|}
\hline CAS\# & Compound & $\begin{array}{c}\text { Precision } \\
\text { (RPD) }\end{array}$ & $\begin{array}{c}\text { Accuracy } \\
(\% \mathrm{R})\end{array}$ & $\begin{array}{c}\text { MDL } \\
\text { (mg/Kg) }\end{array}$ & $\begin{array}{c}\text { PRQL } \\
\text { (mg/Kg) }\end{array}$ & $\begin{array}{c}\text { LCS } \\
(\% \mathrm{R}) \\
\end{array}$ \\
\hline $75-01-4$ & Vinyl Chloride & $\leq 200$ & D-251 & 1 & 4 & $34-100$ \\
\hline $75-69-4$ & Trichlorofluoromethane & $\leq 110$ & $17-181$ & 1 & 10 & $47-103$ \\
\hline 76-13-1 & 1,1,2-Trichloro-1,2-2-Trifluoroethane & $\leq 50$ & $60-150$ & 1 & 10 & $49-105$ \\
\hline $75-35-4$ & 1,1-Dichloroethylene & $\leq 250$ & $\mathrm{D}-234$ & 1 & 10 & $43-100$ \\
\hline $75-9-2$ & Methylene Chloride & $\leq 50$ & D-221 & 1 & 10 & $67-108$ \\
\hline $75-15-0$ & Carbon Disulfide & $\leq 50$ & $60-150$ & 1 & 10 & $36-100$ \\
\hline $67-66-3$ & Chloroform & $\leq 44$ & $51-138$ & 1 & 10 & $72-111$ \\
\hline $107-6-2$ & 1,2-Dichloroethane & $\leq 42$ & $49-155$ & 1 & 10 & $76-112$ \\
\hline $71-55-6$ & 1,1,1-Trichloroethane & $\leq 33$ & $52-162$ & 1 & 10 & $71-110$ \\
\hline $56-23-5$ & Carbon Tetrachloride & $\leq 30$ & $70-140$ & 1 & 10 & $54-115$ \\
\hline $71-43-2$ & Benzene & $\leq 45$ & $37-151$ & 1 & 10 & $70-109$ \\
\hline $79-1-6$ & Trichloroethylene & $\leq 36$ & $71-157$ & 1 & 10 & $80-120$ \\
\hline $79-0-5$ & 1,1,2-Trichloroethane & $\leq 38$ & $52-150$ & 1 & 10 & $80-120$ \\
\hline $75-25-2$ & Bromoform & $\leq 47$ & $45-169$ & 1 & 10 & $61-115$ \\
\hline $108-88-3$ & Toluene & $\leq 29$ & $47-150$ & 1 & 10 & $80-120$ \\
\hline $127-18-4$ & Tetrachloroethylene & $\leq 29$ & 64-148 & 1 & 10 & $80-120$ \\
\hline $108-90-7$ & Chlorobenzene & $\leq 38$ & $37-160$ & 1 & 10 & $80-120$ \\
\hline $100-41-4$ & Ethylbenzene & $\leq 43$ & $37-162$ & 1 & 10 & $80-120$ \\
\hline $1330-20-7$ & Xylenes & $\leq 50$ & $60-150$ & 1 & 10 & $80-120$ \\
\hline $79-34-5$ & 1,1,2,2-Tetrachloroethane & $\leq 55$ & $46-157$ & 1 & 10 & $67-117$ \\
\hline $106-46-7$ & 1,4-Dichlorobenzene & $\leq 60$ & $18-190$ & 1 & 10 & $80-120$ \\
\hline $95-50-1$ & ortho-Dichlorobenzene & $\leq 60$ & $18-190$ & 1 & 10 & $80-112$ \\
\hline $60-29-7$ & Ethyl Ether & $\leq 50$ & $60-150$ & 1 & 10 & $54-100$ \\
\hline \multicolumn{7}{|c|}{ Surrogates } \\
\hline & 1,2-Dichloroethane-d $d_{4}$ & & $61-129$ & & & \\
\hline & Toluene- $d_{8}$ & & $89-118$ & & & \\
\hline & 4-Bromofluorobenzene & & $93-107$ & & & \\
\hline
\end{tabular}


SUPPLEMENT TABLE B

Nonhalogenated Volatile Organic Analyses QC Limits

\begin{tabular}{|c|c|c|c|c|c|c|}
\hline CAS\# & Compound & $\begin{array}{c}\text { Precision } \\
\text { (RPD) } \\
\end{array}$ & $\begin{array}{l}\text { Accuracy } \\
(\% \text { R) }\end{array}$ & $\begin{array}{c}\mathrm{MDL} \\
(\mathrm{mg} / \mathrm{Kg})\end{array}$ & $\begin{array}{c}\text { PRQL } \\
(\mathrm{mg} / \mathrm{Kg})\end{array}$ & $\begin{array}{c}\text { LCS } \\
(\% \mathrm{R}) \\
\end{array}$ \\
\hline $67-56-1$ & Methanol & $\leq 50$ & $60-150$ & 10 & 100 & $49-145$ \\
\hline $67-64-1$ & Acetone & $\leq 50$ & $60-150$ & 10 & 100 & $61-136$ \\
\hline $78-93-3$ & $\begin{array}{l}\text { Methyl Ethyl } \\
\text { Ketone }\end{array}$ & $\leq 50$ & $60-150$ & 10 & 100 & $62-134$ \\
\hline $78-83-1$ & Isobutanol & $\leq 50$ & $60-150$ & 10 & 100 & $52-126$ \\
\hline $71-36-3$ & Butanol & $\leq 50$ & $60-150$ & 10 & 100 & $50-110$ \\
\hline $110-86-1$ & Pyridine & $\leq 50$ & $60-150$ & 10 & 100 & $64-122$ \\
\hline \multicolumn{7}{|c|}{ 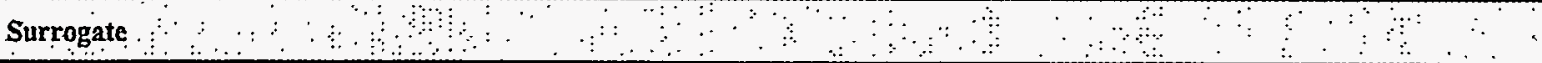 } \\
\hline $71-23-8$ & n-Propanol & & $60-150$ & & & \\
\hline
\end{tabular}

SUPPLEMENT TABLE C

Semivolatile Organic Analyses QC Limits

\begin{tabular}{|c|c|c|c|c|c|c|}
\hline$\overline{\mathrm{CAS} \#}$ & Compound & $\begin{array}{l}\text { Precision } \\
\text { (RPD) }\end{array}$ & $\begin{array}{l}\text { Accuracy } \\
(\% \mathrm{R})\end{array}$ & $\begin{array}{c}\mathrm{MDL} \\
(\mathrm{mg} / \mathrm{Kg})\end{array}$ & $\begin{array}{c}\text { PRQL } \\
(\mathrm{mg} / \mathrm{Kg})\end{array}$ & $\begin{array}{l}\text { LCS } \\
(\% \mathrm{R})\end{array}$ \\
\hline $95-48-7$ & 2-Methylphenol & $\leq 50$ & $60-150$ & 5 & 40 & $46-104$ \\
\hline $67-72-1$ & Hexachloroethane & $\leq 44$ & $40-113$ & 5 & 40 & $38-100$ \\
\hline $106-44-5$ & 4-Methylphenol & $\leq 50$ & $60-150$ & 5 & 40 & $46-114$ \\
\hline $98-95-3$ & Nitrobenzene & $\leq 72$ & $35-180$ & 5 & 40 & $46-100$ \\
\hline $121-14-2$ & 2,4-Dinitrotoluene & 546 & $39-139$ & 0.3 & 2.6 & $54-146$ \\
\hline $118-74-1$ & Hexachlorobenzene & $\leq 319$ & D-152 & 0.3 & 2.6 & $52-115$ \\
\hline $87-86-5$ & Pentachlorophenol & $\leq 128$ & $14-176$ & 5 & 40 & $54-130$ \\
\hline $51-28-5$ & 2,4-Dinitrophenol & $\leq 119$ & D-172 & 5 & 40 & $47-100$ \\
\hline \multicolumn{7}{|c|}{ 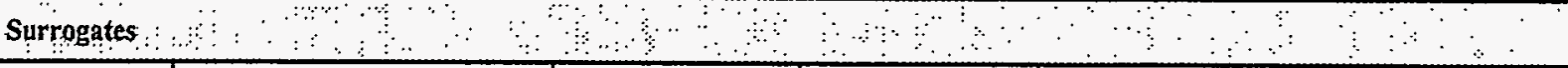 } \\
\hline \multirow[t]{3}{*}{$367-12-4$} & 2-Fluorophenol & & D-107 & & & \\
\hline & Phenol-ds & & 8-142 & & & \\
\hline & Nitrobenzene- $d_{s}$ & & $28-117$ & & & \\
\hline \multirow[t]{3}{*}{$321-60-8$} & 2-Fluorobiphenyl & & $24-144$ & & & \\
\hline & 2,4,6-Tribromophenol & & D-100 & & & \\
\hline & Terphenyl- $d_{14}$ & & D-226 & & & \\
\hline
\end{tabular}




\section{APPENDIX B}

This section includes three tables of information and measurements that may be of value to the data users. The first Table B1, includes the field measurements taken from the top of the tank to each phase change (air/liquid, liquid/sludge, and bottom of the tank). Table B1 also includes the total mass and/or activity for some of the major species in the sludge of general interest to the data users.

The dose measurement taken in during the field sampling for the liquid and sludge samples are included in Table B2 and Table B3. The dose measurements were taken at contact with the sampling container ( $250 \mathrm{~mL}$ I-Chem jar) for the liquids and at contact with the one inch core sludge sampling device. 
Table B1 Total Mass and Activity for Selected Species of Interest in Sludge

\begin{tabular}{|c|c|c|c|c|c|c|c|c|}
\hline Measturement 1 & 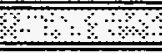 & W-24 & WV:25 & $3 W-26$ & $W=27$ & W-28 & W-31: & \\
\hline Depth to top of liquid & (in.) & $192^{\circ}$ & 140 & 139 & 184 & 140 & 148 & \\
\hline Depth to top of sludge & (in.) & 225 & 204 & 214 & 206 & 235 & 218 & \\
\hline Depth to top of hard sludg & e (in.) & 258 & 258 & 255 & 249 & 256 & 247 & \\
\hline Depth to bottom of tank & (in.) & 258 & 258 & 255 & 257 & 256 & 256 & \\
\hline Depth of supernatant & (in.) & 33 & 64 & 75 & 22 & 95 & 70 & \\
\hline Depth of soft Sludge & (in.) & 33 & 54 & 41 & 43 & 21 & 29 & \\
\hline Depth of hard Sludge & (in.) & 0 & 0 & 0 & 8 & 0 & 9 & \\
\hline Total depth of Sludge & (in.) & 33 & 54 & 41 & 51 & 21 & 38 & \\
\hline Stininaty of fank rod & messandidis & Wigernass & & & 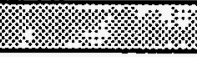 & ?. & $2 \%$ & Total \\
\hline Volume of Supernatant & $(L)$ & 51500 & 101500 & 124200 & 37900 & 150300 & 126100 & 591500 \\
\hline Volume of Sludge & $(\mathrm{L})$ & 32900 & 65500 & 44700 & 60600 & 17000 & 40100 & 260800 \\
\hline Density of Sludge & $(\mathrm{Kg} / \mathrm{L})$ & 1.37 & 1.36 & 1.38 & 1.44 & 1.37 & 1.44 & \\
\hline Mass of Sludge & $(\mathrm{Kg})$ & 45073 & 89080 & 61686 & 87264 & 23290 & 57744 & 364137 \\
\hline 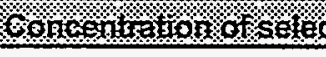 & 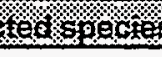 & 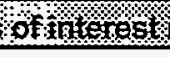 & imsintadge & 8 \% & ঋে. & י্যে:- & 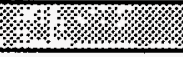 & $\sqrt{1.4 \%,: ~}$ \\
\hline Thorium & $(\mathrm{mg} / \mathrm{Kg})$ & 3270 & 9250 & 3280 & 1290 & 1360 & 20700 & \\
\hline Uranium & $(\mathrm{mg} / \mathrm{Kg})$ & 6780 & 7660 & 1.9400 & 11700 & 18500 & 19800 & \\
\hline Plutonium & $(\mathrm{mg} / \mathrm{Kg})$ & 0.96 & 1.73 & 0.70 & 0.35 & 0.45 & 1.82 & \\
\hline${ }^{233} \mathrm{U}$ & $(\mathrm{mg} / \mathrm{Kg})$ & 3.6 & 6.6 & 25.1 & 2.5 & 12.0 & 10.9 & \\
\hline${ }^{235} \mathrm{U}$. & $(\mathrm{mg} / \mathrm{Kg})$ & 33.2 & 45.2 & 51.3 & 35.7 & 46.2 & 121.0 & \\
\hline${ }^{239} \mathrm{Pu}$ & $(\mathrm{mg} / \mathrm{Kg})$ & 0.84 & 1.46 & 0.57 & 0.29 & 0.36 & 1.49 & \\
\hline r z d f & 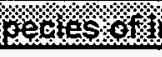 & 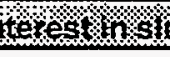 & mag & & & \% & (16? & \%ి. \\
\hline${ }^{90} \mathrm{Sr}$ & $(B q / g)$ & 1400000 & 3200000 & 710000 & 450000 & 700000 & 11000000 & \\
\hline${ }^{137} \mathrm{Cs}$ & $(\mathrm{Bq} / \mathrm{g})$ & 530000 & 470000 & 890000 & 390000 & 310000 & 430000 & \\
\hline${ }^{233} \mathrm{U}$ & $(B q / g)$ & 1600 & 2800 & 10000 & 1000 & 5200 & 5200 & \\
\hline${ }^{238} \mathrm{Pu}$ & $(B q / g)$ & 3800 & 7800 & 5400 & 2400 & 3000 & 13000 & \\
\hline${ }^{241} \mathrm{Am}$ & $(\mathrm{Bq} / \mathrm{g})$ & 3900 & 9300 & 3900 & 2800 & 4600 & 14000 & \\
\hline${ }^{244} \mathrm{Cm}$ & $(\mathrm{Bq} / \mathrm{g})$ & 22000 & 58000 & 28000 & 17000 & 25000 & 110000 & \\
\hline 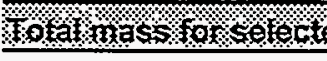 & STperias: & 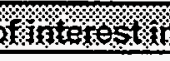 & 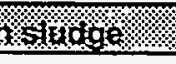 & (1) & ঙ \% & ওং\% & 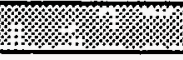 & Totat \\
\hline Thorium & $(\mathrm{Kg})$ & 147.4 & 824.0 & 202.3 & 112.6 & 31.7 & 1195.3 & 2513.3 \\
\hline Uranium & $(\mathrm{Kg})$ & 305.6 & 682.4 & 1196.7 & 1021.0 & 430.9 & 1143.3 & 4779.8 \\
\hline Plutonium & (Kg) & 0.043 & 0.154 & 0.043 & 0.030 & 0.010 & 0.105 & 0.386 \\
\hline${ }^{233} \mathrm{U}$ & $(\mathrm{Kg})$ & 0.162 & 0.588 & 1.548 & 0.220 & 0.279 & 0.629 & 3.427 \\
\hline${ }^{235} \mathrm{U}$ & $(\mathrm{Kg})$ & 1.496 & 4.026 & 3.164 & 3.115 & 1.076 & 6.987 & 19.866 \\
\hline${ }^{239} \mathrm{Pu}$ & $(\mathrm{Kg})$ & 0.038 & 0.130 & 0.035 & 0.026 & 0.008 & 0.086 & 0.323 \\
\hline 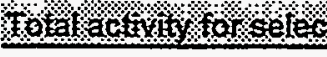 & cosspecues & 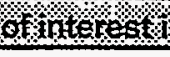 & 1/3atage. & (1. & স্যে: & & (1:- & Kotat \\
\hline${ }^{\mathrm{s}} \mathrm{Sr}$ & (Ci) & 1705.46 & 7704.22 & 1183.70 & 1061.32 & 440.62 & 17167.14 & 29262.5 \\
\hline${ }^{137} \mathrm{Cs}$ & (Ci) & 645.64 & 1131.56 & 1483.80 & 919.81 & 195.13 & 671.08 & 5047.0 \\
\hline${ }^{233} \mathrm{U}$ & (Ci) & 1.95 & 6.74 & 16.67 & 2.36 & 3.27 & 8.12 & 39.1 \\
\hline${ }^{238} \mathrm{Pu}$ & (Ci) & 4.63 & 18.78 & 9.00 & 5.66 & 1.89 & 20.29 & 60.2 \\
\hline${ }^{241} \mathrm{Am}$ & (Ci) & 4.75 & 22.39 & 6.50 & 6.60 & 2.90 & 21.85 & 65.0 \\
\hline${ }^{244} \mathrm{Cm}$ & (Ci) & 26.80 & 139.64 & 46.68 & 40.09 & 15.74 & 171.67 & 440.6 \\
\hline
\end{tabular}

